\title{
A graph-based approach for online multi-object tracking in structured videos with an application to action recognition
}

\author{
Henrique Morimitsu
}

THESIS PRESENTED

TO THE

Institute of Mathematics And Statistics

OF THE

University of SÃo PAUlo

FOR

OBTAINING THE TITLE

$\mathrm{OF}$

Ph.D. In SCIENCES

\author{
Program: Computer Science \\ Advisor: Prof. Dr. Roberto Marcondes Cesar Junior \\ The author received financial support from \\ FAPESP (grants \#2012/09741-0 and \#2013/08258-7) and CAPES.
}

São Paulo, December 2015 


\section{A graph-based approach for online multi-object tracking in structured videos with an application to action recognition}

This version of the thesis contains the corrections and suggestions given by the Judging Committee during the defense of the original version of the work, that took place on October 20th, 2015. A copy of the original version is available at the Institute of Mathematics and Statistics of the University of São Paulo.

Judging Committee:

- Prof. Dr. Roberto Marcondes Cesar Junior (advisor) - IME-USP

- Prof. Dr. Isabelle Bloch - Télécom ParisTech

- Prof. Dr. Junior Barrera - IME-USP

- Prof. Dr. Anderson de Rezende Rocha - UNICAMP

- Prof. Dr. William Robson Schwartz - UFMG 


\section{Acknowledgements}

I would like to thank my advisor, Prof. Roberto Marcondes Cesar Junior. This work could not have been completed without him. Thank you for your trust, dedication and guidance during all this time. I would also like to give special thanks to Prof. Isabelle Bloch who not only welcomed me during one year at Télécom ParisTech, but also greatly contributed to the conclusion of this project from the beginning until the very end. Her knowledge was invaluable to the development of this thesis as well as my own.

I thank my family, who has been supporting me all the way, sharing all the good moments together and helping me to overcome the hardships along the way. I also thank all the friends I made during this journey, who contributed to this work directly or indirectly. Special thanks to those that I had more contact with: Prof. Roberto Hirata, Eric Keiji, Estephan Wandekoken, Frank Aguilar, Guillaume Tartavel, Qin Qiao, Silvia Cristina and Yuke Li.

I offer my thanks to São Paulo Research Foundation - FAPESP (grants \#2011/507612, \#2012/09741-0 and \#2013/08258-7), CNPq, CAPES and NAP eScience - PRP - USP for providing financial support to conclude this work. I also thank the Institute of Mathematics and Statistics for receiving me and providing me all the necessary structure for the development of the thesis. 


\section{Resumo}

MORIMITSU, H. Uma abordagem baseada em grafos para rastreamento de múltiplos objetos em vídeos estruturados com uma aplicação para o reconhecimento de ações. 2015. 86 f. Tese (Doutorado) - Instituto de Matemática e Estatística, Universidade de São Paulo, São Paulo, 2015.

Nesta tese, uma nova abordagem para o rastreamento de múltiplos objetos com o uso de informação estrutural é proposta. Os objetos são rastreados usando uma combinação de filtro de partículas com descrição das imagens por meio de Grafos Relacionais com Atributos (ARGs). O processo é iniciado a partir do aprendizado de um modelo de grafo estrutural probabilístico utilizando imagens anotadas. Os grafos são usados para avaliar o estado atual do rastreamento e corrigi-lo, se necessário. Desta forma, o método proposto é capaz de lidar com situações desafiadoras como movimento abrupto e perda de rastreamento devido à oclusão. A principal contribuição desta tese é a exploração do modelo estrutural aprendido. Por meio dele, a própria informação estrutural da cena é usada para guiar o processo de deteç̧ão em caso de perda do objeto. Tal abordagem difere de trabalhos anteriores, que utilizam informação estrutural apenas para avaliar o estado da cena, mas não a consideram para gerar novas hipóteses de rastreamento. A abordagem proposta é bastante flexível e pode ser aplicada em qualquer situação em que seja possível encontrar padrões de relações estruturais entre os objetos.

O rastreamento de objetos pode ser utilizado para diversas aplicações práticas, tais como vigilância, análise de atividades ou navegação autônoma. Nesta tese, ele é explorado para rastrear diversos objetos em vídeos de esporte, na qual as regras do jogo criam alguns padrões estruturais entre os objetos. Além de detectar os objetos, os resultados de rastreamento também são usados como entrada para reconhecer a ação que cada jogador está realizando. Esta etapa é executada classificando um segmento da sequência de rastreamento por meio de Modelos Ocultos de Markov (HMMs). A abordagem de rastreamento proposta é testada em diversos vídeos de jogos de tênis de mesa e na base de dados ACASVA, demonstrando a capacidade do método de lidar com situações de oclusão ou cortes de câmera.

Palavras-chave: Rastreamento de objetos, grafo, informação estrutural, filtro de partículas, reconhecimento de ações. 


\section{Abstract}

H. MORIMITSU. A graph-based approach for online multi-object tracking in structured videos with an application to action recognition. 2015. 86 p. Thesis (Ph.D.) - Institute of Mathematics and Statistics, University of São Paulo, São Paulo, 2015.

In this thesis we propose a novel approach for tracking multiple objects using structural information. The objects are tracked by combining particle filter and frame description with Attributed Relational Graphs (ARGs). We start by learning a structural probabilistic model graph from annotated images. The graphs are then used to evaluate the current tracking state and to correct it, if necessary. By doing so, the proposed method is able to deal with challenging situations such as abrupt motion and tracking loss due to occlusion. The main contribution of this thesis is the exploration of the learned probabilistic structural model. By using it, the structural information of the scene itself is used to guide the object detection process in case of tracking loss. This approach differs from previous works, that use structural information only to evaluate the scene, but do not consider it to generate new tracking hypotheses. The proposed approach is very flexible and it can be applied to any situation in which it is possible to find structural relation patterns between the objects.

Object tracking may be used in many practical applications, such as surveillance, activity analysis or autonomous navigation. In this thesis, we explore it to track multiple objects in sports videos, where the rules of the game create some structural patterns between the objects. Besides detecting the objects, the tracking results are also used as an input for recognizing the action each player is performing. This step is performed by classifying a segment of the tracking sequence using Hidden Markov Models (HMMs). The proposed tracking method is tested on several videos of table tennis matches and on the ACASVA dataset, showing that the method is able to continue tracking the objects even after occlusion or when there is a camera cut.

Keywords: Object tracking, graph, structural information, particle filter, action recognition. 


\section{Contents}

$\begin{array}{ll}\text { List of abbreviations } & \text { ix }\end{array}$

List of symbols $\quad$ xi

List of figures $\quad$ xiii

List of tables $\quad$ XV

1 Introduction $\quad 1$

1.1 Motivation . . . . . . . . . . . . . . . . . 2

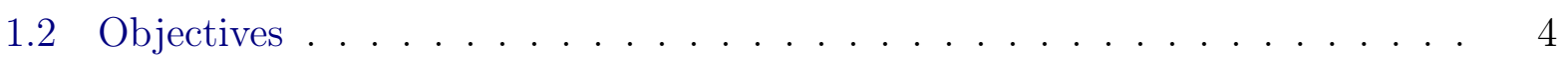

1.3 Contributions . . . . . . . . . . . . . . . . . . . 4

1.4 Organization of this thesis . . . . . . . . . . . . . . 5

$\begin{array}{lll}2 & \text { Related work } & 7\end{array}$

2.1 Multi-object tracking . . . . . . . . . . . . . . . . . . . 7

2.2 Action recognition . . . . . . . . . . . . . . . . . 11

3 Multi-object tracking based on structural information 13

3.1 Framework . . . . . . . . . . . . . . . . . . . . 13

3.2 Tracking objects with particle filters . . . . . . . . . . . . . . . . 15

3.2.1 Bayes recursive filter . . . . . . . . . . . . . . . 15

3.2 .2 Particle filter . . . . . . . . . . . . . . . . . . 18

3.2.3 State and dynamics models . . . . . . . . . . . . . . . . . 19

3.2.4 Color histogram-based tracking . . . . . . . . . . . . . . . 19

3.3 Building the graphs . . . . . . . . . . . . . . . . . . 20

3.3.1 Attributed Relational Graph (ARG) . . . . . . . . . . . . . . . 20

3.3.2 Model graph . . . . . . . . . . . . . . . . . . . . . . . 21

3.3.3 Scene graph . . . . . . . . . . . . . . . . . . 22

3.4 Tracking using graphs . . . . . . . . . . . . . . . . . . . . 23

3.4.1 Generating new candidates . . . . . . . . . . . . . . 23

3.4.2 Computing temporal scores of trackers . . . . . . . . . . . . . 24

3.4.3 Generating scene graphs for evaluation . . . . . . . . . . . 25 
3.4.4 Removing undesired trackers . . . . . . . . . . . . . . . . 27

3.4.5 Choosing the final trackers . . . . . . . . . . . . . . . 28

4 Action recognition $\quad 31$

4.1 Histogram of Oriented Gradients (HOG) . . . . . . . . . . . . . . . . 32

4.2 Principal Components Analysis (PCA) . . . . . . . . . . . . . . . 33

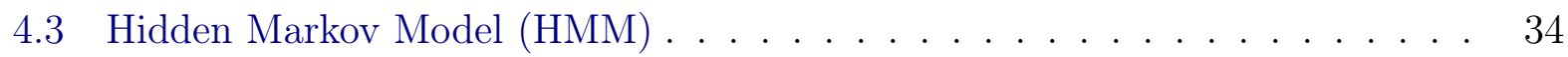

4.3.1 Evaluation . . . . . . . . . . . . . . . . . . 37

4.3 .2 Classification . . . . . . . . . . . . . . . . . . . . 39

4.3 .3 Learning . . . . . . . . . . . . . . . . . . . . 40

$\begin{array}{lll}5 & \text { Results } & 47\end{array}$

5.1 Multi-object tracking . . . . . . . . . . . . . . . . . 47

5.1 .1 Datasets ........................... 47

5.1 .2 Evaluation measurements . . . . . . . . . . . . . . 48

5.1 .3 Estimation of parameters . . . . . . . . . . . . . . . 50

5.1 .4 Results on datasets . . . . . . . . . . . . . . . . . . 51

5.2 Action recognition . . . . . . . . . . . . . . . . . 55

5.2 .1 Dataset . . . . . . . . . . . . . . . . 56

5.2 .2 Estimation of parameters . . . . . . . . . . . . . . 57

5.2 .3 Results on dataset . . . . . . . . . . . . . . . . . . . 58

$\begin{array}{lll}6 & \text { Conclusions } & 61\end{array}$

6.1 Contributions and discussion . . . . . . . . . . . . . . . . 61

6.2 Limitations and Future work . . . . . . . . . . . . . . . . . 62

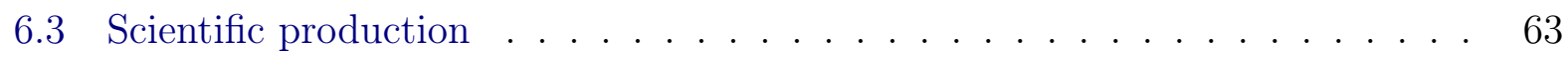

$\begin{array}{ll}\text { Bibliography } & 65\end{array}$ 


\title{
List of abbreviations
}

\author{
ARG Attributed Relational Graphs \\ CDIS Center displacement measurement \\ CERR Center error measurement \\ GINT Global intersection measurement \\ HITR Hit ratio measurement \\ HITT Hit team ratio measurement \\ HOG Histogram of Oriented Gradients \\ HMM Hidden Markov Model \\ HSV Hue - Saturation - Value color space \\ OBJI Object intersection measurement \\ PCA Principal Components Analysis \\ PDF Probability Density Function \\ SVD Singular Value Decomposition
}


$\mathrm{x}$ LIST OF ABBREVIATIONS 


\section{List of symbols}

Unless stated otherwise, the following symbol convention will be followed along the text:

- Bold lowercase: represents vectors or sequences.

- Bold uppercase: represents matrices.

- Calligraphic: represents sets.

$\alpha \quad$ HMM forward variable

$\beta \quad$ HMM backward variable

$\delta \quad$ Dirac delta function

$\gamma \quad$ HMM variable representing the probability of being at a given state or transition

$\zeta \quad$ Particle cloud confidence score

$\eta \quad$ Particle weight

$\theta \quad$ Edge angle function

$\mu \quad$ Mean or average

$\xi \quad$ HMM variable representing the probability of using one mixture

$\boldsymbol{\pi}$ Vector of HMM starting probabilities

$\rho_{F} \quad$ Feature weight constant

$\rho_{T} \quad$ Temporal weight constant

$\sigma \quad$ Standard deviation

$\tau_{d_{D}} \quad$ Different object distance threshold

$\tau_{d_{S}} \quad$ Same object distance threshold

$\tau_{O} \quad$ Overlapping threshold

$\tau_{S} \quad$ Score threshold

$\tau_{T} \quad$ Threshold for changing trackers

$\phi \quad$ Appearance score function

$\psi \quad$ Structural score function 
$\boldsymbol{A}$ Matrix of HMM state transitions

$\mathcal{A}_{\mathcal{E}} \quad$ Set of edge attributes

$\mathcal{A}_{\mathcal{V}} \quad$ Set of vertex attributes

$\boldsymbol{b}$ Vector of HMM emission probabilities

C Covariance matrix

d Distance function

e Graph edge

$f \quad$ Graph instantaneous score function

$\mathcal{E} \quad$ Set of graph edges

$\mathcal{F} \quad$ Set of final trackers

$G^{M} \quad$ Model graph

$G^{S} \quad$ Scene graph

$H \quad$ Histogram

I Digital image function

I Identity matrix

$\boldsymbol{K} \quad$ Kernel matrix

$\boldsymbol{M}_{\boldsymbol{A}}$ Matrix of graph adjacencies

$M_{C} \quad$ Matrix of target candidates generation

$N_{C} \quad$ Number of columns

$N_{F} \quad$ Number of frames

$N_{M} \quad$ Number of mixture models

$N_{O} \quad$ Number of objects

$N_{P} \quad$ Number of particles

$N_{R} \quad$ Number of rows

$N_{S} \quad$ Number of states

$N_{W} \quad$ Length of the sliding window

$\mathcal{N} \quad$ Normal or Gaussian distribution function

$o \quad$ One observation

$\boldsymbol{o} \quad$ One sequence of observations

$O$ Random variable representing one observation

$P \quad$ One tracker

$\mathcal{P} \quad$ Set of trackers

P Probability

$r \quad$ Particle consensus function

$s \quad$ One HMM state

$S$ Random variable representing one HMM state

$\mathcal{S} \quad$ Set of HMM states

$T \quad$ Total time length

$v \quad$ Graph vertex

$\mathcal{V} \quad$ Set of graph vertices

$w \quad$ Tracker confidence score

Z HMM 


\section{List of figures}

1.1 Example of a multi-object tracking situation. Most single object trackers are able to successfully track the targets when their appearance is clear (a). However, when overlap occurs (b), they are not able to solve the ambiguity problem in appearance and the tracking is lost (c). We propose to recover tracking after such events by using structural spatial information encoded in graphs (d). 3

3.1 Overview of the proposed framework. . . . . . . . . . . . . . . . . 14

3.2 An example of a scene graph. . . . . . . . . . . . . . . . . . 20

3.3 The structural attributes of the edges. . . . . . . . . . . . . . . . 21

3.4 An example of a model graph with the learned attributes. The red histograms represent the vertices attributes (color model), while the green ones represent the angles and the blue ones represent the distances. . . . . . . . . . . .

3.5 Example of candidates generated for one scene. Rectangles of the same color indicate that they belong to the same object. . . . . . . . . . . . . . . . . 24

3.6 Examples of candidate graphs to be analyzed. . . . . . . . . . . . 26

3.7 Representation of all possible graphs that can be generated from the candidates. 26

4.1 Overview of the action recognition framework. . . . . . . . . . . . . 31

4.2 Overview of HOG chain. Adapted from (Dalal and Triggs (2005)). . . . . . 32

4.3 Example of a Markov model with three states. Each circle represents one state, and the directed edges the transitions from one state to another . . . .

4.4 Example of a Hidden Markov model. The three hidden states (inside the ellipse) represent the unobservable properties of the data. Each hidden state has a probability of emitting a certain observable signal (red arrows). . . . . 37

5.1 Sample frames from the Youtube table tennis dataset. . . . . . . . . . . . . 48

5.2 Sample frames from the ACASVA badminton dataset. . . . . . . . . . . . . 48

5.3 Center error according to feature weight $\rho_{F} \ldots \ldots \ldots \ldots 1$

5.4 (a) Center error according to old weight factor $\rho_{T}$. (b) Variation in center stability caused by changing the parameter. . . . . . . . . . . . . . 51

5.5 (a) Center error according to threshold for removing trackers $\tau_{S}$. (b) Variation in performance (frames per second) caused by changing the parameter. . . . 
5.6 Tracking results for both datasets. Each method is represented by a different color. Blue: ours, red: particle filter, green: SPOT, white: STRUCK. The first two rows show results after a camera cut, while the last ones show situations of momentary occlusion. The first column represents the initial situation, in the second column, camera cut or occlusion happens and then, the third one shows the results after a few frames. . . . . . . . . . . . . . . . 54

5.7 Center error and hit team ratio for one video from each dataset including object overlapping and camera cuts. The charts were smoothed using a moving average window of 100 frames. . . . . . . . . . . . . . . . . . 55

5.8 Sample frames from the dataset used for action recognition. . . . . . . . . . 56

5.9 Variation of classification performance according to the numbers of PCs. . . 57

5.10 Examples of action sequences, the first five columns represent the action for player 1, while the others for player 2. Each row correspond to one action: (1) others, (2) forehand, (3) backhand and (4) serve. . . . . . . . . . . . . . 59 


\section{List of tables}

5.1 Initial fixed parameters used for estimating the new ones. . . . . . . . . . 50

5.2 Parameters for the tracking framework. . . . . . . . . . . . . . 52

5.3 Observed results on both datasets. The arrows indicate whether lower or higher values are better. . . . . . . . . . . . . . . 5 53

5.4 Classification hit ratio according to the sequence length and the number of hidden states. . . . . . . . . . . . . . . . . . . . . . . 58

5.5 Final parameters for the action recognition. . . . . . . . . . . 58

5.6 Observed hit ratio results for the action recognition task . . . . . . . . 58

5.7 Confusion matrix of the action classification. . . . . . . . . . . . . 59 
xvi LIST OF TABLES 


\section{Chapter 1}

\section{Introduction}

In this thesis, the problem of tracking multiple objects in videos is explored. Tracking an object consists in finding its spatial position at every frame along the whole video. As opposed to object detection, tracking relies on some temporal properties of videos in order to restrict the search space and increase the processing speed. Particularly, smooth movement is usually a strong assumption of most trackers, i.e. the position of an object in the next frame should be very close to the position in the previous frame. However, that assumption does not always hold, as the position may change abruptly in some situations, e.g. if the video contains views from several camera viewpoints.

If the object is subject to abrupt motion, trackers relying on smooth movement will not be able to find the object again, unless it comes close to the position where the tracking was previously lost. Another situation which may cause tracking loss is when there is an ambiguity between the object descriptor and the background or another object. In this case it is possible that the tracker starts to follow the wrong target, thus causing the drift problem. Drift is one of the main problems found when tracking objects over a long period of time. In order to avoid it, it is important to have some mechanism to find the object again once it is lost. Otherwise tracking may be lost forever, which is highly undesirable. In this work we propose to redetect the object by relying on structural properties.

Structure is a very general term and may be defined differently according to the problem. For the purposes of this thesis, structure will be considered as the spatial relationships between pairs of objects that can be measured numerically (e.g. the distance between them). The structure will be explored by learning some statistical properties from these measurements and then enforcing them on the image at each frame. The topology of the objects and their relationships will be stored in graphs (more details are presented in Chapter 3). 


\subsection{Motivation}

Tracking multiple objects simultaneously is a challenging task. Due to the good results demonstrated by single object trackers, using a set of them to track several objects in the same scene could be an interesting option. However, applying this direct approach usually presents some difficulties which are not always found on the single object tracking problem.

Most of the current state-of-the-art single object trackers rely on visual appearance features to describe the object of interest, using texture and shape, among others. These approaches have two main drawbacks. The first one is that, if several objects have a similar appearance, then one or more of the trackers can lose their targets. In fact, even when dealing with only one object, tracking can be lost due to ambiguity with the background. The second, and the main problem when tracking multiple objects, is dealing with occlusion. When an object is partially of fully occluded, its appearance may change significantly, which greatly affects the tracking performance. Therefore, a good method for multi-object tracking must be able to recover tracking after it is lost due to occlusion.

In order to deal with such situations, it is necessary to: (1) realize that a tracking error occurred, and (2) recover the correct target. For this, more data should be extracted from the scene and then used to obtain the required information for making the decision. As it will be further discussed in Chapter 2, many options have been explored before, each one being most suited for a different situation. In this thesis we will be interested in studying the use of spatial relations between objects to recover or correct online tracking. Online tracking, as opposed to batch methods, only uses past information to predict the next state, and thus, can be used in real time. We argue that, in some kinds of videos where the scene represents a situation that usually follows a common spatial pattern, it is possible to choose the most likely configuration of the objects by learning some structural properties beforehand. Figure 1.1 shows an example of a table tennis video with a situation where tracking is lost after two players intersect each other. Although the interaction is brief, this already causes one of the trackers to misjudge its correct target and start to track the other player instead. We intend to solve this kind of problem by exploring the spatial properties of the scene, such as the distance between two objects.

We shall refer to videos that present discernible spatial patterns as structured videos. It is assumed that scenes (or frames) of these videos contain elements that provide a kind of stable spatial structure to be explored. A good example is sport videos. Sports rely on a set of rules that usually constrain the involved objects to follow a set of patterns. These patterns often present some spatial relationships that may be explored. For example, the rules may enforce that the objects must be restricted to a certain area, or that they always keep a certain distance among them. Another case is when evaluating videos of a set of known interactions. In this situation, if a person is always using some object, then there is 


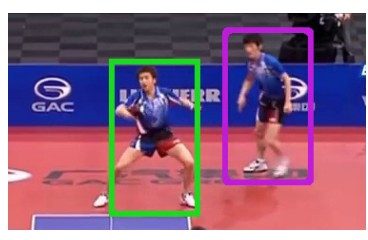

(a)

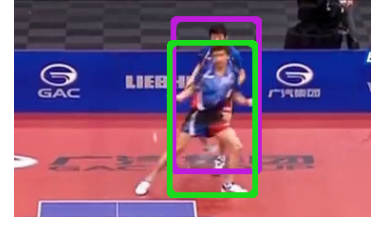

(b)

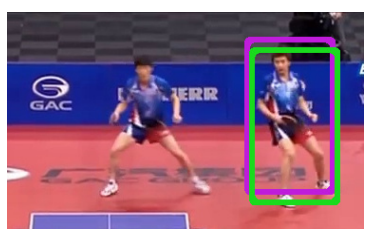

(c)

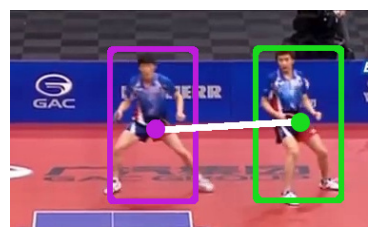

(d)

Figure 1.1: Example of a multi-object tracking situation. Most single object trackers are able to successfully track the targets when their appearance is clear (a). However, when overlap occurs (b), they are not able to solve the ambiguity problem in appearance and the tracking is lost (c). We propose to recover tracking after such events by using structural spatial information encoded in graphs (d).

usually a strong constraint on the distance and relative position between them. Structure may also be found in surveillance, where usually the location is known and controlled, thus the region of interest is constrained by the environment itself. Examples include vision-based monitoring for smart homes since people activities are stable given the environment (e.g. cooking, eating, watching TV, etc.).

Structural relations are explored by using graphs to encode the spatial configuration of the scene. In this thesis, particle filter was chosen as the single object tracker, due to its simplicity and good results demonstrated in previous studies. However, theoretically any other tracker could be used instead to benefit from the added structural information. This makes the proposed framework very flexible and able to be used to potentially improve the results of any single object tracking applied in multi-objects problems. As shall be explained throughout this text, the graphs allow us to numerically evaluate the tracking state at each frame. This value is then used to decide whether the current tracking is correct or if some object was lost and, in this case, the state must be corrected. The graphs also contain the necessary information for recovering the correct position of the target. With this approach it is possible to improve the overall result by recovering tracking after situations of overlapping between objects with similar appearance or when abrupt motion happens.

The use of structural information for recovering tracking is a topic that was not much explored before. Indeed, several of the current state of the art methods based on tracking by detection do use structural information at a different level, for evaluating the tracking state and solving the data association problem between the frames. However, the detections are usually carried out by off-the-shelf detectors that do not consider scene information. In that sense, one main contribution of this thesis is to explore the learned probabilistic structural model to guide the detection in case of tracking loss. This approach may greatly improve the performance by reducing the search space.

We also show a practical application of tracking, where the results obtained from the tracking step are used to perform action recognition. This is done by accumulating all the 
tracking results for each object over time to create sequences. Short segments of these sequences are then fed to multiple Hidden Markov Models which classify each of them into one of a given set of possible actions for each object.

The proposed framework is tested using videos obtained from Youtube and also from the ACASVA (De Campos et al. (2011)) dataset, which present more challenging conditions such as changing of lighting, perspective and camera cuts.

\subsection{Objectives}

The objective of this thesis is to propose a novel approach to improve tracking of multiple objects using structural information encoded in graphs. Besides, we also intend to show that the tracking results can be applied for performing individual action recognition for each object.

\subsection{Contributions}

The main contributions of this thesis are the following:

- a novel formalization of tracking in a flexible structural graph framework: the problem of tracking multiple objects is modeled as a structural graph optimization problem. Thus, it can be solved by relying on the structural properties extracted from the images. Besides, the formulation is flexible and may be adapted to any kind of problem where a structural graph can be defined;

- development of a probabilistic graph model that can be used to evaluate the structure of a scene: several works use object models to perform tracking by detection, but learning a structural model does not seem to have been much explored before;

- proposal of a method for dealing with abrupt motion by generating candidate object locations based on the model: most of the works rely on visual features to find the object after it is lost, but they fail to explore the structural relations;

- implementation of a novel framework for tracking multiple objects by evaluating multiple hypotheses with scene graphs: the probabilistic structural model is used to both generate new tracking hypotheses and choosing the best configuration for each frame. A framework containing the proposed model is implemented and tested on several videos to analyze its behavior in real situations. 


\subsection{Organization of this thesis}

In Chapter 2 we present a review of some relevant previous works and how they contributed to the development of our method.

In Chapter 3 the complete framework for tracking multiple objects using graphs is explained. We start by detailing the regular particle filter tracking approach and then explain how graphs are integrated in order to deal with multiple objects.

In Chapter 4 we present how the action recognition is performed. In the beginning, the overall framework is explained, followed by a detailed description of the models and methods used in this step.

In Chapter 5 the experimental results obtained are exposed. We start by showing results obtained in the tracking step. We compare the obtained results of our approach with other baseline methods. Afterwards, some results regarding the action recognition are also presented.

In Chapter 6 we discuss the main conclusions of this work as well as suggestions for future research and some final remarks. 


\section{Chapter 2}

\section{Related work}

\section{$2.1 \quad$ Multi-object tracking}

Multi-object tracking has been receiving great attention recently and many approaches have been proposed. In this chapter we will discuss about some current works to give an overview of the state of the art and then present how our approach is inserted in this field.

Predictive, or recursive models, such as the Kalman filter, have been used since the beginning to deal with multi-object tracking (Reid (1979)). More recently, particle filter has become more popular due to its ability to deal with non-linear and non-Gaussian models, which frequently appear in tracking situations.

One strategy when using particle filters is to model all the objects simultaneously as a single state and use a single filter to track all of them. However, by doing so, the state space becomes very large and a huge number of particles may be necessary to obtain good samples for estimation. Widynski et al. (2012) circumvent this problem by proposing a ranked partitioned sampling scheme for resampling the particles. This is done by iteratively updating the state vector of the particles for one object at a time. By doing so, new particles do not need to be generated from a joint distribution considering all the parameters at the same time, which in turn creates new particles that are closer to each of the objects. The main drawback is that objects that are processed later are prone to be affected by errors created when updating the state for previous ones. In that sense, the proposed ranking scheme shows its importance by providing a probabilistic score that is used to process objects that are more likely to be tracked correctly first, and thus to decrease the error for the next stages.

Another option is to track each object with a different filter. However, by itself, this approach is not able to deal with objects with similar appearance, as the particles for different objects may end up grouping in only one of them. Okuma et al. (2004) deal with this problem by using a mixture particle filter. This method joins the different filters in a single mixture model in order to represent the multimodal distribution observed when tracking multiple objects. The joint multimodal distribution assures that all the filters do not end up 
representing the same single mode and keep tracking all the objects. Hu et al. (2015) also use individual filters for each object, but they tackle the multi-object problem in another way. They propose a more robust single object tracker that uses multiple features (color, edge and Gabor textures) and sparse representation for the appearance model (Mei and Ling (2011)). In a sparse representation framework, the object is represented by a linear combination of a set of object templates and trivial templates. Object templates are obtained from successful detections in previous frames and encode visual features. Trivial templates, on the other hand, are typically a set of $w \times h$ binary vectors having only the $i$-th value set to one, where $w$ and $h$ are the width and height of the tracked image, respectively. These templates form the basis of the sparse representation and are important to both reduce the comparison overload (by using sparse templates) and deal with partial occlusion (by only considering the visible templates). The authors extend the classical approach to multi-object tracking by measuring the amount of occlusion (when overlapping occurs) and handling it accordingly. If the overlap is low, then the respective templates are used to guide the tracking of each overlapped object. However, when it is high, they ignore the visual features altogether and instead rely only on the motion model of the targets to continue tracking. Our method also uses particle filter for tracking each object individually. We explore the graph information to avoid that all trackers follow a single object. This is done by explicitly penalizing configurations where two or more trackers overlap, and trying to find a better configuration which spreads them on the scene.

Occlusion is one of the main challenges involved in multi-object tracking and some recent works focus on dealing specifically with such situations. Tang et al. (2014) propose to extend the well known Deformable Parts Model (DPM), proposed by Felzenszwalb et al. (2010), to detect both single and pairs of persons. Although the parts model is somewhat robust to partial occlusion, the authors show that the method already starts to fail in situations with more than $20 \%$ of occlusion. They demonstrate that their approach can successfully deal with different degrees of occlusion, even very severe ones. The single and pair detectors can also be combined to deal with more crowded scenes. The main drawback is that it is specifically designed for tracking two persons, and a different detector must be trained for other types of objects. Grabner et al. (2010) tackle occlusion by embedding the environment context into the model. For this, they detect a set of Harris points (Harris and Stephens (1988)) and analyze their motion over time. The points that present correlated motion with the target are used as supports to cast a vote for the object position at each time. Therefore, when occlusion happens, the object can still be tracked by relying on the supports. Our work follows a similar approach, by using the structural model to keep track or find the most likely position of the target by using the other objects as supports.

Recently, the most popular approach is the one based on tracking by detection. This method relies on using object detectors on every frame to extract target candidates. The re- 
sults obtained in this stage are usually noisy (containing both missing and wrong detections). These results are usually first grouped into temporal segments called tracklets. Tracklets are usually short segments created by connecting detections that present high appearance and position correlation between frames. The main challenge comes from trying to create longer paths from the tracklets. This corresponds to trying to solve a data correlation problem that must be robust to occlusion, appearance change and appearance of new objects. This has been one of the most active research topic on multiple object tracking, and countless methods have been proposed. We will discuss only a few recent works that represent different popular strategies.

One common choice is to model the situation as an energy minimization problem. In this setup, a cost (energy) function is defined to provide a score for each possible configuration and the state with the lowest cost should be the optimal tracking result. Milan et al. (2014) propose an energy model that is composed of six terms which consider: the distance between the estimated location and the detection, the plausibility of the motion model between frames, the distances between multiple objects (to check if occlusion happens), the trajectory persistence (continuity from one border of the image until the other), and a regularizer that constraints the number of objects. All the terms are continuous and easily differentiable, making it possible to use a gradient descent approach for minimizing the energy.

Since most multi-object tracking situations deal with human tracking, another notorious approach consists in adapting the tracking paths according to human interactions. This is usually done by modeling attraction and repulsion forces (e.g. groups tend to walk together, while two persons in opposing directions will change their paths to avoid collision). Yan et al. (2014) follow this approach by modeling three kinds of interactions: attraction, repulsion and non-interaction. They are incorporated into a Bayesian framework to update the motion model according to the interaction between each person and those that are in a surrounding area.

Methods that rely on graph representations such as Markov Random Fields (MRF) and Conditional Random Fields (CRF) have been receiving great attention lately. Zhang et al. (2015) formalize tracking as an iterative labeling problem. To this aim, the detections are considered as vertices of a graph and are connected both spatially (objects of the same frame) and temporally (between frames). Following the MRF framework, unary and binary terms are defined to evaluate the likelihood of each individual label, as well as their relations with their neighbors. The unary function is defined as the probability $\mathbb{P}(y \mid l)$ that a given object $y$ is labeled with the object ID $l$. This value is obtained both from the motion model and from a classifier that is trained with instances obtained from previous frames. The binary term is decomposed into two types: spatial and temporal. The spatial term penalizes configurations where neighbor objects receive the same label. The temporal term, on the other hand, encourages neighbor labeling if the objects have similar properties. Wen et al. 
(2014) use hierarchical hypergraphs to build higher order relations to connect the tracklets. The video is divided into segments which are iteratively connected while moving up in the hierarchy, until obtaining a unique segment for the whole track. Inside each segment, a hypergraph is built whose vertices are the tracklets and the hyperedges connect two or more vertices that are likely to be part of the same path. The attributes of the hyperedges indicate the probability of their vertices being part of the same path. The probability is computed by considering three features: appearance similarity, motion model and trajectory smoothness.

Some previous works explored structural relations to improve tracking. Perhaps the closest work to ours is that of Zhang and van der Maaten (2014). They use a model-free approach that learns the appearance and structural relations between the tracked objects online. In the first frame, manual annotations are provided and used to initially train a Histogram of Oriented Gradients (HOG) detector (Dalal and Triggs (2005)) for detecting the object in the next frames, i.e. their approach is also based on tracking by detection. The structural relations are also learned from the first frame by training a Structured Support Vector Machine (SVM), in a very similar fashion as that of the DPM detector (Felzenszwalb et al. (2010)). The models are then updated while the tracking is being performed, using a gradient descent approach. The candidate graphs are evaluated using the information obtained from the HOG detectors as well as the distances between any two objects.

Although similar, their work differs from this one in the following aspects: (1) their structural model only computes the difference between the observed distance and an ideal value that comes from the online training. Our model considers both distance and angle information obtained from a probability density function computed by evaluating several training examples. (2) Although they use the structure to improve tracking and to deal with occlusion, it is not used to guide the detection process, which could lead to improved performance by restricting the search space. Our approach uses the structural model to obtain candidates of where the target is likely to be found after tracking loss. (3) Their method of tracking by detection does not consider motion models, while we rely on particle filters.

Multi-object tracking has been applied to sports videos, with methods based on particle filters being popular (Kristan et al. (2009); Okuma et al. (2004); Xing et al. (2011)). Many works rely on background subtraction for tracking. Figueroa et al. (2006) first recover a model of the background by using morphological operations coupled with heuristics based on the intensity of the pixel values. Afterwards, the moving players are segmented by computing the difference between the frame and the obtained model. In sports videos, often multiple camera views are available. Morais et al. (2014) detect the players of a futsal game using the Viola and Jones (2004) detector and then combine the results by projecting all of them in a common projection of the field. The uncertainty of each detection is modeled by a Gaussian Mixture Model which is then used to estimate the real position of each player. 
Other authors have tried to improve tracking by exploring more complex motion models. Liu et al. (2013) used game context features designed specifically to model the motion behavior of players during a team match. Those features consider local and global properties of the scene such as relative occupancy or whether one player is chasing another. By this way, the authors design a data association function that is more suited for tracking players in team matches. Another challenging condition frequently found in sport scenes is mutual occlusion. Zhang et al. (2013) tackle this issue by using a structured sparse model for each person. This approach builds on the robustness of sparse models by reasoning that the occlusion model is usually not sparse, but rather a structured connected area. This allows the use of better models which are able to ignore features from large occlusion areas, e.g. one player occluding another.

Another important issue that must be dealt with during tracking is abrupt motion. Perhaps, the simplest way to deal with it is by generating some additional target location hypotheses around the previous location to try to cover a broader region, as applied in the work of Zhang et al. (2012). Another proposal explored by Kwon and Lee (2008) and Zhou and $\mathrm{Lu}$ (2010) is to solve the same problem using spatial position information for finding better candidates. This is done by dividing the image into several smaller regions and using the information obtained from the density of states of each one to find new locations. More recently, Su et al. (2014) propose to rely on visual saliency information to guide the tracking and restore the target. We chose to use a different approach, instead of relying on the features from the object to restore the tracking, we use the structural relations between them to find the most likely new locations of the lost object. By doing so, the lost object can be recovered as long as some are successfully tracked.

\subsection{Action recognition}

The field of action recognition is also vast and it has been the focus of much of the recent research. Since action recognition is not the focus of this thesis, this section does not intend to provide a thorough explanation of the current state of the art. Instead, it will give an overview of some relevant research areas to contextualize our work. A more complete review of action recognition methods can be found in the paper by Aggarwal and Ryoo (2011).

Most of action recognition works focus on action classification, which consists in providing a label to a video or image containing one single action being performed. Examples of works in this field include those by Schüldt et al. (2004), Blank et al. (2005) and Wang and Schmid (2013). The first work extracts spatio-temporal invariant points from the videos. These points correspond to an extension of Harris points (Harris and Stephens (1988)) to the 3D temporal domain. Each point is described by computing spatio-temporal Gaussian derivatives in its neighborhood, which are then clustered using k-means. The clusters can be interpreted 
as a codebook, used in the classical bag-of-words approach (Yang et al. (2007)). Having the codebook, each sequence can be described by a histogram of clusters and then classified using a SVM. The paper by Blank et al. (2005) represents the actions as temporal volumetric shapes. Each action video is represented by a cube containing the volumetric shape described by local and global features, including saliency and orientation. The cubes are then classified by using the Nearest Neighbors procedure. One popular approach that has recently shown promising results is the use of trajectories to classify action sequences. A trajectory is obtained by following a point of interest throughout the video. Wang and Schmid (2013) obtain trajectories from dense optical flow (Farnebäck (2003)). In order to deal with camera motion, a homography is estimated between consecutive frames, and the trajectories that follow the same motion pattern of the camera are removed to avoid clutter. Each trajectory is described by segmenting it into a sequence of spatio-temporal cubes. The area inside each cube is described by histograms of gradients and optical flows. The videos are then classified using the bag of words approach (Yang et al. (2007)) with a linear SVM.

In this work, however, we are interested in dealing with another class of problems, where many actions may be performed simultaneously. Furthermore, the actions performed by each person may also vary over time. This kind of situation is related to the action localization problem. Action localization consists in finding all the instances of a given action in a longer video. Laptev and Pérez (2007) do so by formulating event detection as an object detection problem. The "objects" consist of small sequences with various configurations of spatial positioning and temporal length extracted from the videos, which are described by spatio-temporal histograms of gradients and optical flows. The sequences are then classified using a set of weak classifiers obtained by Adaboost (Viola and Jones (2004)). In the paper by Lu and Little (2006), the authors propose a joint tracking and action recognition framework where both are treated simultaneously. Thus, the action location is obtained by tracking the target from the first frame. Afterwards, the sequence of tracking results is used to classify the action by means of a set of Hidden Markov Models (HMM). The action recognition module implemented in this thesis is inspired by this work and follows a similar approach. 


\section{Chapter 3}

\section{Multi-object tracking based on structural information}

In this chapter the proposed tracking framework is explained. The tracking relies on the following assumptions:

- A representative set of annotated examples is available for training the model;

- The number of tracked objects is fixed and it is specified beforehand;

- The topology of the graph is predetermined by a provided adjacency matrix;

- The initial position of each object is provided in the first frame;

- There exists a structural relation pattern between the objects that can be learned and explored for tracking.

The proposed method presents some interesting advantages. Firstly, it performs online tracking, i.e. it only depends on past information and, therefore, it may be used on real time applications. Secondly, the probabilistic graph model is very flexible and, provided that the training set is representative enough, it is able to cover a wide range of configurations. The graph is also able to encode specific structural relations, e.g. it can enforce that object 1 is always to the left of object 2, simply by not providing any training example where this configuration is not followed. Redetecting a lost target can also be done efficiently by restricting the search space to areas that are according to the learned structure. As shall be presented in the experimental results, this redection step significantly amends the drift problem.

\section{$3.1 \quad$ Framework}

Figure 3.1 shows a flowchart of the process, which is based on particle filters and graphs. 


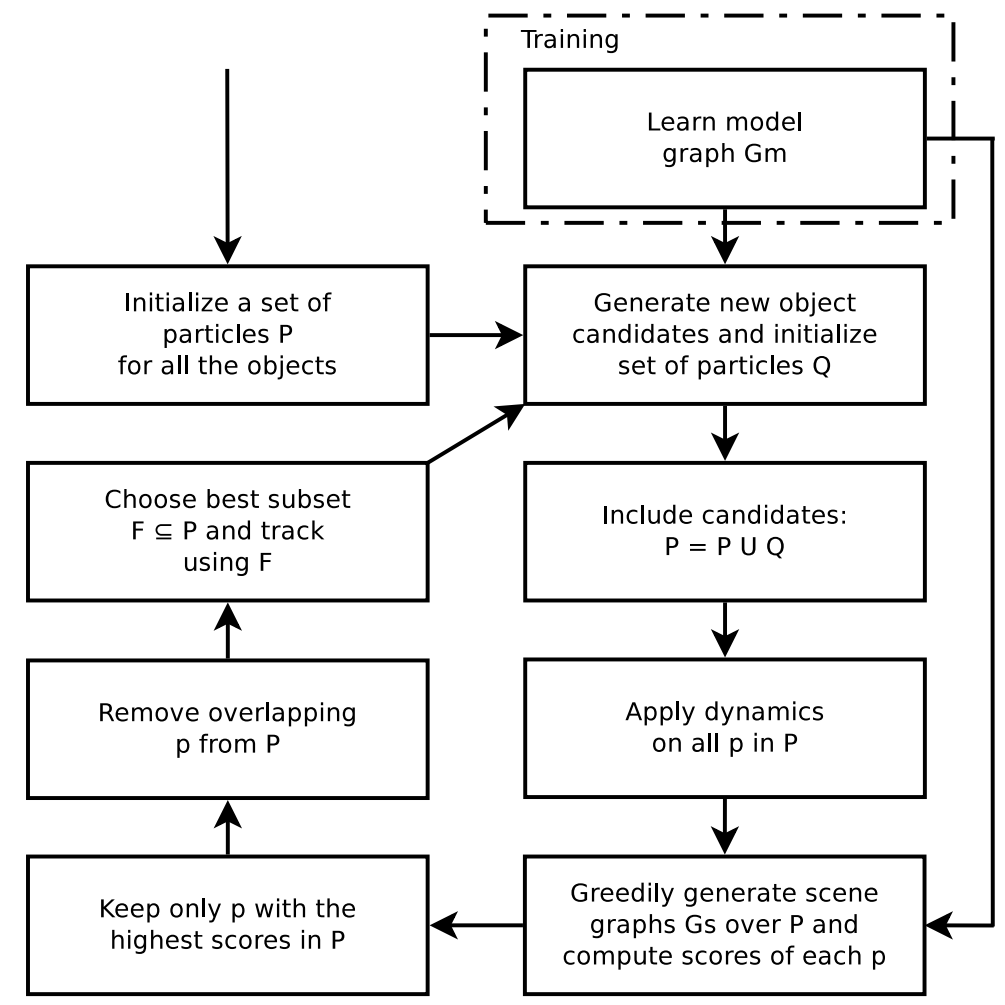

Figure 3.1: Overview of the proposed framework.

First, the model graph $G^{M}$ of the image structure is learned using annotated training images as described in Section 3.3.2. For the tracking step, the position of each of the $N_{O}$ objects can be either manually annotated in the first frame of the video or obtained automatically using detectors. In this work, we adopt the former option. Multiple hypotheses about the state of each object $i$ are kept by using a set of trackers

$$
\mathcal{P}_{i}^{t}=\left\{\left(P_{j}^{i}, w_{j}^{i}\right) \mid j=1, \ldots, n_{i}^{t}\right\}
$$

where $P_{j}^{i}$ is the $j$-th tracker of object $i, w_{j}^{i}$ is a temporal confidence score and $n_{i}^{t}$ represents the number of trackers for object $i$ at instant $t$.

Abrupt motion is usually caused by a sudden change in speed or direction of the target. It can also happen when the object enters an occluded area, such as behind a wall or outside the field of view of the camera. Another reason is either in case the camera is cut off for a moment, or when the same scene is observed from another point of view. These events will be referred to as camera cuts. Abrupt motion is handled by continuously generating new hypotheses about the position of each target. For this, $G^{M}$ is used to generate candidates on the most likely locations (Section 3.4.1). Each candidate yields a new pair $\left(P_{k}^{i}, w_{k}^{i}=0\right)$ which is then added to $\mathcal{P}_{i}^{t}$. All trackers in $\mathcal{P}_{i}^{t}$ are then updated by applying their respective state dynamics.

After including the candidates in the set, a temporal score is computed for every $P_{j}^{i} \in \mathcal{P}_{i}^{t}$ 
(Section 3.4.2). This is done by using a greedy approach to generate the scene graphs $G_{k}^{S}$ and evaluating them using the model graph $G^{M}$ (Section 3.4.3). The scores are then used to remove undesired trackers from the set (Section 3.4.4). The final step consists in actually choosing the best trackers from each set to provide the final multi-object tracking result (Section 3.4.5). The next sections detail each step.

\subsection{Tracking objects with particle filters}

In this section the standard method of tracking with particle filters is presented. Particle filter is an interesting method because it is able to deal with non-Gaussian and non-linear models, as opposed to Kalman filters. For this reason, as evidenced by the works presented in Chapter 2, many recent researches on tracking rely on particle filters.

\subsubsection{Bayes recursive filter}

Particle filter comes from the Bayes recursive filter. When the Bayes filtering function cannot be solved analytically, particles are used to provide a numerical approximation. Therefore, before presenting the particle filter, the Bayes recursive filter will be explained.

\section{Statistics fundamentals}

For completeness, some basic statistical properties that are used for deriving the filtering function will be presented. For more details or proofs, the reader is referred to Bussab and Morettin (2010). The most important definition is the Bayes' theorem. Given random variables $X$ and $Y$, their conditional probability can be computed by

$$
\mathbb{P}(X \mid Y)=\frac{\mathbb{P}(Y \mid X) \mathbb{P}(X)}{\mathbb{P}(Y)}
$$

The left-hand side of this equation is usually called posterior. On the right-hand side, the denominator is referred to as evidence, while the numerator is composed of the likelihood and the prior, respectively.

The joint probability of two variables can be expressed as

$$
\mathbb{P}(X, Y)=\mathbb{P}(X \mid Y) \mathbb{P}(Y)
$$

The same properties are still valid when conditioning the previous variables to another one $Z$ :

$$
\mathbb{P}(X \mid Y, Z)=\frac{\mathbb{P}(Y \mid X, Z) \mathbb{P}(X \mid Z)}{\mathbb{P}(Y \mid Z)} .
$$




$$
\mathbb{P}(X, Y \mid Z)=\mathbb{P}(X \mid Y, Z) \mathbb{P}(Y \mid Z) .
$$

Finally, the marginal distribution of $X$ over $Y$ is given by:

$$
\mathbb{P}(X)=\int_{Y \in \mathcal{Y}} \mathbb{P}(X, Y) d Y
$$

\section{Bayes filter}

A classical filtering problem operates over a Hidden Markov Model (Section 4.3). Let $\mathcal{X}$ and $\mathcal{O}$ be the sets of states and observations, respectively. Let also $\boldsymbol{x}_{t} \in \mathcal{X}$ be a hidden state at instant $t$ and $\boldsymbol{o}_{t} \in \mathcal{O}$ an observation emitted by $\boldsymbol{x}_{t}$. It is assumed that the model is a Markov process of first order (Section 4.3) and it is conditionally independent of the joint of previous states and observations:

$$
\mathbb{P}\left(\boldsymbol{x}_{t} \mid \boldsymbol{x}_{1: t-1}, \boldsymbol{o}_{1: t}\right)=\mathbb{P}\left(\boldsymbol{x}_{t} \mid \boldsymbol{x}_{t-1}\right),
$$

where $\boldsymbol{x}_{1: t-1}$ denotes a sequence of states from instant 1 until $t-1$, and similarly for $\boldsymbol{o}_{1: t}$. In the same sense, the observation is also conditionally independent from the joint of previous states and observations:

$$
\mathbb{P}\left(\boldsymbol{o}_{t} \mid \boldsymbol{x}_{1: t}, \boldsymbol{o}_{1: t-1}\right)=\mathbb{P}\left(\boldsymbol{o}_{t} \mid \boldsymbol{x}_{t}\right)
$$

The filtering problem consists in estimating the posterior distribution $\mathbb{P}\left(\boldsymbol{x}_{t} \mid \boldsymbol{o}_{1: t}\right)$ using the Bayes' theorem:

$$
\mathbb{P}\left(\boldsymbol{x}_{t} \mid \boldsymbol{o}_{1: t}\right)=\frac{\mathbb{P}\left(\boldsymbol{o}_{1: t} \mid \boldsymbol{x}_{t}\right) \mathbb{P}\left(\boldsymbol{x}_{t}\right)}{\mathbb{P}\left(\boldsymbol{o}_{1: t}\right)} .
$$

This can be done by deriving this equation to use the posterior obtained at instant $t-1$ as a prior at instant $t$, as follows:

$$
\begin{aligned}
\mathbb{P}\left(\boldsymbol{x}_{t} \mid \boldsymbol{o}_{1: t}\right) & =\frac{\mathbb{P}\left(\boldsymbol{o}_{1: t} \mid \boldsymbol{x}_{t}\right) \mathbb{P}\left(\boldsymbol{x}_{t}\right)}{\mathbb{P}\left(\boldsymbol{o}_{1: t}\right)} \\
& =\frac{\mathbb{P}\left(\boldsymbol{o}_{t}, \boldsymbol{o}_{1: t-1} \mid \boldsymbol{x}_{t}\right) \mathbb{P}\left(\boldsymbol{x}_{t}\right)}{\mathbb{P}\left(\boldsymbol{o}_{t}, \boldsymbol{o}_{1: t-1}\right)}
\end{aligned}
$$

using the property of Equation 3.5:

$$
=\frac{\mathbb{P}\left(\boldsymbol{o}_{t} \mid \boldsymbol{o}_{1: t-1}, \boldsymbol{x}_{t}\right) \mathbb{P}\left(\boldsymbol{o}_{1: t-1} \mid \boldsymbol{x}_{t}\right) \mathbb{P}\left(\boldsymbol{x}_{t}\right)}{\mathbb{P}\left(\boldsymbol{o}_{t} \mid \boldsymbol{o}_{1: t-1}\right) \mathbb{P}\left(\boldsymbol{o}_{1: t-1}\right)}
$$


now, by applying the Bayes' theorem on the second term of the numerator:

$$
\begin{aligned}
& =\frac{\mathbb{P}\left(\boldsymbol{o}_{t} \mid \boldsymbol{o}_{1: t-1}, \boldsymbol{x}_{t}\right) \mathbb{P}\left(\boldsymbol{x}_{t} \mid \boldsymbol{o}_{1: t-1}\right) \mathbb{P}\left(\boldsymbol{o}_{1: t-1}\right) \mathbb{P}\left(\boldsymbol{x}_{t}\right)}{\mathbb{P}\left(\boldsymbol{o}_{t} \mid \boldsymbol{o}_{1: t-1}\right) \mathbb{P}\left(\boldsymbol{o}_{1: t-1}\right) \mathbb{P}\left(\boldsymbol{x}_{t}\right)} \\
& =\frac{\mathbb{P}\left(\boldsymbol{o}_{t} \mid \boldsymbol{o}_{1: t-1}, \boldsymbol{x}_{t}\right) \mathbb{P}\left(\boldsymbol{x}_{t} \mid \boldsymbol{o}_{1: t-1}\right)}{\mathbb{P}\left(\boldsymbol{o}_{t} \mid \boldsymbol{o}_{1: t-1}\right)}
\end{aligned}
$$

finally, according to Equation 3.8:

$$
=\frac{\mathbb{P}\left(\boldsymbol{o}_{t} \mid \boldsymbol{x}_{t}\right) \mathbb{P}\left(\boldsymbol{x}_{t} \mid \boldsymbol{o}_{1: t-1}\right)}{\mathbb{P}\left(\boldsymbol{o}_{t} \mid \boldsymbol{o}_{1: t-1}\right)} .
$$

The prior can be further derived in terms of a marginal distribution over the previous state:

$$
\mathbb{P}\left(\boldsymbol{x}_{t} \mid \boldsymbol{o}_{1: t-1}\right)=\int \mathbb{P}\left(\boldsymbol{x}_{t}, \boldsymbol{x}_{t-1} \mid \boldsymbol{o}_{1: t-1}\right) d \boldsymbol{x}_{t-1}
$$

using the property of Equation 3.5:

$$
=\int \mathbb{P}\left(\boldsymbol{x}_{t} \mid \boldsymbol{x}_{t-1}, \boldsymbol{o}_{1: t-1}\right) \mathbb{P}\left(\boldsymbol{x}_{t-1} \mid \boldsymbol{o}_{1: t-1}\right) d \boldsymbol{x}_{t-1}
$$

by applying the conditional independence of Equation 3.7:

$$
=\int \mathbb{P}\left(\boldsymbol{x}_{t} \mid \boldsymbol{x}_{t-1}\right) \mathbb{P}\left(\boldsymbol{x}_{t-1} \mid \boldsymbol{o}_{1: t-1}\right) d \boldsymbol{x}_{t-1}
$$

By doing so the previous posterior can be directly applied onto the equation to estimate the new value. The evidence of Equation 3.10 can also be expressed in terms of a marginal distribution:

$$
\begin{aligned}
\mathbb{P}\left(\boldsymbol{o}_{t} \mid \boldsymbol{o}_{1: t-1}\right) & =\int \mathbb{P}\left(\boldsymbol{o}_{t}, \boldsymbol{x}_{t} \mid \boldsymbol{o}_{1: t-1}\right) d \boldsymbol{x}_{t} \\
& =\int \mathbb{P}\left(\boldsymbol{o}_{t} \mid \boldsymbol{x}_{t}, \boldsymbol{o}_{1: t-1}\right) \mathbb{P}\left(\boldsymbol{x}_{t} \mid \boldsymbol{o}_{1: t-1}\right) d \boldsymbol{x}_{t} \\
& =\int \mathbb{P}\left(\boldsymbol{o}_{t} \mid \boldsymbol{x}_{t}\right) \mathbb{P}\left(\boldsymbol{x}_{t} \mid \boldsymbol{o}_{1: t-1}\right) d \boldsymbol{x}_{t} .
\end{aligned}
$$

Replacing Equations 3.11 and 3.12 into Equation 3.10, we obtain the final form of the filter:

$$
\mathbb{P}\left(\boldsymbol{x}_{t} \mid \boldsymbol{o}_{1: t}\right)=\frac{\mathbb{P}\left(\boldsymbol{o}_{t} \mid \boldsymbol{x}_{t}\right) \int \mathbb{P}\left(\boldsymbol{x}_{t} \mid \boldsymbol{x}_{t-1}\right) \mathbb{P}\left(\boldsymbol{x}_{t-1} \mid \boldsymbol{o}_{1: t-1}\right) d \boldsymbol{x}_{t-1}}{\int \mathbb{P}\left(\boldsymbol{o}_{t} \mid \boldsymbol{x}_{t}\right) \mathbb{P}\left(\boldsymbol{x}_{t} \mid \boldsymbol{o}_{1: t-1}\right) d \boldsymbol{x}_{t}}
$$

Except if the system presents some properties such as Gaussian distributions and linear models, Equation 3.10 cannot be solved analytically. When the system is more complex, then the result can only be approximated using, for example, a particle filter. 


\subsubsection{Particle filter}

Let $\boldsymbol{x}_{t}^{i}$ be a hypothetical realization of the state $\boldsymbol{x}_{t}$ and $\delta\left(\boldsymbol{x}_{t}-\boldsymbol{x}_{t}^{i}\right)$ be the Dirac delta function centered on $\boldsymbol{x}_{t}^{i}$. A particle filter solves the filtering problem by approximating the posterior $\mathbb{P}\left(\boldsymbol{x}_{t} \mid \boldsymbol{o}_{1: t}\right)$ by a weighted sum of $N_{P}$ Dirac masses:

$$
\mathbb{P}\left(\boldsymbol{x}_{t} \mid \boldsymbol{o}_{1: t}\right)=\sum_{i=1}^{N_{P}} \eta_{t}^{i} \delta\left(\boldsymbol{x}_{t}-\boldsymbol{x}_{t}^{i}\right),
$$

where each $\boldsymbol{x}_{t}^{i}$ is called a particle with associated weight $\eta_{t}^{i}$.

This work employs particle filter using the ConDensation algorithm, which uses factored sampling (Isard and Blake (1998)) to update the particles. The cloud of particles $P_{t}=$ $\left\{\left(\boldsymbol{x}_{t}^{i}, \eta_{t}^{i}\right) \mid i=1, \ldots, N_{P}\right\}$ is obtained by resampling them, with repositions, from the past one $P_{t-1}=\left\{\left(\boldsymbol{x}_{t-1}^{i}, \eta_{t-1}^{i}\right) \mid i=1, \ldots, N_{P}\right\}$. By assuming $\sum_{j=1}^{N_{P}} \eta_{t-1}^{j}=1$, the probability of each particle $i$ being chosen in this step is $\eta_{t-1}^{i}$. Hence, more likely particles can be sampled several times, while others may not be chosen at all.

The particles are then propagated according to a proposal function $\boldsymbol{x}_{t}^{i} \sim q\left(\boldsymbol{x}_{t} \mid \boldsymbol{x}_{0: t-1}^{i}, \boldsymbol{o}_{1: t}\right)$, which is usually assumed to be the dynamics model $\mathbb{P}\left(\boldsymbol{x}_{t} \mid \boldsymbol{x}_{t-1}\right)$, yielding $\boldsymbol{x}_{t}^{i} \sim \mathbb{P}\left(\boldsymbol{x}_{t} \mid \boldsymbol{x}_{t-1}\right)$. The propagation phase involves two steps: drift and diffusion. Drift is a deterministic step, which consists in applying the motion dynamics for each particle. Diffusion, on the other hand, is random and it is used to include noise in the model. The new state of a particle $i$ can be expressed as:

$$
\boldsymbol{x}_{t}^{i}=\boldsymbol{D} \boldsymbol{x}_{t-1}^{i}+\boldsymbol{U}
$$

where $\boldsymbol{D}$ is the motion dynamics and $\boldsymbol{U}$ is the noise matrix.

Finally, the weights of the particles are updated according to the new observations $\boldsymbol{o}_{t}$ as:

$$
\eta_{t}^{i}=\frac{\eta_{t-1}^{i}}{\sum_{j=1}^{N_{P}} \eta_{t}^{j}} \frac{\mathbb{P}\left(\boldsymbol{o}_{t} \mid \boldsymbol{x}_{t}^{i}\right) \mathbb{P}\left(\boldsymbol{x}_{t} \mid \boldsymbol{x}_{t-1}\right)}{q\left(\boldsymbol{x}_{t} \mid \boldsymbol{x}_{0: t-1}^{i}, \boldsymbol{o}_{1: t}\right)} .
$$

If the proposal function is the dynamics model, the weight update is simplified to:

$$
\eta_{t}^{i}=\frac{\eta_{t-1}^{i}}{\sum_{j=1}^{N_{P}} \eta_{t}^{j}} \mathrm{P}\left(\boldsymbol{o}_{t} \mid \boldsymbol{x}_{t}^{i}\right)
$$

The final estimated state of a cloud of particle $P$ may be computed using several heuristics, but the most widely used is by computing the weighted average:

$$
r(P)=\boldsymbol{x}_{t}=\sum_{i=1}^{N_{P}} \eta_{t}^{i} \boldsymbol{x}_{t}^{i}
$$

In this work, we are also interested in evaluating the overall quality of a cloud $P$. We 
propose to do this by computing a confidence score based on the non-normalized weights of the particles:

$$
\zeta(P)=1-\exp \left(-\sum_{i=1}^{N_{P}} \eta_{t-1}^{i} \mathbb{P}\left(\boldsymbol{o}_{t} \mid \boldsymbol{x}_{t}^{i}\right)\right)
$$

\subsubsection{State and dynamics models}

In this work, the objects to be tracked are represented by rectangular bounding boxes. Each box is parameterized by its centroid and two measures: height and width. It is assumed that the variation in scale is not significant. Therefore, the state of each particle is given by a column vector $\boldsymbol{x}_{t}^{i}=\left(x_{t}^{i}, y_{t}^{i}\right)^{T}$, which represents one candidate centroid. The motion model is a random walk, i.e. $\boldsymbol{D}=\boldsymbol{I}$ and $\boldsymbol{U}=\boldsymbol{I} \boldsymbol{u}$, yielding:

$$
\boldsymbol{x}_{t}^{i}=\boldsymbol{x}_{t-1}^{i}+\boldsymbol{u}
$$

where $\boldsymbol{I}$ is the identity matrix and $\boldsymbol{u}=\left(u_{x}, u_{y}\right)^{T}$ such that $u_{x}$ and $u_{y}$ are noise terms that follow a Gaussian distribution with zero mean.

More complex states and motion models could be used. For example, the state could also include additional information such as the velocity, acceleration, orientation, scale and so on. The greatest problem when considering more information is that each additional parameter increases the search space in one dimension. Therefore, the number of particles necessary to cover a significant part of this larger space increases exponentially. As when tracking multiple objects the amount of particles necessary to track all of them may increase fast, it was chosen to use the least amount of particles that produced good tracking results.

\subsubsection{Color histogram-based tracking}

The objects are tracked using color histograms as proposed by Pérez et al. (2002). The method works by using color information obtained from the HSV color space. This color model is interesting because it separates the chromatic information (Hue and Saturation) from the shading (Value). However, the authors point out that the chromatic information is only reliable when both the saturation and the value are not too low. Therefore, first an HS histogram with $N_{H} N_{S}$ bins is populated using only information obtained from pixels whose Saturation and Value are above a given threshold of 0.1 and 0.2 , respectively. The remaining pixels are not discarded, because their information can be useful when dealing with images which are mostly black and white. Those pixels are used to populate a $\mathrm{V}$ histogram that is concatenated to the HS one built before. The resulting histogram is composed of $N_{H} N_{S}+N_{V}$ bins. Following the original paper, the variables are set as $N_{H}=N_{S}=N_{V}=10$.

Each histogram corresponds to one observation $\boldsymbol{o}_{t}^{j}$ for object $j$ at instant $t$ for the particle filter. Section 3.4.2 presents more details about how the histograms are compared in order 
to track each object.

\subsection{Building the graphs}

\subsubsection{Attributed Relational Graph (ARG)}

An ARG is a tuple

$$
G=\left(\mathcal{V}, \mathcal{E}, \mathcal{A}_{\mathcal{V}}, \mathcal{A}_{\mathcal{E}}\right)
$$

where $\mathcal{V}=\left\{v_{i} \mid i=1, \ldots, N_{O}\right\}$ represents a set of vertices (or nodes), $\mathcal{E}=\left\{e_{i j}=\left(v_{i}, v_{j}\right) \mid i, j=\right.$ $\left.1, \ldots, N_{O}\right\}$ is a set of directed edges (or arcs), i.e. $e_{i j} \neq e_{j i}$ and $\mathcal{A}_{\mathcal{V}}$ and $\mathcal{A}_{\mathcal{E}}$ are sets of attributes of the vertices and the arcs, respectively.

Each frame of the video (also referred to as scene) is represented by one or more ARGs. The vertices of $G$ are the tracked objects, while the edges connect objects whose relations will be analyzed. The desired relations are expressed using a binary adjacency matrix $M_{A}=\left(m_{i j}\right)$ where $m_{i j}=1$ if there is an edge from $v_{i}$ to $v_{j}$. Figure 3.2 shows one possible scene graph generated from the adjacency matrix:

$$
M_{A}=\left(\begin{array}{lllll}
0 & 1 & 0 & 0 & 1 \\
1 & 0 & 0 & 0 & 1 \\
0 & 0 & 0 & 1 & 1 \\
0 & 0 & 1 & 0 & 1 \\
1 & 1 & 1 & 1 & 0
\end{array}\right)
$$

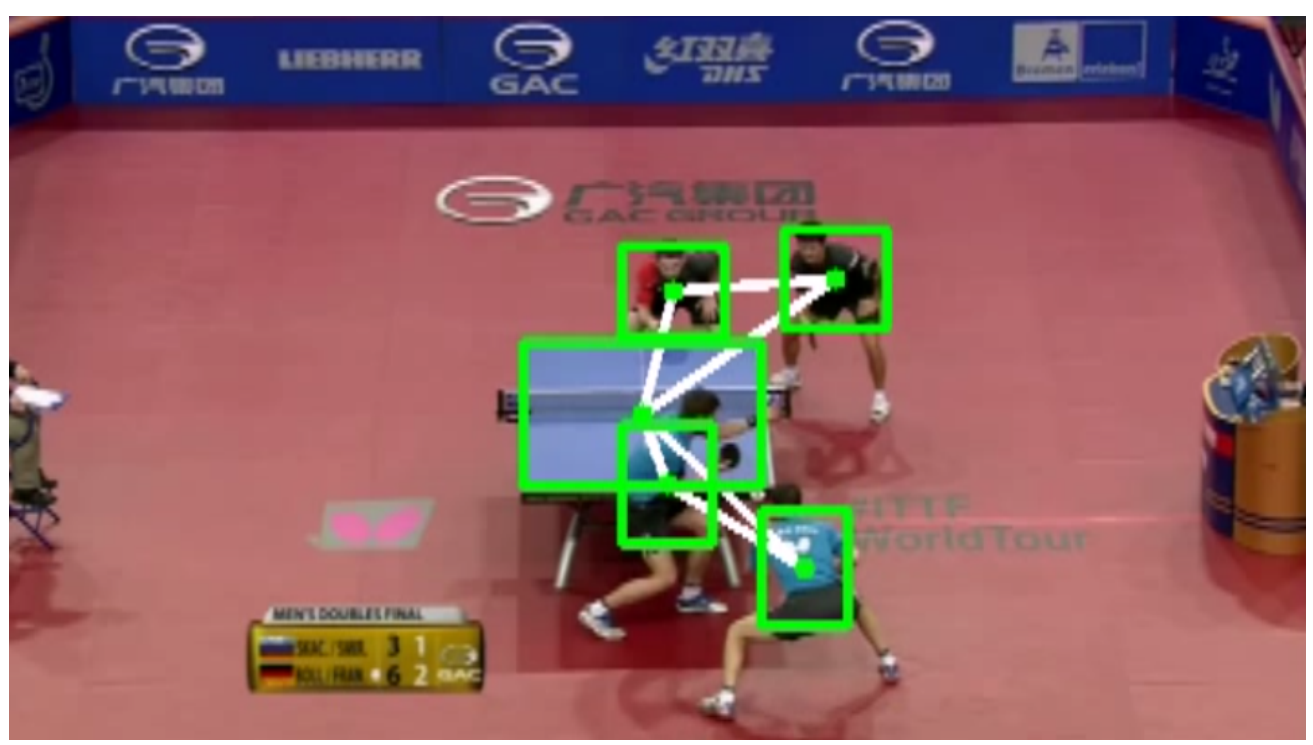

Figure 3.2: An example of a scene graph.

Two different kinds of attributes are used: the appearance and the structural attributes. 
Appearance attributes are related to each object and they are stored in $\mathcal{A}_{\mathcal{V}}$. On the other hand, structural attributes represent relations among objects, and thus constitute edge attributes in $\mathcal{A}_{\mathcal{E}}$.

\subsubsection{Model graph}

The topology of $G^{M}$ is obtained by means of an adjacency matrix $M_{A}$, which must be provided by the user. The set of attributes $\mathcal{A}_{\mathcal{E}}^{M}$ of $G^{M}$ is computed from a database of annotated images. Each image is labeled with the state of each relevant object (typically a surrounding bounding box and an object label). Let $\delta_{k} \in \Delta(i, j)$ be one of the structural attributes to be measured (e.g. the distance between two objects). The annotations are used to estimate the probability density function (PDF) of $\delta_{k}$. Inspired by Cho et al. (2013), the set of chosen attributes is

$$
\Delta(i, j)=\left\{\left(\theta\left(e_{i j}\right), d\left(v_{i}, v_{j}\right)\right)\right\}
$$

and the PDF is estimated by means of histograms $H_{\delta_{k}}$. The function $\theta\left(e_{i j}\right)$ represents the clockwise angle between the horizontal axis and the vector $\overrightarrow{v_{i} v_{j}}$ and $d\left(v_{i}, v_{j}\right)$ is the distance between the two vertices (Fig. 3.3).

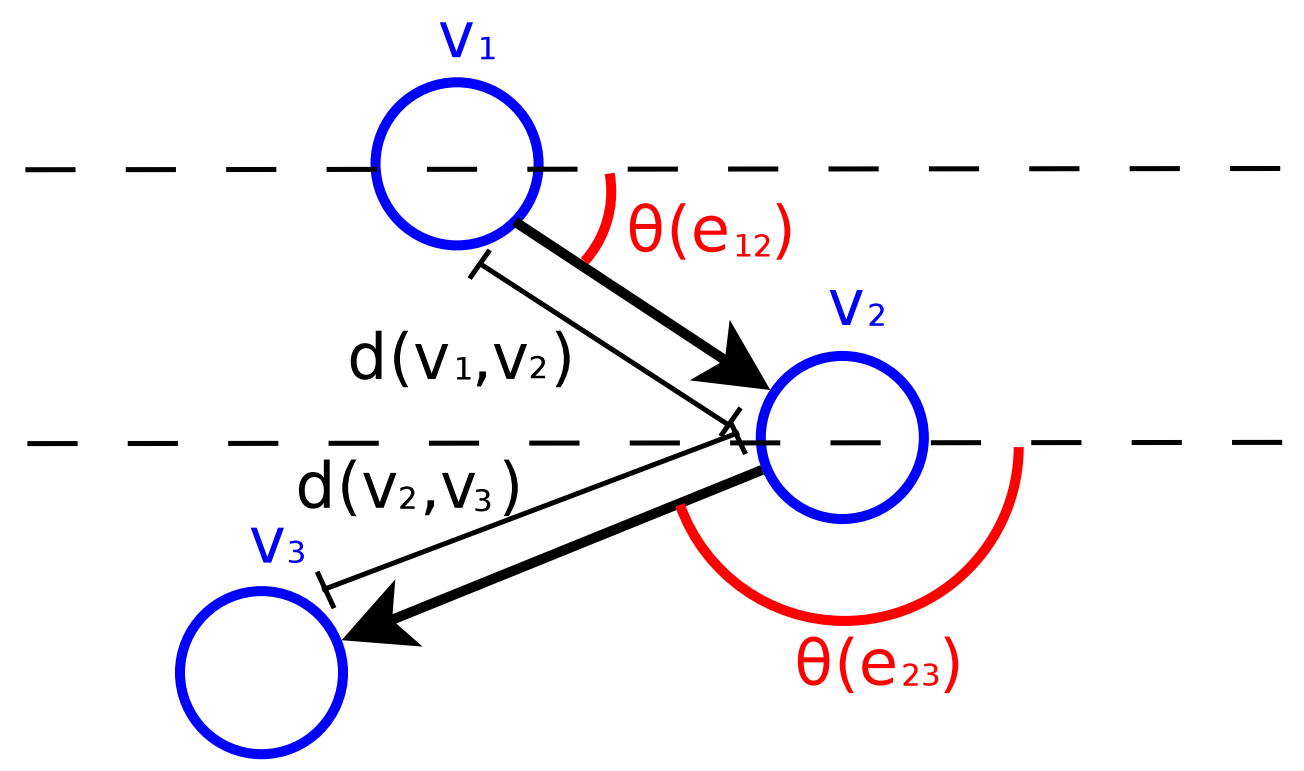

Figure 3.3: The structural attributes of the edges.

The histograms are built by iterating over all images and collecting the respective observations $o$, which cast a vote for the histogram bin $H_{\delta_{k}}(o)$. The PDF is then estimated by normalizing $H_{\delta_{k}}$ to have a sum equal to one. Finally, the normalized histograms are then used as the arc attributes $\mathcal{A}_{\mathcal{E}}^{M}=\left\{H_{\delta_{k}} \mid \delta_{k} \in \Delta(i, j)\right\}$. Since histograms suffer from the discretization problem, other approaches such as Kernel Density Estimation (Elgammal et al. (2000)) or Gaussian Mixture Model (Karavasilis et al. (2015)) could be used instead. How- 
ever, the former may require a large amount of memory in case the training set is big, while the latter either relies on setting a number of mixtures beforehand or requires a more complex training (Blei et al. (2006)). Therefore, given the simplicity of histograms and the good results observed in the experiments, we chose to use them to represent the distributions.

The appearance attributes in $\mathcal{A}_{\mathcal{V}}^{M}$ are not learned from the database. Instead, they are computed from annotations provided in the first frame of the tracking video. In our experiments, the appearance was described by using color histograms. However, any other appearance descriptor could also be considered, like HOG (Dalal and Triggs (2005)) or SIFT (Lowe (2004)). Figure 3.4 shows one graph and the learned histograms for each attribute.

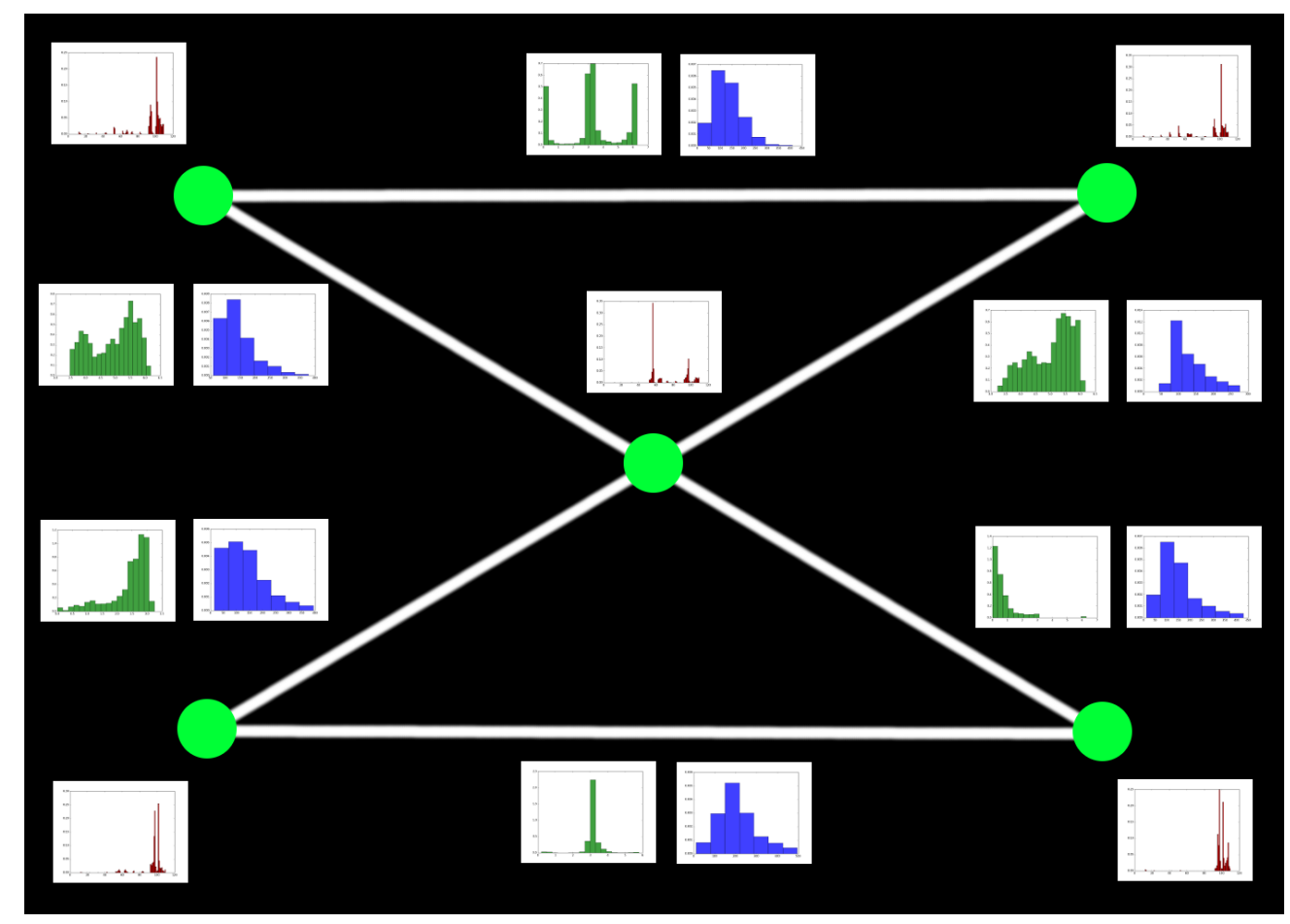

Figure 3.4: An example of a model graph with the learned attributes. The red histograms represent the vertices attributes (color model), while the green ones represent the angles and the blue ones represent the distances.

\subsubsection{Scene graph}

Each graph $G_{k}^{S}$ of index $k$ represents a different scene configuration. A vertex $v_{i} \in \mathcal{V}^{S}$ of the scene graph $G_{k}^{S}$ is associated with one cloud of particles $P_{j}^{i}$ for object $i$. Let $r\left(P_{j}^{i}\right)=$ $\left(x_{1}^{i, j}, x_{2}^{i, j}, \ldots, x_{|\mathcal{X}|}^{i, j}\right)$ represent the final vector state obtained from Equation 3.18 for the particle cloud $P_{j}^{i}$. Assuming that $x_{1}^{i, j}$ and $x_{2}^{i, j}$ represent the $2 \mathrm{D}$ coordinates of the object in the image space, the position of $v_{i}$ is obtained from

$$
r_{P}\left(P_{j}^{i}\right)=\left(x_{1}^{i, j}, x_{2}^{i, j}\right)
$$


i.e. $r_{P}\left(P_{j}^{i}\right)$ is a truncated version of $r\left(P_{j}^{i}\right)$ that only includes the spatial coordinates.

The edges are then produced using the same matrix $M_{A}$ as in the training. However, recall that each object is tracked by a set of different trackers. Therefore, each scene may be described by multiple graphs with the same topology, which represent all the possible combinations of different trackers for each object. If all the possible graphs are generated, then each scene will be represented by $\prod_{i=1}^{N_{O}}\left|\mathcal{P}_{i}^{t}\right|$ graphs. All these graphs could be evaluated and the best one chosen according to the model to obtain the final tracking result, but this could be computationally expensive due to the large number of combinations. Therefore, in this work a different approach is used, which is explained in Section 3.4.3.

The set of structural attributes $\mathcal{A}^{s}$ of $G^{S}$ is not composed of PDFs as in $G^{M}$, but of single values for each measurement $\delta_{l}$ extracted from the current frame (i.e. the observations of $\left.\delta_{k}\right)$. The attributes of the vertices are the associated pairs $\left(P_{j}^{i}, w_{j}^{i}\right)$.

\subsection{Tracking using graphs}

\subsubsection{Generating new candidates}

Besides for tracking evaluation, the structural information of $G^{M}$ is also used to generate new candidate positions for each tracked object. This step is important to deal with abrupt motion. Since the attributes $\mathcal{A}_{\mathcal{E}}^{M}$ are all relative to the origin of each arc $e_{i j}$, the position of $v_{i}$ must be known. Therefore, it is assumed that the trackers for every object will not all fail at the same time. Good candidates can be generated by selecting the positions given by the best trackers as origins. Candidate generation is controlled by using a matrix $M_{C}=\left(m_{i j}\right)$, where $m_{i j}$ indicates that, if object $i$ is used as reference, then it generates $m_{i j}$ candidates for object $j$.

Let $a_{e_{i j}}^{M}=\left\{H\left(\theta\left(e_{i j}\right)\right), H\left(d\left(v_{i}, v_{j}\right)\right)\right\}$ be the attribute of an edge $e_{i j}$ from $G^{M}$. A candidate $k$ is generated for object $j$ as

$$
\left(\hat{\theta}_{k}=\theta_{k}+u_{\theta}, \hat{d}_{k}=d_{k}+u_{d}\right)
$$

by simulating according to the distributions given by the histograms $\theta_{k} \sim H\left(\theta\left(e_{i j}\right)\right)$ and $d_{k} \sim H\left(d\left(v_{i}, v_{j}\right)\right)$, where $u_{\theta} \sim \mathcal{N}\left(0, \sigma_{\theta}\right)$ and $u_{d} \sim \mathcal{N}\left(0, \sigma_{d}\right)$ are Gaussian noises. Each candidate position is then obtained by

$$
\left(v_{i}(x)+\hat{d}_{k} \cos \left(\hat{\theta}_{k}\right), v_{i}(y)+\hat{d}_{k} \sin \left(\hat{\theta}_{k}\right)\right)
$$

Figure 3.5 shows the candidates generated for each object. The candidates are then used to generate new particle clouds $P_{k}^{j}$ which are inserted in the set $\mathcal{P}_{j}^{t}$. The clouds are initialized by spreading the particles according to a Gaussian distribution centered on the candidate 


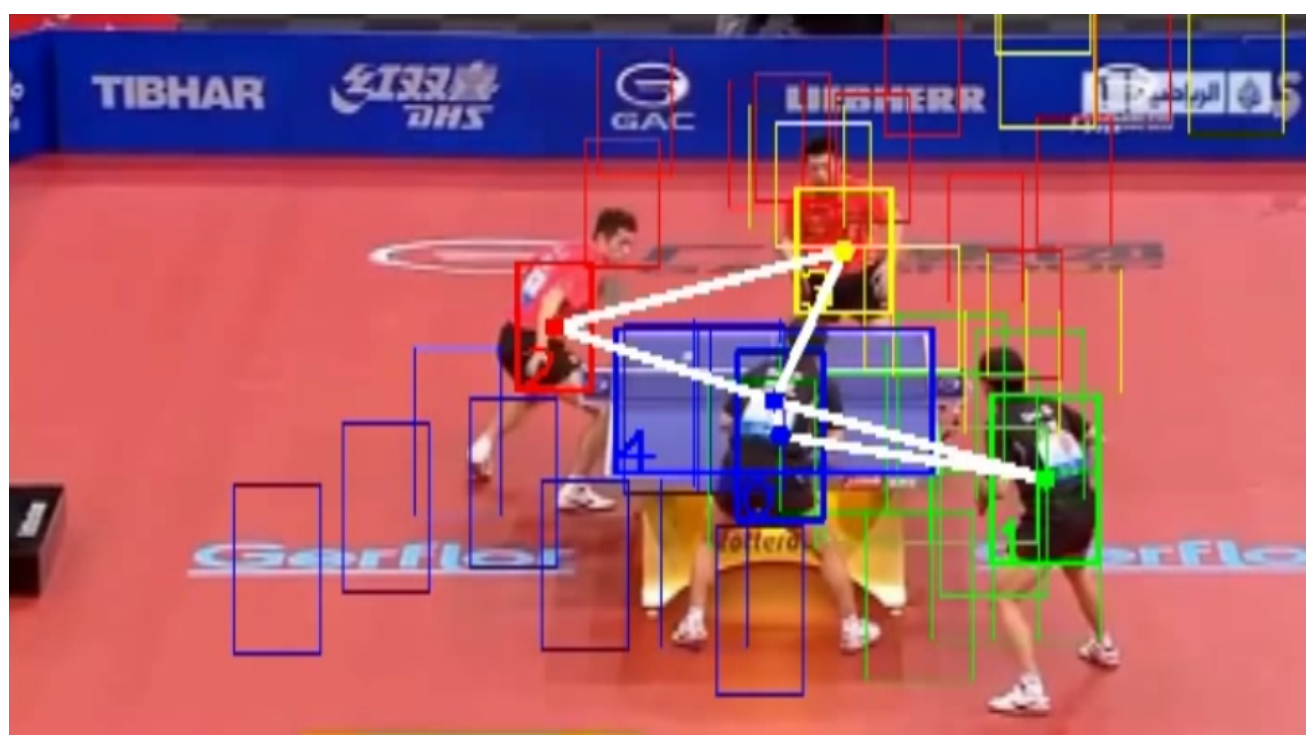

Figure 3.5: Example of candidates generated for one scene. Rectangles of the same color indicate that they belong to the same object.

position.

\subsubsection{Computing temporal scores of trackers}

The temporal score $w^{i}$ measures the reliability of the associated tracker over time. This is done by computing a weighted accumulation of instantaneous scores:

$$
\left(w^{i}\right)^{t}=\rho_{T}\left(w^{i}\right)^{t-1}+f\left(i, G^{S}, G^{M}\right),
$$

where $\rho_{T}$ is a given constant and $f\left(i, G^{S}, G^{M}\right)$ is the instantaneous score function for the vertex $v_{i}^{S}$, which is associated with $\left(P^{i},\left(w^{i}\right)^{t}\right)$. By doing so, trackers which consistently perform well during longer periods of time have higher scores than those that are only eventually good (usually incorrect trackers).

The instantaneous score is divided into two parts:

$$
f\left(i, G^{S}, G^{M}\right)=\rho_{F} \phi\left(i, G^{S}\right)+\left(1-\rho_{F}\right) \psi\left(i, G^{S}, G^{M}\right)
$$

where $\rho_{F}$ is a given weighting factor and $\phi\left(i, G^{S}\right)$ and $\psi\left(i, G^{S}, G^{M}\right)$ are the appearance and structural score functions of $v_{i}$, respectively. 


\section{Appearance score}

The appearance score is actually the confidence of the particle cloud $P_{i}$ associated with vertex $v_{i}$ as in Equation 3.19. Hence, it is set as

$$
\phi\left(i, G^{S}\right)=\zeta\left(P_{i}\right)
$$

The confidence score depends on the weights of the particles, which are based on the likelihood $\mathbb{P}\left(\boldsymbol{o}_{t} \mid \boldsymbol{x}_{t}^{i}\right)$. The distribution is computed in the same way as in the work by Erdem et al. (2012), using the Bhattacharyya distance $d_{B}$ :

$$
\mathbb{P}\left(\boldsymbol{o}_{t} \mid \boldsymbol{x}_{t}^{i}\right)=\exp \left(-\frac{d_{B}\left(H^{M}, H^{S}\right)^{2}}{2 \sigma^{2}}\right),
$$

where $H^{M}$ and $H^{S}$ are histograms of the model and the scene, respectively and

$$
d_{B}\left(H^{M}, H^{S}\right)=\sqrt{1-\sum_{j} \sqrt{H^{M}(j) H^{S}(j)}},
$$

where $H(j)$ is the $j$-th bin of histogram $H$.

\section{Structural score}

Let $\boldsymbol{m}_{\boldsymbol{i}}$ be a vector representing the line $i$ from the adjacency matrix $M_{A}$ (i.e. corresponding to object $i)$. Let also $\boldsymbol{\theta}_{\boldsymbol{i}}^{\boldsymbol{S}}=\left(H_{\theta}^{M}\left(\theta^{S}\left(e_{i j}\right)\right)\right)_{j=1}^{N_{O}}$ and $\boldsymbol{d}_{\boldsymbol{i}}^{\boldsymbol{S}}=\left(H_{d}^{M}\left(d^{S}\left(v_{i}, v_{j}\right)\right)\right)_{j=1}^{N_{O}}$ be the vectors of the values obtained from the bins of the angle and distance model histograms, respectively, i.e. the likelihoods of each structure measurement. The structure score is computed using the dot product:

$$
\psi\left(i, G^{S}, G^{M}\right)=\frac{1}{2\left\|\boldsymbol{m}_{\boldsymbol{i}}\right\|_{1}} \boldsymbol{m}_{\boldsymbol{i}} \cdot \boldsymbol{\theta}_{\boldsymbol{i}}^{\boldsymbol{S}}+\boldsymbol{m}_{\boldsymbol{i}} \cdot \boldsymbol{d}_{\boldsymbol{i}}^{S}
$$

where $\left\|\boldsymbol{m}_{\boldsymbol{i}}\right\|_{1}$ is the $L_{1}$ norm of $\boldsymbol{m}_{\boldsymbol{i}}$. In other words, this score corresponds to the average of the attributes of the edges originating from $v_{i}$.

\subsubsection{Generating scene graphs for evaluation}

The best trackers are selected by building the scene graphs $G_{k}^{S}$ and computing the scores explained before. Figure 3.6 shows some possible graphs that can be generated from some given candidates. Therefore, one option would be to build all possible graphs and to find the one which maximizes the overall score for every tracker. However, this approach was not chosen for two reasons: (1) the number of graph combinations is usually very large and unfeasible to be processed in real time (Figure 3.7), and (2), although the videos are 
assumed to be structured, it does not necessarily mean that the model graph expresses the best configuration for every scene. Instead, it was chosen a greedy approach that fixes the vertices for all objects except one and optimizes the score for one object at a time.
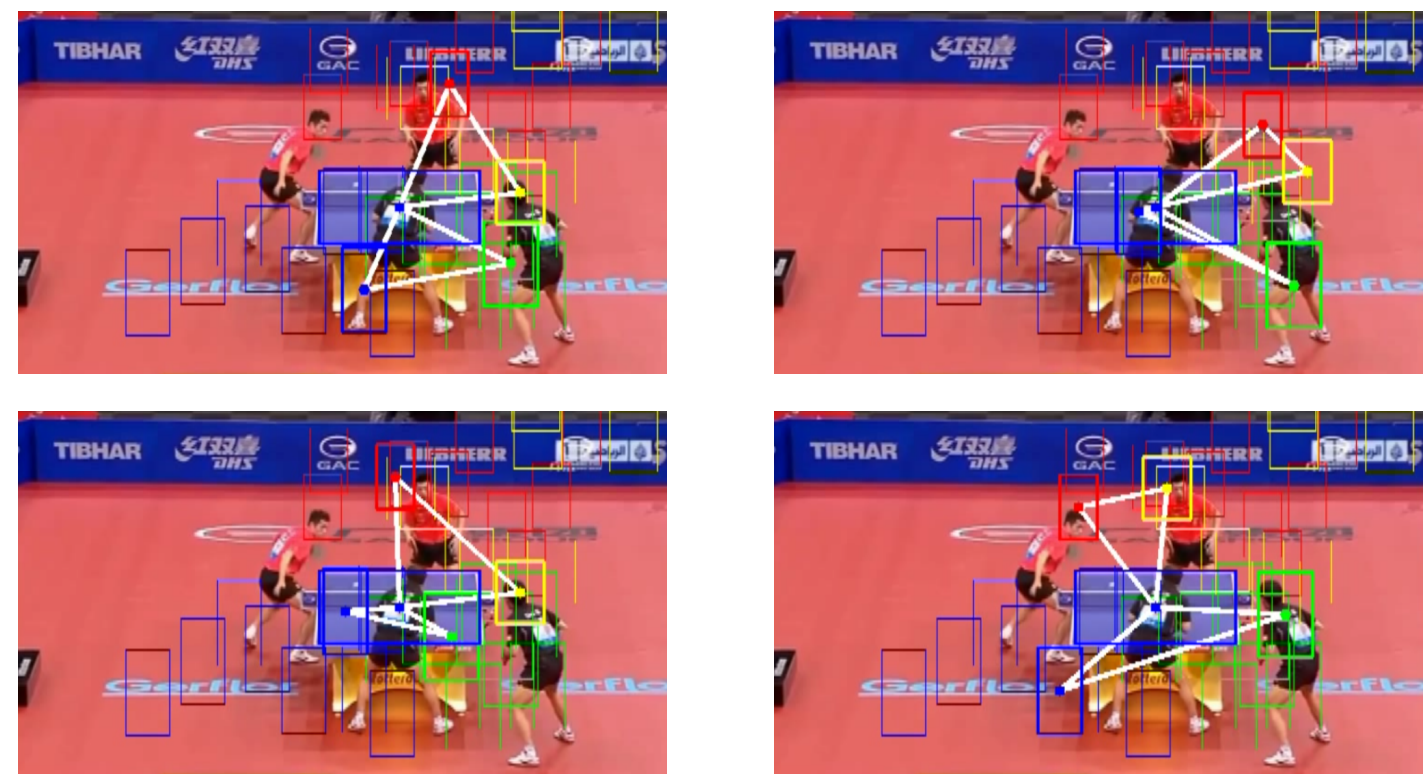

Figure 3.6: Examples of candidate graphs to be analyzed.

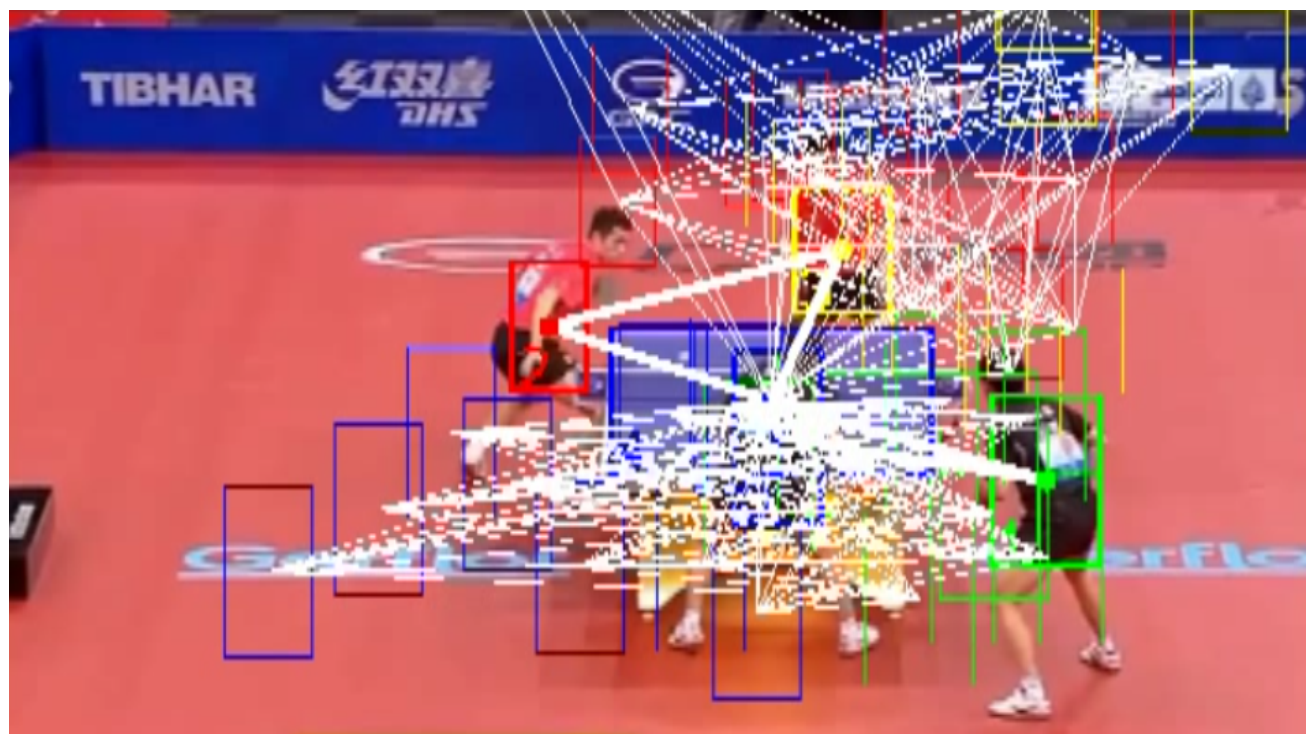

Figure 3.7: Representation of all possible graphs that can be generated from the candidates.

When using a greedy approach, the order in which the objects are processed is important. Let $\left\{\left(P_{*}^{i}, w_{*}^{i}\right) \mid i=1, \ldots, N_{O}\right\}$ be the set of the best trackers of each object, i.e.

$$
\left(P_{*}^{i}, w_{*}^{i}\right)^{t}=\underset{\left(P_{j}^{i}, w_{j}^{i}\right) \in \mathcal{P}_{i}^{t}}{\arg \max } w_{j}^{i}
$$


A sequence is created by sorting $w_{*}^{i}$ in ascending order and processing the objects $i$ one by one according to this sequence. The rationale is that, since all the other vertices will be fixed, it is better to let the worst tracker vary first in order to have good references for the resulting graph. Let $P_{l}^{c}$ be the tracker that is currently being evaluated. This yields a graph $G_{k}^{S}$ whose set of vertices is

$$
\mathcal{V}_{k}^{S}=\left\{v\left(P_{*}^{b}\right) \mid b=1, \ldots, N_{O}\right\} \cup\left\{v\left(P_{l}^{c}\right)\right\} \backslash\left\{v\left(P_{*}^{c}\right)\right\}
$$

where $v(P)$ represents the vertex associated with $P$. This graph is then used to compute the score $w_{l}^{c}$ with $1 \leq c \leq N_{O}$ and $1 \leq l \leq\left|\mathcal{P}_{c}^{t}\right|$.

\subsubsection{Removing undesired trackers}

After computing the score for each tracker, those that are considered as non-significant are removed. This is done by considering two criteria. The first one is thresholding, i.e. removing as many trackers as possible whose scores are too low. More formally, let $\mathcal{Q}_{i}^{(1)}=$ $\left\{\left(P_{j}^{i}, w_{j}^{i}\right)\left|w_{j}^{i}<\tau_{S}, j=1, \ldots,\right| \mathcal{P}_{i}^{t} \mid\right\}$, where $\tau_{S}$ is a given score threshold. The thresholded set is obtained by:

$$
\mathcal{Q}_{i}^{t(2)}= \begin{cases}\left\{\left(P_{*}^{i}, w_{*}^{i}\right)\right\}, & \text { if }\left|\mathcal{P}_{i}^{t}\right|=\left|\mathcal{Q}_{i}^{t(1)}\right| \\ \mathcal{P}_{i}^{t} \backslash \mathcal{Q}_{i}^{t(1)}, & \text { otherwise }\end{cases}
$$

The second criterion relies on the fact that one or more trackers will be representing very similar positions (overlapping) for the same object. In such a case, it is not necessary to keep all of them, because they increase the processing burden and do not introduce much information. It is defined that two trackers $P_{k}^{i}$ and $P_{l}^{i}$ are overlapping when $d\left(r_{P}\left(P_{k}^{i}\right), r_{P}\left(P_{l}^{i}\right)\right)<\tau_{d_{S}}$, where $\tau_{d_{S}}$ is a given overlapping distance threshold for the same object. The distance function may vary depending on the application, but in this thesis we employ the Euclidean distance between vectors. Let $\boldsymbol{q}^{i}=\left(\left(P_{j}^{i}, w_{j}^{i}\right) \mid\left(P_{j}^{i}, w_{j}^{i}\right) \in \mathcal{Q}_{i}^{t(2)}\right)$ be a sequencing of $\mathcal{Q}_{i}^{t(2)}$ sorted in decreasing order of weight. Overlapping trackers are removed by using a greedy approach where the pairs $\left(P_{a}^{i}, w_{a}^{i}\right) \in \boldsymbol{q}^{i}$ are iteratively taken one by one following the ordering and inserted into a new set $\mathcal{Q}_{i}^{t^{(3)}}$ whenever they do not overlap with any existing tracker, i.e. :

$$
\left(\mathcal{Q}_{i}^{t(3)}\right)^{n}= \begin{cases}\left(\mathcal{Q}_{i}^{t(3)}\right)^{n-1} \cup\left\{\left(P_{a}^{i}, w_{a}^{i}\right)\right\}, & \text { if } \forall\left(P_{b}^{i}, w_{b}^{i}\right) \in\left(\mathcal{Q}_{i}^{t(3)}\right)^{n-1} \\ \left(\mathcal{Q}_{i}^{t(3)}\right)^{n-1}, & d\left(r_{P}\left(P_{a}^{i}\right), r_{P}\left(P_{b}^{i}\right)\right) \geq \tau_{d_{S}}\end{cases}
$$

where the exponent $n$ indicates the $n$-th iteration. At the end of this stage, the final set of trackers $\mathcal{P}_{i}^{t+1}=\left(\mathcal{Q}_{i}^{t(3)}\right)^{\mid \mathcal{R}_{i}^{t \mid}}$ is obtained. 


\subsubsection{Choosing the final trackers}

Considering the temporal consistency of videos, it is interesting to try to avoid changing trackers at each frame. However, in order to recover tracking after abrupt motion or appearance ambiguity, it is also necessary to be able to detect when the tracker should be changed. This is done by considering the temporal score of each tracker. For that, let $\left\{\left(\tilde{P}^{i}, \tilde{w}^{i}\right) \mid i=\right.$ $\left.0, \ldots, N_{O}\right\}$ be the set of trackers used in the previous frame and $\left\{\left(P_{*}^{i}, w_{*}^{i}\right)^{t+1} \mid i=0, \ldots, N_{O}\right\}$, be the current best ones as defined in Equation 3.33, but for the set $\mathcal{P}_{i}^{t+1}$. The first candidate trackers for the current frame are given by the set $\mathcal{F}_{0}^{t}=\left\{\left(P^{i}, w^{i}\right) \mid i=1, \ldots, N_{O}\right\}$, where:

$$
\left(P^{i}, w^{i}\right)= \begin{cases}\left(P_{*}^{i}, w_{*}^{i}\right), & \text { if } w_{*}^{i}>\tau_{T} \tilde{w}^{i} \\ \left(\tilde{P}^{i}, \tilde{w}^{i}\right), & \text { otherwise }\end{cases}
$$

and $\tau_{T}$ is a given threshold for changing trackers. The final trackers are chosen as every $P^{j}$ that does not overlap any other $P^{k}$ by:

$$
\mathcal{F}_{1}^{t}=\left\{\left(P^{j}, w^{j}\right) \mid \forall\left(P^{k}, w^{k}\right) \in \mathcal{F}_{0}^{t}, j \neq k, d\left(r_{P}\left(P^{j}\right), r_{P}\left(P^{k}\right)\right)>\tau_{d_{D}}\right\}
$$

where $\tau_{d_{D}}$ represents a given distance threshold for different objects.

Overlapping trackers were not included because, when dealing with situations where both appearance and structure are ambiguous (e.g. symmetrical scenes), this method may end up associating multiple trackers with the same object. Therefore, this approach, which tries to find a configuration where each tracker is associated with a different object, is proposed. In other words, configurations where there is less overlapping between trackers of different objects are favored.

Let $\mathcal{M}=\{m\}$ be the set of indices of the objects whose trackers were not included in $\mathcal{F}_{1}^{t}$ and $\Omega_{P}=\left\{\omega^{m}=\left(\left|\mathcal{P}_{m}^{t+1}\right|, w_{*}^{m}, \mathcal{P}_{m}^{t+1}\right)\right\}$ be a set of triplets containing the cardinality and best temporal weight of each set of trackers $\mathcal{P}_{m}^{t+1}$. Let also $\Omega_{S}=\left(\omega^{m} \mid \omega^{m} \in \Omega_{P}\right)$ be a sequencing of $\Omega_{P}$ where the elements are ordered in increasing order of $\left|\mathcal{P}_{m}^{t+1}\right|$ and secondly by decreasing order of $w_{*}^{M}$ (lexicographical order). In other words, the $\omega^{m}$ are ordered by the cardinality of their tracker sets, but any pair $\left(\omega^{j}, \omega^{k}\right)$ that has the same cardinality is ordered by its respective weight.

The best tracker for each overlapping object $m$ is chosen by iteratively following the order given by $\Omega_{S}$. Therefore, the process starts by choosing the best trackers from the objects which have the smallest number of candidate trackers. This is done because, as the trackers are being chosen, the remaining free area (that is not covered by any tracker) is decreased. By processing objects with more trackers later, it is more likely that they will have candidates 
in the free area. The set of final trackers for the overlapping objects is obtained by:

$$
\mathcal{F}_{n+2}^{t}= \begin{cases}\mathcal{F}_{n+1}^{t} \cup\left(P_{k}^{m}, w_{k}^{m}\right), & \text { if }\left[\exists\left(P_{k}^{m}, w_{k}^{m}\right) \in \mathcal{P}_{m}^{t+1} \mid w_{k}^{m} \geq \tau_{O} w^{m}\right. \text { or } \\ & \left.d\left(r_{P}\left(P_{k}^{m}\right), r_{P}\left(P_{l}^{a}\right)\right) \geq \tau_{d_{D}}, \forall\left(P_{l}^{a}, w_{l}^{a}\right) \in \mathcal{F}_{n+1}^{t}\right] \\ \mathcal{F}_{n+1}^{t} \cup\left(P^{m}, w^{m}\right), & \text { otherwise }\end{cases}
$$

where $0 \leq n<|\mathcal{M}|$ represents the iteration index, $\left(P^{m}, w^{m}\right)$ is as defined in Equation 3.37, and $\tau_{O}$ is a given constant which represents a score weight threshold for changing trackers after overlapping. In other words, Equation 3.39 states that a tracker $P_{k}^{i}$ is chosen for object $m$ if it passes one of two tests: the first one is that it must not overlap any previously selected tracker for another object. The second test accepts overlapping trackers, but only if they have a high enough score. If no candidate is able to fulfill any of the requirements, then the first candidate obtained previously is chosen.

The final step consists in using the elements $\left(P^{i}, w^{i}\right) \in \mathcal{F}_{|\mathcal{M}|+1}^{t}$ to estimate the position of each object at time $t$, which is obtained by $r_{P}\left(P^{i}\right)$. The tracking update procedure that is applied at each frame is summarized in Algorithm 3.1. 
1 Algorithm update_tracking $\left(G^{M}, M_{A}, M_{C}, \mathcal{P}^{t-1}, \tau_{d_{D}}, \tau_{d_{S}}, \tau_{O}, \tau_{S}, \tau_{T}\right)$

Data: A model graph $G^{M}$, adjacency and candidate matrices $M_{A}$ and $M_{C}$, respectively, a set of sets of trackers $\mathcal{P}^{t-1}=\left\{\mathcal{P}_{i}^{t-1}\right\}$, where $\mathcal{P}_{i}^{t-1}=\left\{\left(P_{j}^{i}, w_{j}^{i}\right)\right\}$ and various thresholds $\tau$

Result: A set $\mathcal{P}^{t}$ with the updated trackers and another set $\mathcal{F}^{t}=\left\{F_{i} \mid F_{i} \in \mathcal{P}_{i}^{t}\right\}$ containing the tracking decision for each object $i$

2

3

4

5

6

7

8

Algorithm 3.1: Tracking update algorithm. 


\section{Chapter 4}

\section{Action recognition}

In this chapter, the proposed approach for performing action recognition will be explained. Action classification is applied on each person present in the scene independently. Therefore, in one single scene many actions may be performed at the same time. It is also assumed that the actions of each individual change over time. Figure 4.1 shows an overview of the action framework.

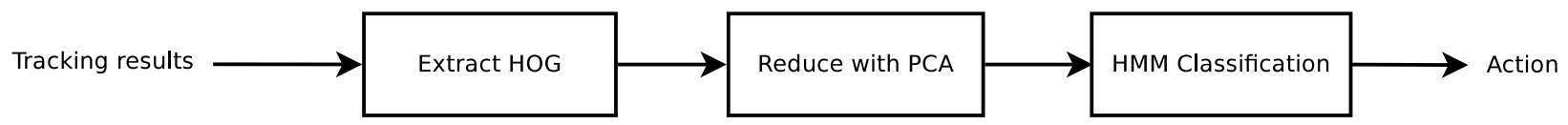

Figure 4.1: Overview of the action recognition framework.

The action classification is performed using Hidden Markov Models which operate over sequences of observations. The process starts by accumulating the results obtained from the tracking phase. Each person $i$ at instant $t$ is represented by a sliding window of tracking results $\tilde{\boldsymbol{o}}_{t}^{i}=\left(\tilde{o}_{t-\left\lfloor N_{W} / 2\right\rfloor}^{i}, \tilde{o}_{t-\left\lfloor N_{W} / 2\right\rfloor+1}^{i}, \ldots, \tilde{o}_{t}^{i}, \ldots, \tilde{o}_{t+\left\lfloor N_{W} / 2\right\rfloor-1}^{i}, \tilde{o}_{t+\left\lfloor N_{W} / 2\right\rfloor}^{i}\right)$, where $N_{W}$ is the length of the window. In order to classify the sequence, feature vectors are extracted from each $\tilde{o}_{t}^{i}$. This is accomplished by first computing the Histogram of Oriented Gradients (Section 4.1) and then reducing it using Principal Component Analysis (Section 4.2), yielding the new sequence of observations $\boldsymbol{o}_{t}^{i}$.

As usual, it is assumed that the set of possible actions is known beforehand. For each action $j$, a different HMM $Z_{j}$ is trained using annotated data. Since the training is supervised, each training instance consists of a sequence and an action label. The detailed training steps are explained in Section 4.3.3. After all HMMs are trained, the classification consists in finding which of the $Z_{j}$ best represents each observation $\boldsymbol{o}_{t}^{i}$ (Section 4.3.2). As evidenced by the definition of $\boldsymbol{o}_{t}^{i}$, we follow the conventional approach of building the sequence around the center. The main advantage is that in this way it is possible to provide a label while the action is being performed, as opposed to providing it at the end. On the other hand, it also implies that future data is required. Therefore, the action recognition must be delayed by 
$\left\lfloor N_{W} / 2\right\rfloor$ frames. However, as $N_{W}$ is usually small compared to the frame rate of the videos, the delay is not significant in practice.

\subsection{Histogram of Oriented Gradients (HOG)}

The Histogram of Gradients is a descriptor proposed by Dalal and Triggs (2005). It is used for representing the shape of an object, which is built by computing the image gradients and then populating a histogram in which each bin represents one gradient direction.

Computing the HOG consists in applying a series of standard methods in sequence over the initial image until obtaining the final histogram. In their paper, Dalal and Triggs (2005) provide a detailed coverage of many choices for each step and the observed results. For brevity, in this section only the actual method used in this work, which corresponds to the optimal configuration found by the authors, will be detailed. Figure 4.2 shows the main steps involved in computing the HOG.

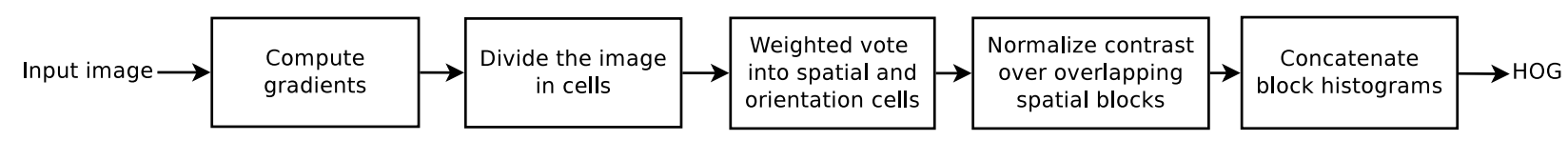

Figure 4.2: Overview of HOG chain. Adapted from (Dalal and Triggs (2005)).

The first step consists in computing the gradient of the image. This may be done by convolving the image with the simple centered kernels $\boldsymbol{K}_{\boldsymbol{H}}=[-1,0,1]$ and $\boldsymbol{K}_{\boldsymbol{V}}=[-1,0,1]^{T}$ for obtaining the horizontal and vertical gradients, respectively. The convolution of an image $I$ by a kernel matrix $\boldsymbol{K}$ with $N_{R}$ rows and $N_{C}$ columns is represented by $I_{C}=I * \boldsymbol{K}$ and can be computed by:

$$
I_{C}(x, y)=\sum_{r=-\left\lfloor N_{R} / 2\right\rfloor}^{\left\lfloor N_{R} / 2\right\rfloor} \sum_{c=-\left\lfloor N_{C} / 2\right\rfloor}^{\left\lfloor N_{C} / 2\right\rfloor} I(x+c, y+r) \boldsymbol{K}\left(\left\lfloor N_{C} / 2\right\rfloor+c,\left\lfloor N_{R} / 2\right\rfloor+r\right) \text {, }
$$

where the values inside the parenthesis indicate a position inside the image or kernel.

The convolution of $I$ by $\boldsymbol{K}_{\boldsymbol{H}}$ and $\boldsymbol{K}_{\boldsymbol{V}}$ yields two gradient images $I_{G X}$ and $I_{G Y}$, respectively. These images are then used to compute the gradient direction at each pixel:

$$
I_{G D}(x, y)=\arctan \left(\frac{I_{G Y}(x, y)}{I_{G X}(x, y)}\right)
$$

and the gradient magnitudes:

$$
I_{G M}(x, y)=\sqrt{I_{G X}(x, y)^{2}+I_{G Y}(x, y)^{2}} .
$$

The image is then divided in cells using a rectangular grid. Typically, each cell is an $8 \times 8$ 
square. Each pixel inside the cell then casts a vote for its gradient orientation, to create a cell histogram. The votes are weighted by $I_{G M}$, i.e. stronger gradients contribute more to the histogram. According to the authors, building a histogram with 9 bins using the unsigned gradients values (only considering the interval $\left[0^{\circ}, 180^{\circ}\right]$ ) provides the best results.

To account for contrast and illumination changes, the cells are grouped into larger rectangular blocks, which partially intersect each other. The histograms of each cell inside the block are then concatenated to form a larger vector. In this work, each block is a square containing $2 \times 2=4$ cells. The blocks share two cells with a neighbor block, i.e. the bottom two cells of a block are shared with its bottom neighbor and so on. Among the normalization choices, the most widely used and also employed here is the $L_{2}-H y s$ norm. This norm is computed by first computing the $L_{2}$ norm followed by a clipping which limits the maximum values to 0.2 . After this step, the resulting vector is once again normalized with the $L_{2}$ norm.

After normalizing all the blocks, the HOG descriptor is obtained by concatenating all the block histograms. One important fact to notice is that the HOG descriptor by itself is not invariant to shape and scale changes. Therefore, a fixed window size must be chosen beforehand to represent the object of interest. Since HOG is most widely applied for person detection, the window size is typically chosen as a vertical rectangle of $64 \times 128$ pixels. Due to the construction constraints, HOG descriptors are only comparable when extracted from windows of the same size. In order to overcome this restriction, the most commonly adopted approach is to resize the original detection to the chosen size before extracting its HOG.

As mentioned, each cell histogram is represented by a vector of length 9 . As each block is a square comprising 4 cells, then its corresponding histogram contains 36 bins. Each block can be viewed as a $16 \times 16$ square, where half of it intersects with its horizontal and vertical neighbors. Since the window size is $64 \times 128$ pixels, it can be seen that the window contains $7 \times 15=105$ blocks. Finally, by multiplying this value by the histogram length of each block, it can be seen that the resulting HOG vector has a high dimensionality of $105 \times 36=3780$. As it is usually intractable to classify data of this order of magnitude, we chose to reduce the descriptor length by using Principal Component Analysis.

\subsection{Principal Components Analysis (PCA)}

Essentially, PCA aims at applying an orthonormal transformation to multivariate data so that it becomes uncorrelated. The main point is that, if the data is uncorrelated, then it is possible to ignore (remove) one variable (axis) without affecting the variance of the others. In other words, by applying PCA it is possible to reduce the dimensionality of the data by discarding bases while minimizing the variance loss. This property is important because, according to Information Theory, the more uncertainty exists, the more information is available. Thus, keeping the data with as much variance as possible also minimizes the 
information loss.

The contents of this section are based on the publications of Callioli et al. (2007) and Shlens (2014). Let $\boldsymbol{O}=\left[\boldsymbol{o}_{1}, \boldsymbol{o}_{2}, \ldots, \boldsymbol{o}_{N}\right]^{T}$ be a data matrix where each $\boldsymbol{o}_{i}$ is one observation vector. It will be assumed that all the variables of $\boldsymbol{O}$ have zero mean. Let also $\boldsymbol{C}_{\boldsymbol{O}}=\frac{1}{N} \boldsymbol{O} \boldsymbol{O}^{T}$ be the covariance matrix of $\boldsymbol{O}$. The goal of PCA is to find a matrix $\boldsymbol{P}$ to transform the data as $\boldsymbol{Y}=\boldsymbol{P} \boldsymbol{O}$ such that the correlation matrix $\boldsymbol{C}_{\boldsymbol{Y}}=\frac{1}{N} \boldsymbol{Y} \boldsymbol{Y}^{T}$ is diagonal.

As shown in the paper by Shlens (2014), this problem corresponds to finding the eigenvectors of $\boldsymbol{C}_{\boldsymbol{O}}$. In fact, as also explained in the same material, another approach, and actually the most used in practice, is performing the Singular Value Decomposition to obtain the Principal Components. However, explaining the SVD is out of the scope of this thesis, so we will focus on giving an overview of how to obtain the eigenvectors of the covariance matrix.

The eigenvectors of a square matrix, such as $\boldsymbol{C}_{\boldsymbol{O}}$, can be found by solving:

$$
\begin{aligned}
\boldsymbol{C}_{\boldsymbol{O}} \boldsymbol{v} & =\lambda \boldsymbol{v} \\
\left(\boldsymbol{C}_{\boldsymbol{O}}-\lambda \boldsymbol{I}\right) \boldsymbol{v} & =0
\end{aligned}
$$

where $\boldsymbol{v}$ is an eigenvector of $\boldsymbol{C}_{\boldsymbol{O}}$ corresponding to the eigenvalue $\lambda$ and $\boldsymbol{I}$ is the identity matrix. From this formulation, it is clear that the eigenvalues are necessary to compute the eigenvectors. Eigenvalues can be obtained by solving the characteristic polynomial of $\boldsymbol{C}_{\boldsymbol{O}}$ :

$$
p(\lambda)=\operatorname{det}\left(\boldsymbol{C}_{\boldsymbol{O}}-\lambda \boldsymbol{I}\right)
$$

Each eigenvector will represent a base of the space transformation. Data reduction can be performed by simply transforming the data using only the bases with the highest variances, which minimizes the information loss.

\subsection{Hidden Markov Model (HMM)}

Hidden Markov Models are able to model the statistical properties of signals that present some temporal constraints. More specifically, it can be successfully used for problems that follow the Markov property, i.e. the state at time $t$ only depends on a finite set of states at previous time instants. The most widely studied situation assumes that the state at time $t$ only depends on the state at time $t-1$. In this case it is said that the HMM follows a Markov property of first order. In this chapter it is assumed that the HMMs are always of first order. The contents of this section are based on the publications of Duda et al. (2001) and Fink (2008).

In a typical Markov problem, the signal is represented by a sequence of observations 
at successive time instants. Let $\boldsymbol{o}=\left(o_{1}, o_{2}, \ldots, o_{T}\right)$ be such a sequence of length $T$. It is assumed that this sequence is generated by a discrete stochastic process, which includes a finite number of states and state transitions. More formally, a basic Markov model (without hidden states) is represented by a finite set of states

$$
\mathcal{S}=\left\{s_{1}, s_{2}, \ldots, s_{N_{S}}\right\}
$$

which will be referred to only by their indices, i.e. $i=s_{i}$. It also requires a matrix of state transition probabilities

$$
\boldsymbol{A}=\left\{a_{i j} \mid a_{i j}=\mathbb{P}\left(S_{t}=j \mid S_{t-1}=i\right)\right\} \text { such that } \sum_{j} a_{i j}=1 \text { for all } i
$$

and a vector of starting probabilities

$$
\boldsymbol{\pi}=\left(\pi_{i} \mid \pi_{i}=\mathbb{P}\left(S_{1}=i\right)\right)
$$

A Markov model can be interpreted as a complete directed graph where the arcs are labeled with transition probabilities. Figure 4.3 shows an example of a Markov model.

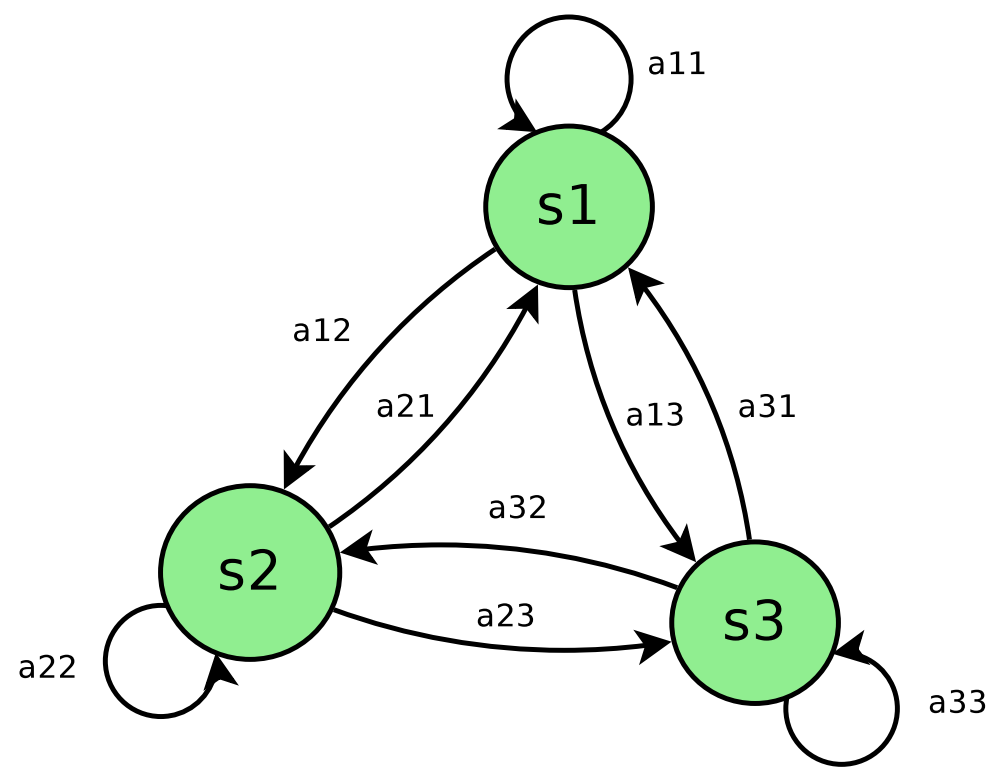

Figure 4.3: Example of a Markov model with three states. Each circle represents one state, and the directed edges the transitions from one state to another

In the basic model, it is assumed that the observation corresponds to the states themselves, i.e. each $o_{i}$ is directly mapped to a state $s_{i}$. However, in real situations it is often not possible to observe the states directly. For example, when modeling human speech, one might use each state to represent one phoneme and then follow one sequence of phonemes to produce a word. In practice, though, the phonemes cannot be perceived directly, only 
the sound they produce. Therefore, it is necessary to extend the basic model to account for the visible states (sounds) and the invisible ones (phonemes). By doing so, the HMM is obtained. In this approach, the basic Markov model discussed before corresponds to the hidden part, that cannot be directly observed. Each hidden state now emits a visible symbol (observation) $o_{i}$. This symbol is generated according to a distribution that can be either discrete (a finite set of possible observations) or continuous (modeled by a probability density function). In this project the emissions are obtained by continuous distributions given by a Gaussian mixture model. Hence, the emission probabilities are modeled by the vector:

$$
\boldsymbol{b}=\left(b_{j} \mid b_{j}=\sum_{m=1}^{N_{M}} c_{j m} \mathcal{N}\left(\boldsymbol{\mu}_{j m}, \boldsymbol{C}_{j m}\right)\right)
$$

where $c_{j m}$ is a normalization constraint such that

$$
\sum_{m=1}^{N_{M}} c_{j m}=1 \text { and } 0 \leq c_{j m} \leq 1, \text { for all }(j, m)
$$

The probability of a hidden state $j$ emitting a specific observation $o_{k}$ shall be represented by:

$$
b_{j}\left(o_{k}\right)=\mathbb{P}\left(o_{k} \mid S_{t}=j\right)=\sum_{m=1}^{N_{M}} c_{j m} \mathcal{N}\left(o_{k} \mid \boldsymbol{\mu}_{j m}, \boldsymbol{C}_{j m}\right)=\sum_{m=1}^{N_{M}} c_{j m} g_{j m}\left(o_{k}\right),
$$

where $\mathcal{N}\left(o_{k} \mid \boldsymbol{\mu}_{m}, \boldsymbol{C}_{m}\right)=g_{j m}\left(o_{k}\right)$ represents the probability of obtaining $o_{k}$ from a multivariate Gaussian (normal) distribution with mean vector $\boldsymbol{\mu}_{j m}$ and covariance matrix $\boldsymbol{C}_{j m}$. Figure 4.4 shows an example of a HMM.

When working with HMMs, there are usually three central problems to be considered: evaluation, decoding and learning. However, in the scope of this thesis, the decoding problem is not going to be studied and thus, will not be explained. On the other hand, another problem will be considered, which will be referred as the classification problem. A short description of each problem is provided below:

- Evaluation: This problem consists in, given a HMM, determining the probability that an observed sequence was generated from that model.

- Classification: In this problem, it will be assumed that a set of different HMMs is available and the interest is in finding which of those best represents a particular sequence.

- Learning: This problem is concerned to, given a set of observations and a coarse structure of the model (e.g. number of hidden states), finding the parameters that maximize the probability of the model generating those observations.

In the following sections, the three aforementioned problems will be further explained. 


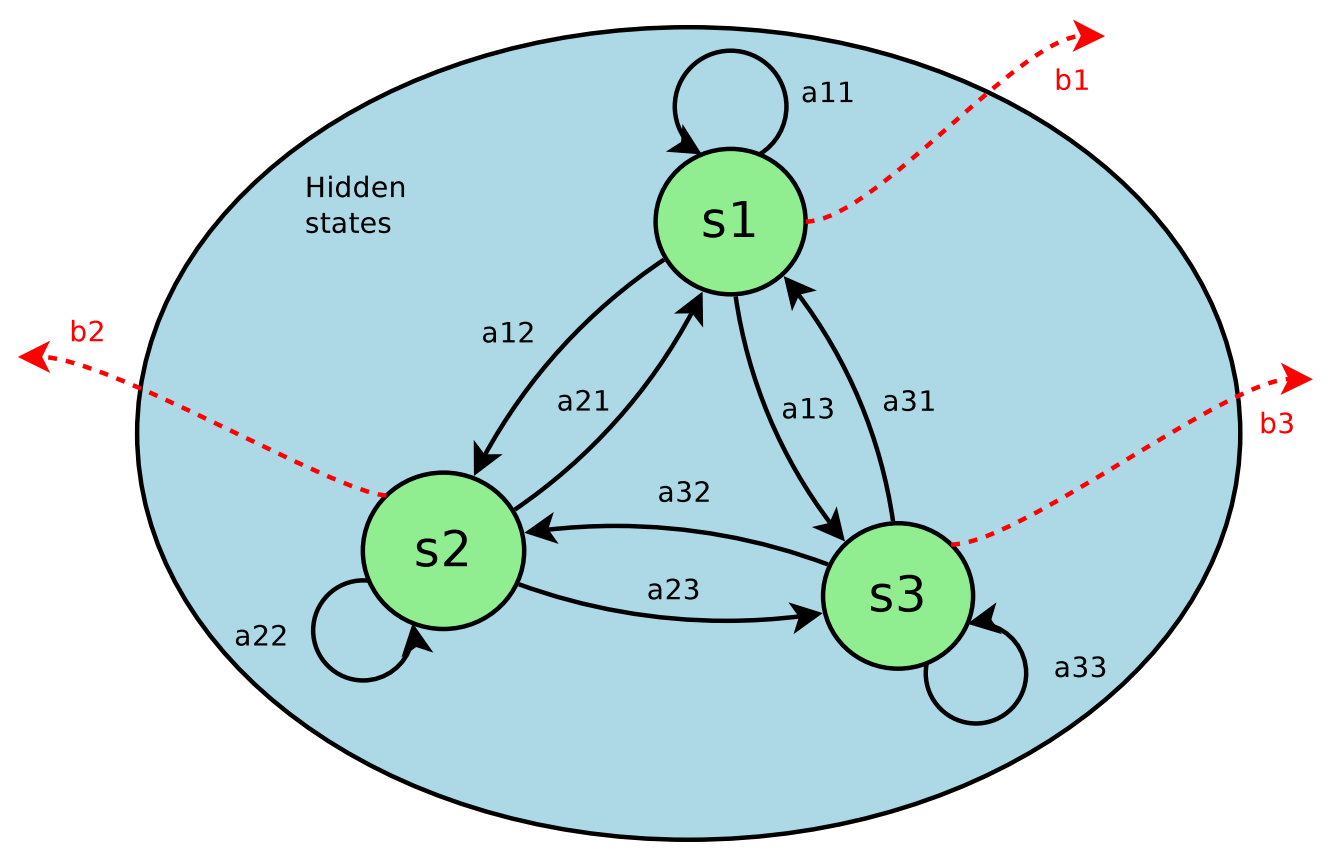

Figure 4.4: Example of a Hidden Markov model. The three hidden states (inside the ellipse) represent the unobservable properties of the data. Each hidden state has a probability of emitting a certain observable signal (red arrows).

\subsubsection{Evaluation}

Suppose that a sequence $\boldsymbol{o}=\left(o_{1}, o_{2}, \ldots, o_{T}\right)$ is given. Since each hidden state can only emit one observation at a time, then this sequence can only be generated by walking through a path of states $\boldsymbol{s}=\left(s_{1}, s_{2}, \ldots, s_{T}\right)$ with the same length $T$. If the path is known, then the production probability of a HMM $Z$ can be simply obtained by

$$
\mathbb{P}(\boldsymbol{o} \mid \boldsymbol{s}, Z)=\prod_{t=1}^{T} b_{t}\left(o_{t}\right)
$$

The probability that such a path $s$ is followed is computed by the product of the respective state transition probabilities. If we define $a_{0 i}=\pi_{i}$ and $s_{0}=0$, we can write:

$$
\mathbb{P}(\boldsymbol{s} \mid Z)=\pi_{1} \prod_{t=2}^{T} a_{t-1, t}=\prod_{t=1}^{T} a_{t-1, t} .
$$

By combining Equations 4.12 and 4.13, it is possible to compute the probability that the sequence $\boldsymbol{o}$ is generated by following the specific path $s$ by:

$$
\mathbb{P}(\boldsymbol{o}, \boldsymbol{s} \mid Z)=\mathbb{P}(\boldsymbol{o} \mid \boldsymbol{s}, Z) \mathbb{P}(\boldsymbol{s} \mid Z)=\prod_{t=1}^{T} a_{t-1, t} b_{t}\left(o_{t}\right)
$$

In order to compute the total production probability, it is necessary to accumulate over all the possible paths, which include all possible permutations of states in a sequence of 
length $T$. This can be expressed as:

$$
\mathbb{P}(\boldsymbol{o} \mid Z)=\sum_{\boldsymbol{s}} \mathbb{P}(\boldsymbol{o}, \boldsymbol{s} \mid Z)=\sum_{\boldsymbol{s}} \mathbb{P}(\boldsymbol{o} \mid \boldsymbol{s}, Z) \mathbb{P}(\boldsymbol{s} \mid Z)
$$

As there are $N_{S}^{T}$ possible paths, if this approach were implemented as an algorithm, its complexity would be $O\left(T N_{S}^{T}\right)$. For most problems, an exponential complexity is not tractable and thus, cannot be used in practice. Fortunately, a much more efficient approach can be derived by exploring the Markov property.

As it can be seen in Equation 4.14, each term only depends on the current and previous states. It is not necessary to know at which time point each state is visited or in which order. Therefore, the computations for each state can be done in parallel throughout the time line. To this aim, a recurrence relation that leads to the well-known forward algorithm can be used. The first step is to define the forward variables $\alpha_{t}(i)$, that correspond to the probability that the first part of the observation until $O_{t}$ is evaluated and the state $i$ is reached at time $t$ :

$$
\alpha_{t}(i)=\mathbb{P}\left(O_{1}, O_{2}, \ldots, O_{t}, S_{t}=i \mid Z\right)
$$

At the beginning, the probability $\alpha_{1}(i)$ of generating the observation $O_{1}$ at state $i$ is given by the starting probability $\pi_{i}$ and the emission probability $b_{i}\left(O_{1}\right)$ :

$$
\alpha_{1}(i)=\pi_{i} b_{i}\left(O_{1}\right)
$$

Afterwards, the new probabilities for the next time instant $t+1$ can be obtained by combining the results computed at the last time instant $t$. For each state $j$ at time $t+1$, it is necessary to consider all possible paths that come from all other states $i$ at time $t$. Additionally, the new observation $O_{t+1}$ must be generated from $s_{j}$. Therefore, the forward variables can be updated by:

$$
\alpha_{t+1}(j)=\left(\sum_{i} \alpha_{t}(i) a_{i j}\right) b_{j}\left(O_{t+1}\right) .
$$

By computing until time $T, N_{S}$ results will be obtained, one for each state. As the complexity of computing each path is $O\left(T N_{S}\right)$, the complexity of the forward algorithm is $O\left(T N_{S}^{2}\right)$. Finally, the probability of model $Z$ producing the sequence $\boldsymbol{o}$ is:

$$
\mathbb{P}(\boldsymbol{o} \mid Z)=\sum_{i=1}^{N} \alpha_{T}(i)
$$

The pseudocode for performing the Forward procedure is shown in Algorithm 4.1. The production probability can be directly obtained by summing up the respective values of the 
resulting matrix, as presented in Algorithm 4.2.

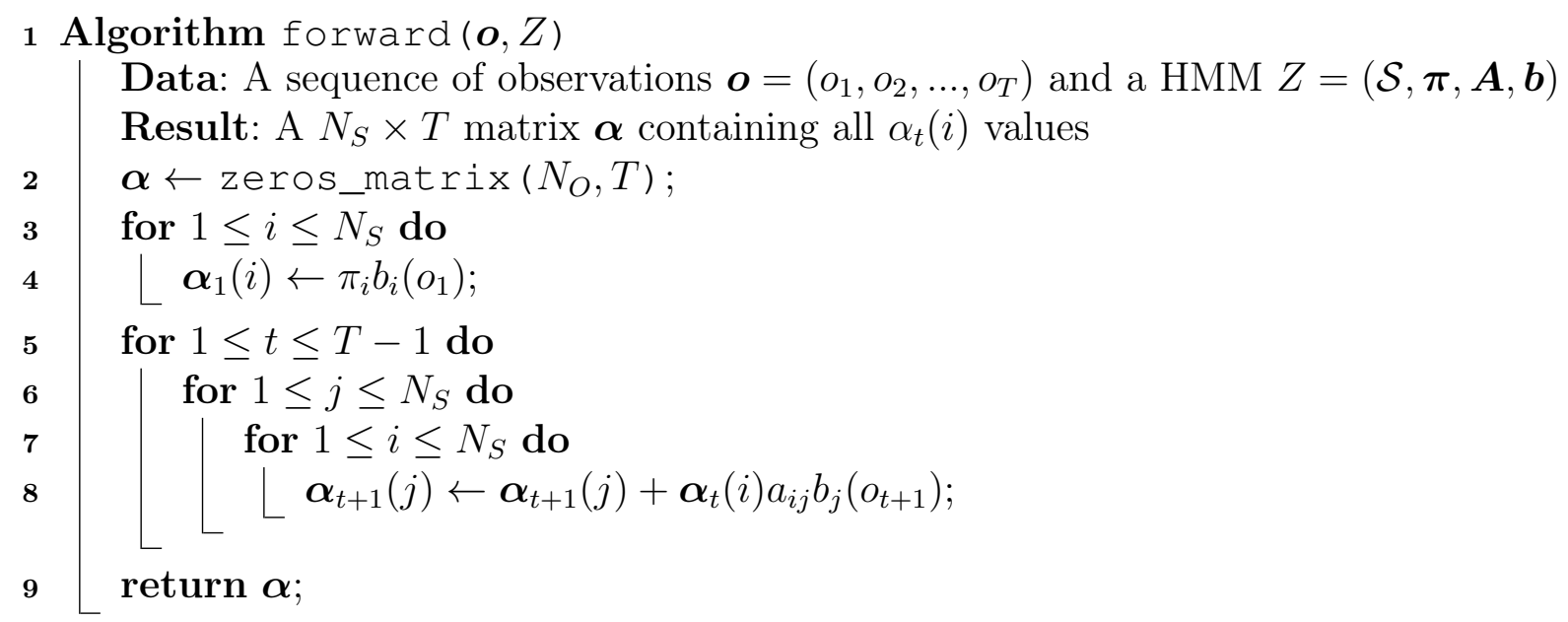

Algorithm 4.1: Forward algorithm.

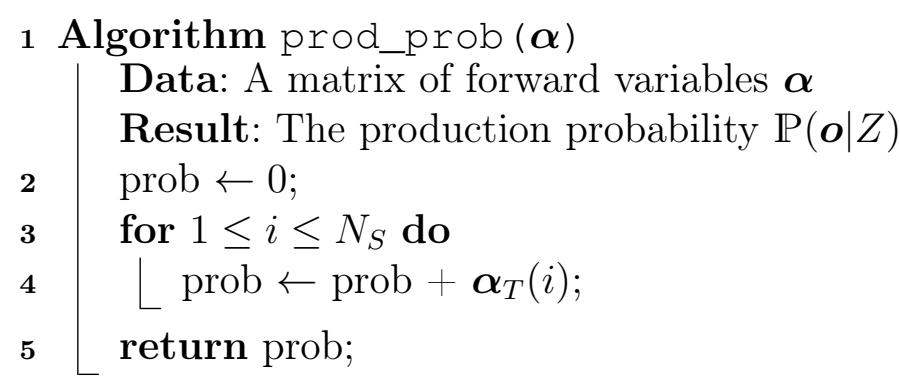

Algorithm 4.2: Production probability algorithm.

\subsubsection{Classification}

In some situations, several HMMs $Z_{i}$, each representing a different class $\omega_{i}$ are available. In this case, an observation $\boldsymbol{o}$ may be classified by finding the model $Z_{j}$ that maximizes the posterior probability $Z_{j}=\arg \max _{i} \mathbb{P}\left(Z_{i} \mid \boldsymbol{o}\right)$. By using the Bayes' theorem, this expression may be rewritten as:

$$
Z_{j}=\underset{i}{\arg \max } \frac{\mathbb{P}\left(\boldsymbol{o} \mid Z_{i}\right) \mathbb{P}\left(Z_{i}\right)}{\mathbb{P}(\boldsymbol{o})} .
$$

Since the denominator is independent of $Z_{i}$, it can be ignored for the classification purpose, yielding:

$$
Z_{j}=\underset{i}{\arg \max } \mathbb{P}\left(\boldsymbol{o} \mid Z_{i}\right) \mathbb{P}\left(Z_{i}\right) .
$$

Notice that the first term is the production probability explained in Section 4.3.1, which implies that the classification problem depends on the evaluation problem. Besides that, it is also necessary to know $\mathbb{P}\left(Z_{i}\right)$. This knowledge may come from an expert or from observation of the data, if available. For simplicity, however, this term is often ignored, and classification is done based solely on the evaluation result. In practice, this corresponds to setting $\mathbb{P}\left(Z_{i}\right)=$ $1 / N$ for all $0<i \leq N$. 


\subsubsection{Learning}

A good model represents the properties of the data as precisely as possible. In order to do so, the free parameters of the model can be improved by observing the data. In the case of HMMs, the free parameters are the initial probabilities $\boldsymbol{\pi}$, the transition probabilities $\boldsymbol{A}$ and the emission probabilities $\boldsymbol{b}$. The values of each of these parameters provides an answer to the following question: out of the total outputs one state can provide, how many of them are expected to follow this specific path? Using mathematical terms, this corresponds to finding the optimized parameters $\hat{a}_{i j}$ and $\hat{b}_{i}\left(o_{k}\right)$ defined as:

$$
\begin{gathered}
\hat{a}_{i j}=\frac{\text { expected number of transitions from } i \text { to } j}{\text { expected number of transitions out of state } i}, \\
\hat{b}_{i}\left(o_{k}\right)=\frac{\text { expected number of emissions } o_{k} \text { from state } i}{\text { total number of emissions from state } i} .
\end{gathered}
$$

When using continuous emission models, the second step actually corresponds to finding the parameters of the probability density functions that best model the observations.

Two fundamentally different methods exist for training: the Baum-Welch and the Viterbi algorithms (more details in the book of Fink (2008)). The former can be seen as the Expectation Maximization algorithm applied to HMMs. The Baum-Welch algorithm evaluates all possible paths and thus, find parameters that optimize the model for all possible state configurations for the given observations. The Viterbi algorithm, on the other hand, first try to estimate the most likely path each observation will take and then optimize the parameters only for the obtained path. The Viterbi approach may be more efficient but it does not always provide the best global configuration.

In this work, we will use the Baum-Welch algorithm. One of the main reasons for this choice is that the Baum-Welch results can be used to analytically optimize the parameters of the continuous emission models, which is not possible when using the Viterbi approach.

\section{Forward-Backward Algorithm}

In order to optimize the parameters of the model, it is necessary to know what is the probability $\mathbb{P}\left(S_{t}=i \mid \boldsymbol{o}, Z\right)$ of being in a state $i$ at a given instant $t$. This can be done by using the Forward-Backward algorithm. Very similarly to the forward case, but in the opposing sense, backward variables are defined to represent the probability of generating the remaining sequence $\left(O_{t+1}, O_{t+2}, \ldots, O_{T}\right)$ starting from state $i$ :

$$
\beta_{t}(i)=\mathbb{P}\left(O_{t+1}, O_{t+2}, \ldots, O_{T} \mid S_{t}=i, Z\right)
$$


As in the forward approach, $\beta_{t}(i)$ can be computed by using a recurrence relation of the form:

$$
\beta_{t}(i)= \begin{cases}1, & \text { if } t=T \\ \sum_{j} a_{i j} b_{j}\left(O_{t+1}\right) \beta_{t+1}(j), & \text { otherwise. }\end{cases}
$$

Having both the forward and backward variables, it is possible to compute the desired probability. First, by applying the rule from Equation 3.5, we have:

$$
\mathbb{P}\left(S_{t}=i \mid \boldsymbol{o}, Z\right)=\frac{\mathbb{P}\left(S_{t}=i, \boldsymbol{o} \mid Z\right)}{\mathbb{P}(\boldsymbol{o} \mid Z)}
$$

The denominator is the production probability obtained by solving the evaluation problem. The numerator can be rewritten using the forward and backward variables:

$$
\begin{aligned}
\mathbb{P}\left(S_{t}=i, \boldsymbol{o} \mid Z\right) & =\mathbb{P}\left(O_{1}, O_{2}, \ldots, O_{t}, S_{t}=i \mid Z\right) \mathbb{P}\left(O_{t+1}, O_{t+2}, \ldots, O_{T} \mid S_{t}=i, Z\right) \\
& =\alpha_{t}(i) \beta_{t}(i)
\end{aligned}
$$

By replacing this in Equation 4.26, we obtain:

$$
\gamma_{t}(i)=\mathbb{P}\left(S_{t}=i \mid \boldsymbol{o}, Z\right)=\frac{\alpha_{t}(i) \beta_{t}(i)}{\mathbb{P}(\boldsymbol{o} \mid Z)}
$$

The value $\gamma_{t}(i)$ represents the probability of being in state $i$ at instant $t$. Analogously, it also represents the expected number of transitions out of state $i$ at the next time step. A pseudo code of the Backward procedure is provided in Algorithm 4.3. As it can be seen, the algorithm is just a reversed version of the Forward procedure. By combining these results with those obtained from the Forward step (Algorithm 4.1), the values of $\gamma_{t}(i)$ can be easily computed.

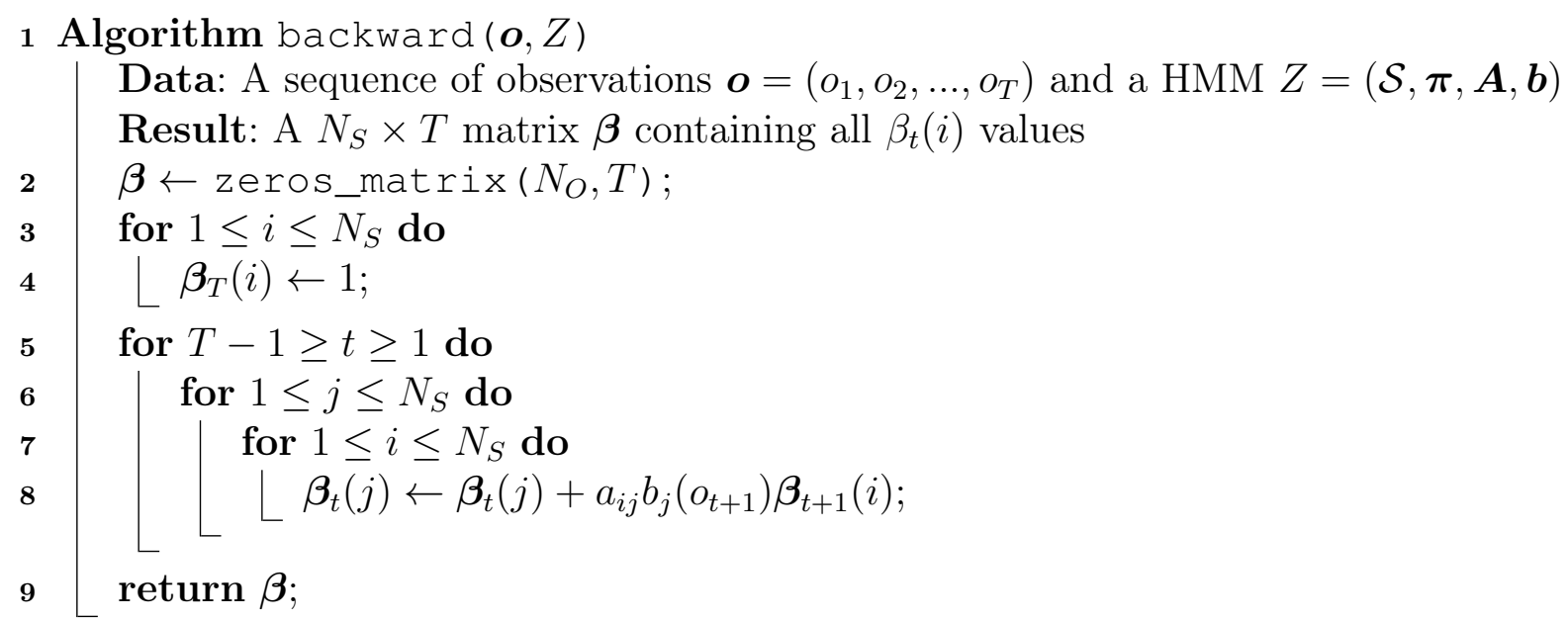

Algorithm 4.3: Backward algorithm. 


\section{Baum-Welch Algorithm}

The Baum-Welch algorithm optimizes the model parameters by using the production probability $\mathbb{P}(\boldsymbol{o} \mid Z)$. This algorithm guarantees that the new model $\hat{Z}$ produces the observations with equal or higher probability:

$$
\mathbb{P}(\boldsymbol{o} \mid \hat{Z}) \geq \mathbb{P}(\boldsymbol{o} \mid Z)
$$

Notice that this method may not provide the best possible fit, as it is bounded to stop at a local maximum. One simple way to overcome this problem is to run several training instances and choose the best one.

The Baum-Welch represents a variant of the Expectation Maximization algorithm. Besides representing the probability $\gamma_{t}(i)=\mathbb{P}\left(S_{t}=i \mid \boldsymbol{o}, Z\right)$ for a state in general, it is also interesting to represent the same probability but restricted to a specific transition from state $i$ to $j$ as follows:

$$
\begin{aligned}
\gamma_{t}(i, j) & =\mathbb{P}\left(S_{t}=i, S_{t+1}=j \mid \boldsymbol{o}, Z\right) \\
& =\frac{\mathbb{P}\left(S_{t}=i, S_{t+1}=j, \boldsymbol{o} \mid Z\right)}{\mathbb{P}(\boldsymbol{o} \mid Z)} \\
& =\frac{\alpha_{t}(i) a_{i j} b_{j}\left(o_{t+1}\right) \beta_{t+1}(j)}{\mathbb{P}(\boldsymbol{o} \mid Z)}
\end{aligned}
$$

Notice that this value represents the overall probability of going through transition $(i, j)$ at instant $t$. By computing $\gamma_{t}(i, j)$ for each pair $(i, j)$ over all time instants $t$ and averaging by the probability of leaving state $i$, the new estimates for the transition probabilities are obtained:

$$
\hat{a}_{i j}=\frac{\sum_{t=1}^{T-1} \mathbb{P}\left(S_{t}=i, S_{t+1}=j \mid \boldsymbol{o}, Z\right)}{\sum_{t=1}^{T-1} \mathbb{P}\left(S_{t}=i \mid \boldsymbol{o}, Z\right)}=\frac{\sum_{t=1}^{T-1} \gamma_{t}(i, j)}{\sum_{t=1}^{T-1} \gamma_{t}(i)} .
$$

The probability of leaving each state at the first time instant can be used as a starting probability:

$$
\hat{\pi}_{i}=\mathbb{P}\left(S_{1}=i \mid \boldsymbol{o}, Z\right)
$$

When using discrete emission probabilities, the new values can also be estimated analogously:

$$
\hat{b}_{j}\left(o_{k}\right)=\frac{\sum_{t=1}^{T-1} \mathbb{P}\left(S_{t}=j, O_{t}=o_{k} \mid \boldsymbol{o}, Z\right)}{\sum_{t=1}^{T} \mathbb{P}\left(S_{t}=j \mid \boldsymbol{o}, Z\right)}=\frac{\sum_{t: O_{t}=o_{k}} \mathrm{P}\left(S_{t}=j \mid \boldsymbol{o}, Z\right)}{\sum_{t=1}^{T} \mathbb{P}\left(S_{t}=j \mid \boldsymbol{o}, Z\right)}=\frac{\sum_{t: O_{t}=o_{k}} \gamma_{t}(j)}{\sum_{t=1}^{T} \gamma_{t}(j)} .
$$

Notice that in the numerator of the equation only the instants $t$ where $o_{k}$ was observed are considered.

However, when using continuous emission models, the parameters of the mixture models 
must be computed instead. This can be done by following a similar procedure to that used for discrete models. In order to do so, we observe that the parameters $c_{j k}$ of the mixture models merely represent the expected number of times the $k$-th mixture will be used when generating the emission for state $j$. Let $\xi_{t}(j, k)$ represent the probability of using mixture $k$ in state $j$ at instant $t$, defined as follows:

$$
\begin{aligned}
\xi_{t}(j, k) & =\mathbb{P}\left(S_{t}=j, M_{t}=k \mid \boldsymbol{o}, Z\right) \\
& =\frac{\mathbb{P}\left(S_{t}=j, M_{t}=k, \boldsymbol{o} \mid Z\right)}{\mathbb{P}(\boldsymbol{o}, Z)} \\
& =\frac{\sum_{i=1}^{N} \mathbb{P}\left(S_{t-1}=i, S_{t}=j, M_{t}=k, \boldsymbol{o} \mid Z\right)}{\mathbb{P}(\boldsymbol{o}, Z)} \\
& =\frac{\sum_{i=1}^{N} \alpha_{t-1}(i) a_{i j} c_{j k} g_{j k}\left(o_{t}\right) \beta_{t}(j)}{\mathbb{P}(\boldsymbol{o}, Z)} .
\end{aligned}
$$

Once again, by using the same procedure as in Equation 4.31, the new mixture weights can be obtained by:

$$
\hat{c}_{j k}=\frac{\sum_{t=1}^{T} \mathbb{P}\left(S_{t}=j, M_{t}=k \mid \boldsymbol{o}, Z\right)}{\sum_{t=1}^{T} \mathbb{P}\left(S_{t}=j \mid \boldsymbol{o}, Z\right)}=\frac{\sum_{t=1}^{T} \xi_{t}(j, k)}{\sum_{t=1}^{T} \gamma_{t}(j)} .
$$

By knowing the probability of each mixture being selected, it is now possible to update the parameters of each one of them. Since a Gaussian mixture model is being used, the parameters to be estimated are the mean vector and the covariance matrix. They can both be computed by using the classical formulas for statistical mean and covariance, weighted by the probability of the mixture being chosen as follows:

$$
\begin{aligned}
\hat{\boldsymbol{\mu}}_{j k} & =\frac{\sum_{t=1}^{T} \mathbb{P}\left(S_{t}=j, M_{t}=k \mid \boldsymbol{o}, Z\right) o_{t}}{\sum_{t=1}^{T} \mathbb{P}\left(S_{t}=j, M_{t}=k \mid \boldsymbol{o}, Z\right)}=\frac{\sum_{t=1}^{T} \xi_{t}(j, k) o_{t}}{\sum_{t=1}^{T} \xi_{t}(j, k)} . \\
\hat{\boldsymbol{C}}_{j k} & =\frac{\sum_{t=1}^{T} \mathbb{P}\left(S_{t}=j, M_{t}=k \mid \boldsymbol{o}, Z\right)\left(o_{t}-\hat{\boldsymbol{\mu}}_{j k}\right)\left(o_{t}-\hat{\boldsymbol{\mu}}_{j k}\right)^{T}}{\sum_{t=1}^{T} \mathbb{P}\left(S_{t}=j, M_{t}=k \mid \boldsymbol{o}, Z\right)} \\
& =\frac{\sum_{t=1}^{T} \xi_{t}(j, k)\left(o_{t}-\hat{\boldsymbol{\mu}}_{j k}\right)\left(o_{t}-\hat{\boldsymbol{\mu}}_{j k}\right)^{T}}{\sum_{t=1}^{T} \xi_{t}(j, k)} .
\end{aligned}
$$

By considering that the variance of a set of data $\boldsymbol{X}$ can be rewritten as:

$$
\operatorname{Var}[\boldsymbol{X}]=\mathrm{E}\left[\boldsymbol{X} \boldsymbol{X}^{T}\right]-\mathrm{E}[\boldsymbol{X}] \mathrm{E}[\boldsymbol{X}]^{T}
$$


Equation 4.37 can be rewritten as:

$$
\begin{aligned}
\hat{\boldsymbol{C}}_{j k} & =\frac{\sum_{t=1}^{T} \mathbb{P}\left(S_{t}=j, M_{t}=k \mid \boldsymbol{o}, Z\right) o_{t} o_{t}^{T}}{\sum_{t=1}^{T} \mathbb{P}\left(S_{t}=j, M_{t}=k \mid \boldsymbol{o}, Z\right)}-\hat{\boldsymbol{\mu}}_{j k} \hat{\boldsymbol{\mu}}_{j k}^{T} \\
& =\frac{\sum_{t=1}^{T} \xi_{t}(j, k) o_{t} o_{t}^{T}}{\sum_{t=1}^{T} \xi_{t}(j, k)}-\hat{\boldsymbol{\mu}}_{j k} \hat{\boldsymbol{\mu}}_{j k}^{T} .
\end{aligned}
$$

The advantage of this formulation is that each side of the difference can be computed in parallel in a single pass through the data. A pseudo code of the Baum-Welch procedure can be seen in Algorithm 4.4. 
1 Algorithm baum-welch (o, Z, stop_thresh, max_iter $)$

Data: A sequence of observations $\boldsymbol{o}=\left(o_{1}, o_{2}, \ldots, o_{T}\right)$, an initial HMM

$Z=(\mathcal{S}, \boldsymbol{\pi}, \boldsymbol{A}, \boldsymbol{b})$, a stopping threshold stop_thresh and an iteration threshold max_iter

Result: The updated HMM $\hat{Z}$ count $\leftarrow 0$; diff $\leftarrow \infty$;

Algorithm 4.4: Baum-Welch algorithm. 


\section{Chapter 5}

\section{Results}

In this chapter, the results both for the tracking and the action recognition modules will be presented and discussed. For each module, the datasets used for training and testing are explained. Afterwards, we present how the parameters for each method were computed based on training data. Finally, experimental results are shown.

\subsection{Multi-object tracking}

The software was developed in Python with the OpenCV library ${ }^{1}$. As explained in Section 3.2, each object is individually tracked using particle filters with color histograms as proposed by Pérez et al. (2002).

\subsubsection{Datasets}

The two datasets used for testing the tracking framework are described below.

\section{Youtube table tennis}

This dataset is composed of 6 videos containing 6737 frames in total. All the videos are of doubles matches of competitive table tennis collected from Youtube. Figure 5.1 shows some sample frames from this dataset. The videos were edited to remove unrelated scenes (e.g. preparation stage, crowd) and then manually annotated with bounding boxes for training and groundtruth. The videos are encoded at resolutions varying from $640 \times 360$ to $854 \times 480$ at 30FPS.

\section{ACASVA}

We selected three videos from the ACASVA (De Campos et al. (2011)) dataset of badminton doubles matches from the Olympic games in London 2012. As in the table tennis

\footnotetext{
${ }^{1}$ http://opencv.org/
} 

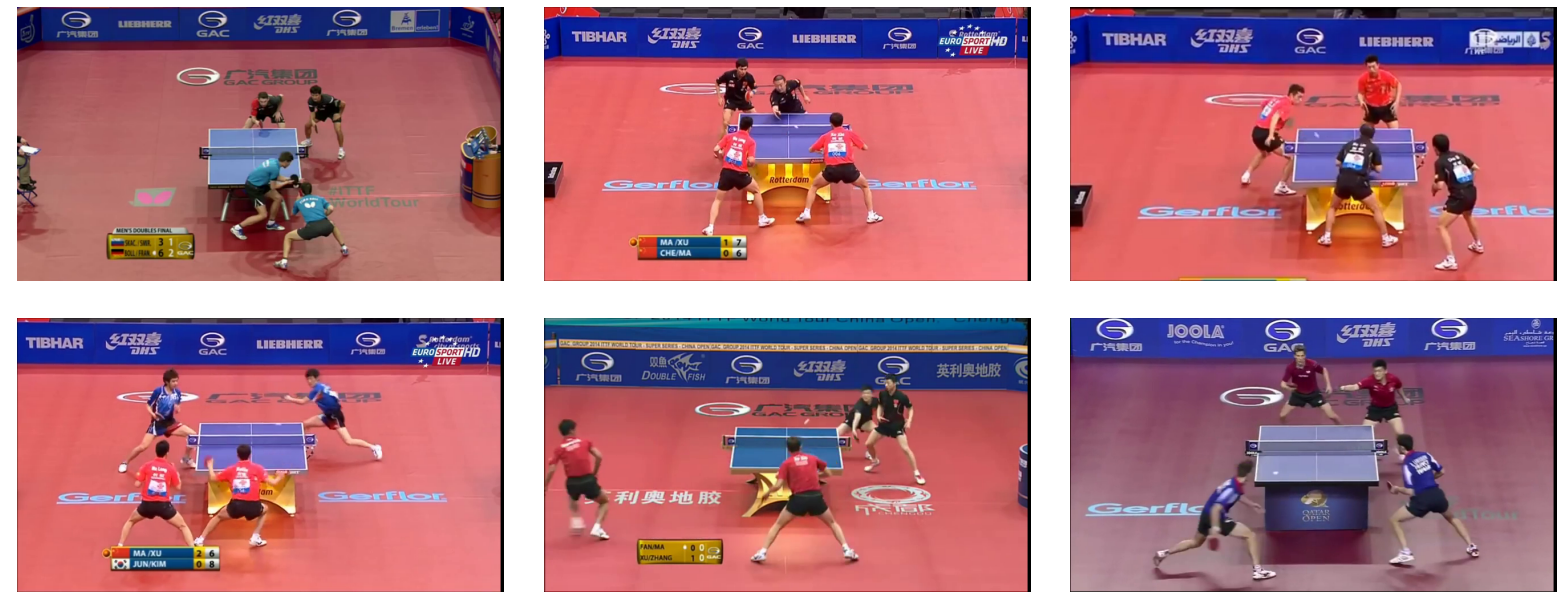

Figure 5.1: Sample frames from the Youtube table tennis dataset.

dataset, the videos were edited to remove parts that do not show the game itself and annotations were created manually to be used as groundtruth. The resulting videos were encoded at $854 \times 480$ at $30 \mathrm{FPS}$ and contained 5766 frames. Figure 5.2 displays some sample frames from this dataset.
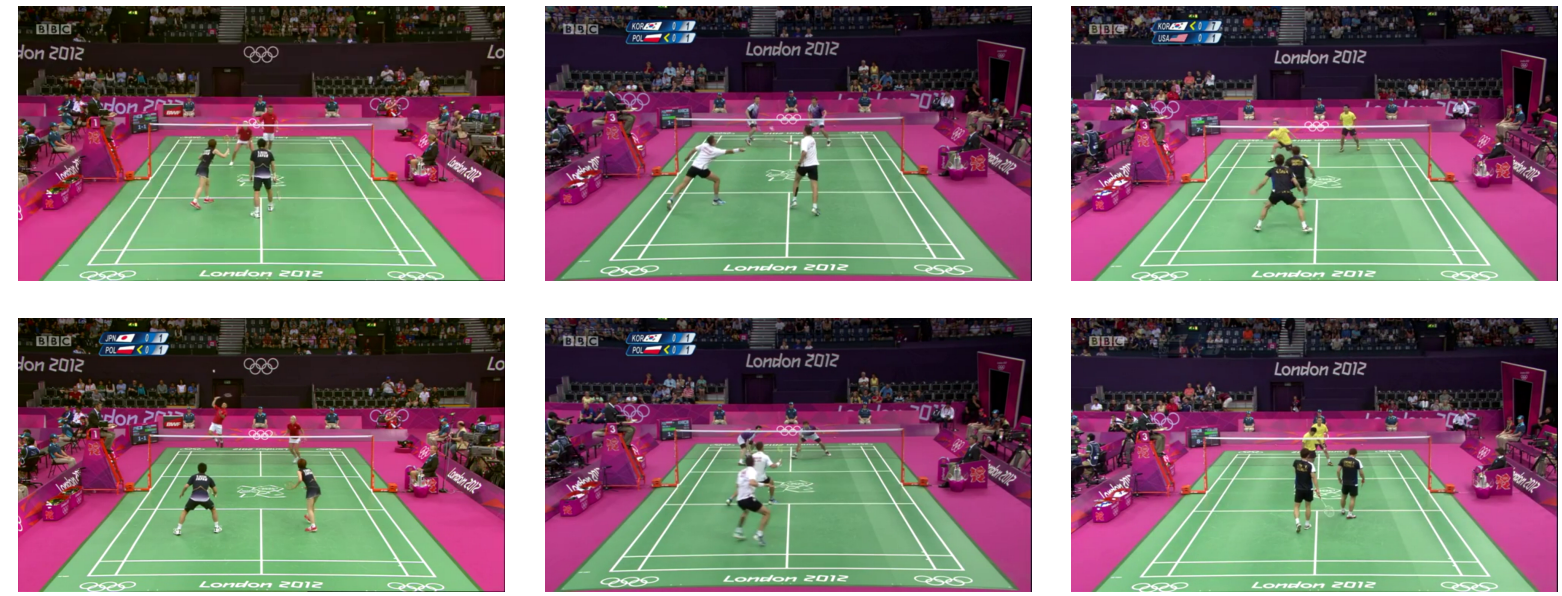

Figure 5.2: Sample frames from the ACASVA badminton dataset.

\subsubsection{Evaluation measurements}

The tracking results are evaluated using five measurements for each frame. First, let $N_{O}$ be the number of expected objects and $N_{F}$ the number of frames of the video. Let also $G B_{j}^{i}$ and $E B_{j}^{i}$ be the sets of points (pixels) inside the groundtruth and estimated bounding boxes, respectively, of the object $j$ in frame $i$ and $c(B)$ represent the centroid of a bounding box $B$. All the measurements are computed for every frame and then averaged by dividing their sum by $N_{F} N_{O}$. 
The first measurement is the center error (CERR) used in Adam et al. (2006), which consists of the Euclidean distance between the centroids of the bounding boxes, defined as

$$
\mathrm{CERR}_{i}=\sum_{j=1}^{N_{O}} d\left(c\left(G B_{j}^{i}\right), c\left(E B_{j}^{i}\right)\right) .
$$

The second measurement is the center displacement (CDIS), that evaluates the stability of the tracking. This is done by computing the distances between the center of each corresponding bounding box in two consecutive frames:

$$
\mathrm{CDIS}_{i}=\sum_{j=1}^{N_{O}} d\left(c\left(E B_{j}^{i}\right), c\left(E B_{j}^{i-1}\right)\right)
$$

The third measurement is the hit detection ratio (HITR). As proposed by Su et al. (2014), a detection is considered successful when $I\left(c\left(G B_{j}^{i}\right), E B_{j}^{i}\right)=1$, where $I(\cdot)$ is an indicative function that is equal to one when $c\left(G B_{j}^{i}\right) \in E B_{j}^{i}$. Therefore, the measurement is obtained by

$$
\mathrm{HITR}_{i}=\sum_{j=1}^{N_{O}} I\left(c\left(G B_{j}^{i}\right), E B_{j}^{i}\right) .
$$

The fourth measurement is the hit team ratio (HITT). This is a relaxed version of the HITR, where we consider that the tracking is correct even if the bounding boxes for the two players of the same team are swapped. Therefore, this measurement is only used for evaluating tracking for the sports datasets. The reason for using this measurement is that the appearance of players of the same team are very similar, and even for a human observer it is not trivial to identify each player correctly after situations of occlusion or camera cut. More formally, this measurement is computed by the following procedure. Consider the situation for the pair of players 1 and 2 of the same team. First we find the correspondence with the highest intersection: $\left(j^{*}, k^{*}\right)=\arg \max _{j, k=1,2}\left(G B_{j}^{i} \cap E B_{k}^{i}\right)$. If $j^{*} \neq k^{*}$ then we swap $E B_{1}^{i}$ and $E B_{2}^{i}$. The same procedure is applied for the other team. After correcting the bounding boxes, HITT is computed in the same way as HITR.

The fifth one is the object intersection ratio (OBJI) which measures the amount of intersection between the bounding boxes of the groundtruth and the result for the same object. This measurement is similar to the success plot used in the benchmark by Wu et al. (2015). More formally, it is defined as:

$$
\mathrm{OBJI}_{i}=\frac{\sum_{j=1}^{N_{O}}\left|G B_{j}^{i} \cap E B_{j}^{i}\right|}{\max \left\{\left|G B_{j}^{i}\right|,\left|E B_{j}^{i}\right|\right\}}
$$




\subsubsection{Estimation of parameters}

We separated the Youtube videos into two sets, one for parameter estimation and another for evaluation. The set for parameter estimation was composed of one video containing 2461 frames. This video contained a longer table tennis match containing all the expected challenging situation including overlapping between players of the same team and camera cuts. The model graph was learned using annotations available for all the other 5 videos.

The graph parameters were chosen by running tests on the evaluation video. We have to estimate three parameters: the feature weight $\rho_{F}$, the old weight factor $\rho_{T}$ and the threshold for removing trackers $\tau_{S}$. The parameters were estimated by keeping all of them fixed, except one. The fixed parameters were empirically chosen beforehand and their values are shown in Table 5.1. We chose to evaluate the results using the CERR measure, which is one of the most commonly adopted in tracking works. For reference, we ran the raw particle filter tracking on this video and the observed error was $\mathrm{CERR}=138$.

Table 5.1: Initial fixed parameters used for estimating the new ones.

\begin{tabular}{ll}
\hline feature weight & $\rho_{F}=0.5$ \\
old temporal weight factor & $\rho_{T}=0.75$ \\
score threshold for removing trackers & $\tau_{S}=0.25$ \\
\hline
\end{tabular}

The first parameter tested was the feature weight $\rho_{F}$. As can be seen in Figure 5.3, if the color model is totally ignored, the performance is very poor. However, the best results are obtained when the feature weight is kept low, showing that the structure is very important in this kind of video. Following the observed results, we chose to work with $\rho_{F}=0.4$.

The analysis of the old weight factor $\rho_{T}$ in Figure 5.4 shows that changing it does not significantly affect the results, except when its value is high. Indeed, when a high factor is used, the situation is the same as tracking with a single tracker, since no candidate will be able to have a higher score than older trackers. Thus, the observed result is similar to using raw particle filters. However, by analyzing the center displacement measure, it is clear that higher values increase the stability of the results. In that sense, this parameter is set as $\rho_{T}=0.75$, which keeps the error low while providing good stability.

Finally, from Figure 5.5 we observe that by using lower threshold values $\tau_{S}$ for removing trackers, the results are better. This happens because higher values may end up removing correct trackers in some cases, up to the point where only one tracker is kept, which is also similar to the raw particle filter approach. However, it is also important to notice that, by keeping more trackers, the running time is also impacted. As shown in the same Figure 5.5, when using no threshold, the performance is more than two times slower than when using a high value. Therefore, a good choice of parameter must take into consideration both the accuracy and running time. Hence, the value chosen for this parameter was $\tau_{S}=0.25$. 


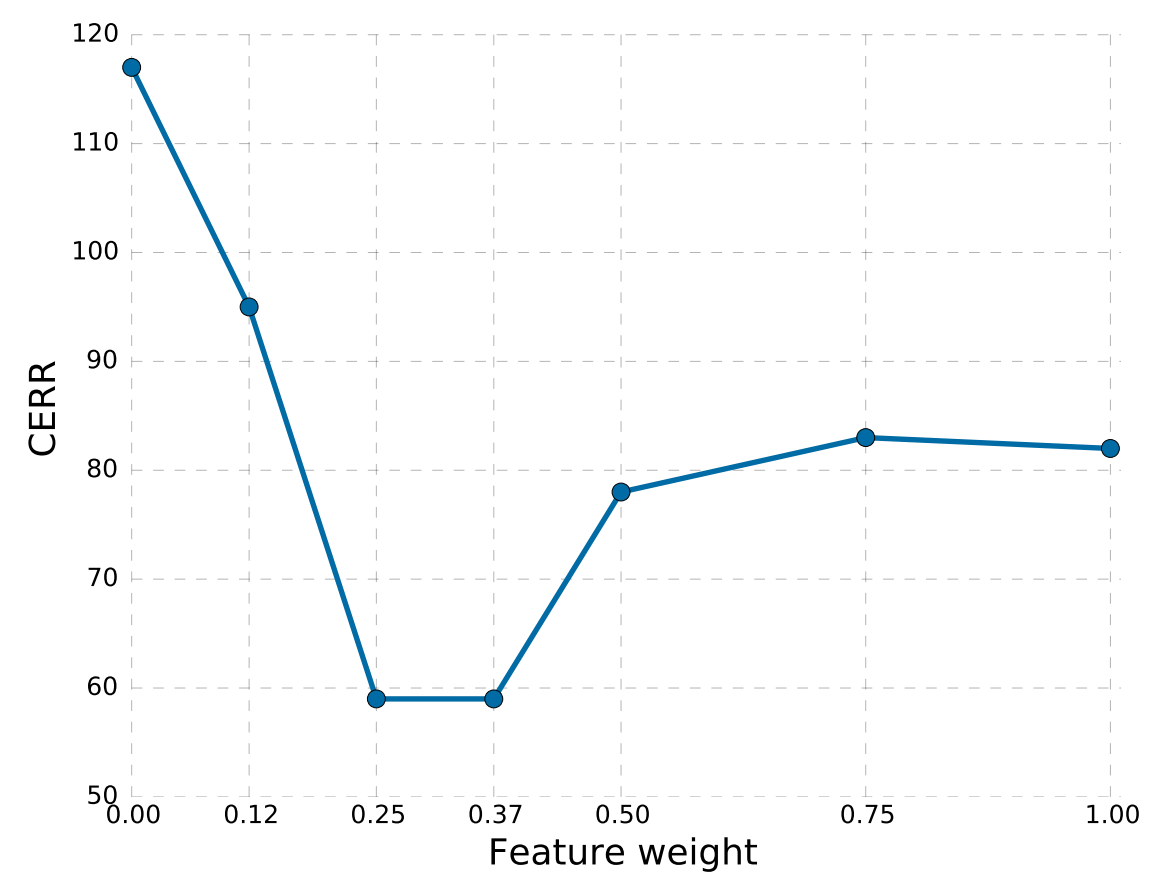

Figure 5.3: Center error according to feature weight $\rho_{F}$.

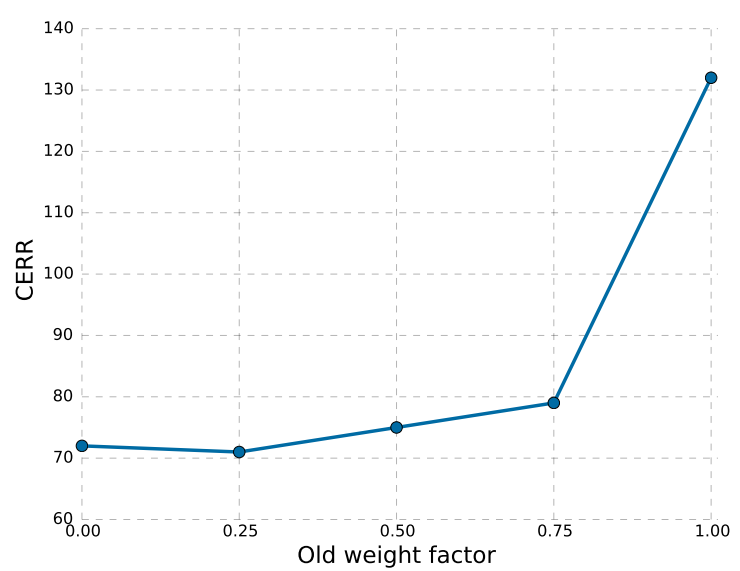

(a)

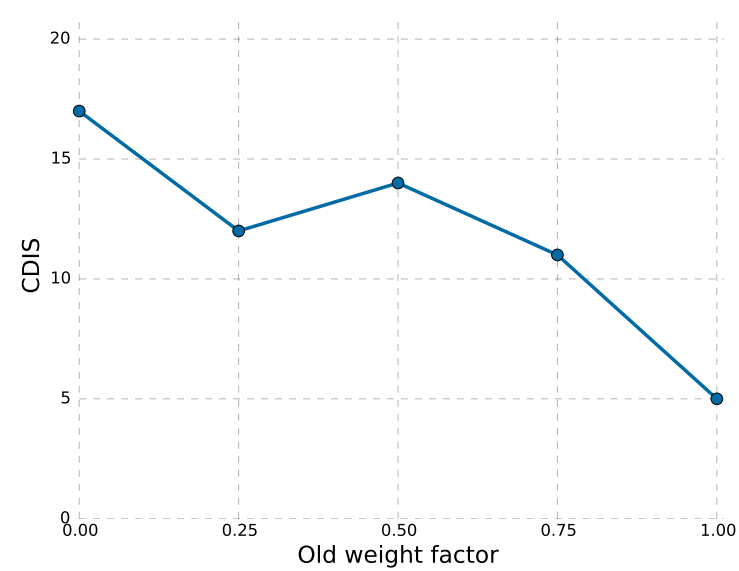

(b)

Figure 5.4: (a) Center error according to old weight factor $\rho_{T}$. (b) Variation in center stability caused by changing the parameter.

Table 5.2 summarizes all the parameters chosen for evaluation. The same values were used for all the experiments, independently of the dataset.

\subsubsection{Results on datasets}

We tested our approach on videos of table tennis doubles matches obtained from Youtube. These videos are interesting because they present challenging real world conditions like appearance change, occlusion and camera cuts. They also present some structure enforced by the rules of the game, which is captured by the graph model. 


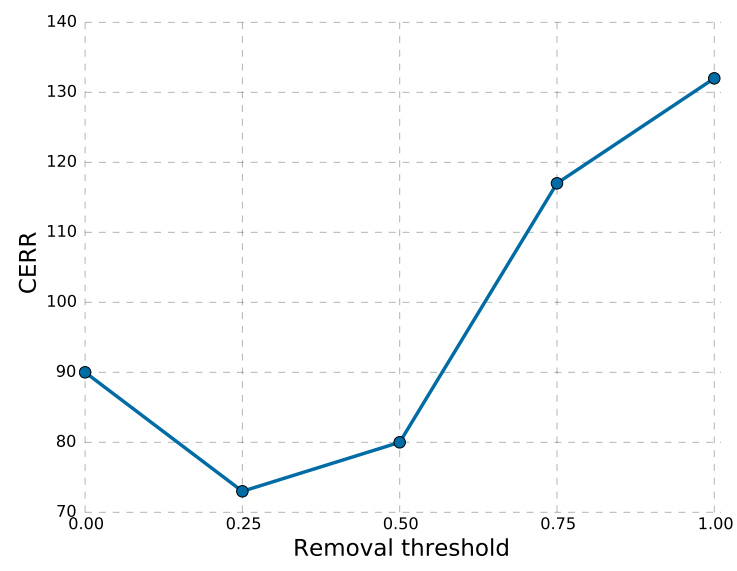

(a)

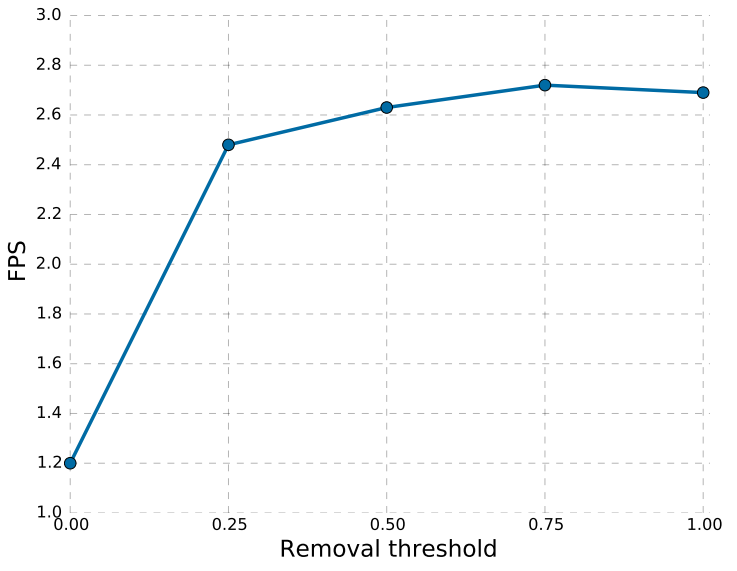

(b)

Figure 5.5: (a) Center error according to threshold for removing trackers $\tau_{S}$. (b) Variation in performance (frames per second) caused by changing the parameter.

Table 5.2: Parameters for the tracking framework.

\begin{tabular}{ll}
\hline number of particles per object & $N_{p}=50$ \\
initial particle spread deviation & $\sigma_{c}=10$ \\
overlapping distance between same object & $\tau_{d_{S}}=50$ \\
overlapping distance between different objects & $\tau_{d_{D}}=25$ \\
feature weight & $\rho_{F}=0.4$ \\
old temporal weight factor & $\rho_{T}=0.75$ \\
score threshold for removing trackers & $\tau_{S}=0.25$ \\
threshold for changing tracker & $\tau_{T}=1.25$ \\
threshold for changing after overlapping & $\tau_{O}=0.75$ \\
\hline
\end{tabular}

The task in these videos was to track all the four players and the table. We purposely track using only the torso of the players in order to create more appearance ambiguity and check whether the graph model can deal with this situation. As before, all the tests were performed five times and the average of all of the results was taken. The model graph was learned using a leave-one-video-out approach. The candidates matrix is the same as in the synthetic tests, while the chosen adjacency matrix was:

$$
M_{A}=\left(\begin{array}{lllll}
0 & 1 & 0 & 0 & 1 \\
1 & 0 & 0 & 0 & 1 \\
0 & 0 & 0 & 1 & 1 \\
0 & 0 & 1 & 0 & 1 \\
1 & 1 & 1 & 1 & 0
\end{array}\right) .
$$

This matrix considers the relations between players of the same team and all the players and the table. The relationship with the table is important because the players should be close and on opposite sides of it during the game. On the other hand, exploring the relationships 
between players of the same team helps to deal with temporary occlusions.

We compare our approach with other methods from the literature. The first one was the same particle filter color tracker we used, but without the graph information. In this way we could verify whether the addition of graphs bring any significant improvement to the classical approach. The second one was SPOT (Zhang and van der Maaten (2014)), that was mentioned in Chapter 2. As explained before, this tracker also considers structural information and, therefore, it is a good baseline for our results. The last tracker is STRUCK (Hare et al. (2011)), a single object tracker that, according to a recent benchmark (Wu et al. (2015)) was the best performing method in several datasets.

The results are presented in Table 5.3. The values correspond to the average of the results obtained from all five videos weighted by their respective number of frames. As pointed by all the measurements, the use of graphs clearly improves the tracking results. Particularly, the use of graphs successfully improves the results over the regular particle filter tracker. One point to note is that STRUCK performed similarly or worse than the particle filter approach. That is explained because the videos in the dataset often contain many situations of camera cuts. When this happens, both PF and STRUCK can only recover tracking when the target gets close to the point where it was lost. In that sense, the particle filters usually are able to recover the target more often because the particles usually are spread in a broader area than the STRUCK search radius. Since STRUCK conducts a dense neighbor search, as opposed to the sampled spread of PF, its search area must be kept smaller and thus, it is unable to redetect the target in many situations. It can also be observed that SPOT did not provide good results on these datasets. According to the observed results, the main reason seems to be that the structural model used by SPOT is sometimes too rigid and not very suited for a situation where the structural properties between the objects are subject to large changes in a short amount of time, such as in these sports videos.

Table 5.3: Observed results on both datasets. The arrows indicate whether lower or higher values are better.

\begin{tabular}{l|l|c|c|c|c}
\hline Dataset & Method & CERR $\downarrow$ & OBJI $\uparrow$ & HITR $\uparrow$ & HITT $\uparrow$ \\
\hline Youtube table tennis & PF + Graph & $\mathbf{4 2}$ & $\mathbf{0 . 5 6}$ & $\mathbf{0 . 7 1}$ & $\mathbf{0 . 7 9}$ \\
& PF & 103 & 0.45 & 0.56 & 0.61 \\
& SPOT & 81 & 0.34 & 0.40 & 0.44 \\
& STRUCK & 155 & 0.37 & 0.48 & 0.49 \\
\hline ACASVA badminton & PF + Graph & $\mathbf{4 0}$ & $\mathbf{0 . 5 0}$ & $\mathbf{0 . 6 3}$ & $\mathbf{0 . 8 1}$ \\
& PF & 67 & 0.41 & 0.51 & 0.60 \\
& SPOT & 59 & 0.27 & 0.32 & 0.37 \\
& STRUCK & 74 & 0.41 & 0.53 & 0.62 \\
\hline
\end{tabular}

Figure 5.6 shows some results observed on the videos. As it can be seen, our approach successfully recovers tracking after occlusion of camera cuts, while PF and STRUCK are not able to redetect the target after such situations. It is interesting to note that sometimes 
even the more robust STRUCK tracker is not able to deal with temporary occlusion, losing one of the targets, as shown in the third row of Figure 5.6. SPOT, on the other hand, does not suffer significantly from the abrupt motion. However, as it is evident from the pictures, sometimes the more rigid model ends up causing many tracking misses at the same time. These results further evidence the flexibility of the proposed method, that is able to accept a wide range of spatial configurations.
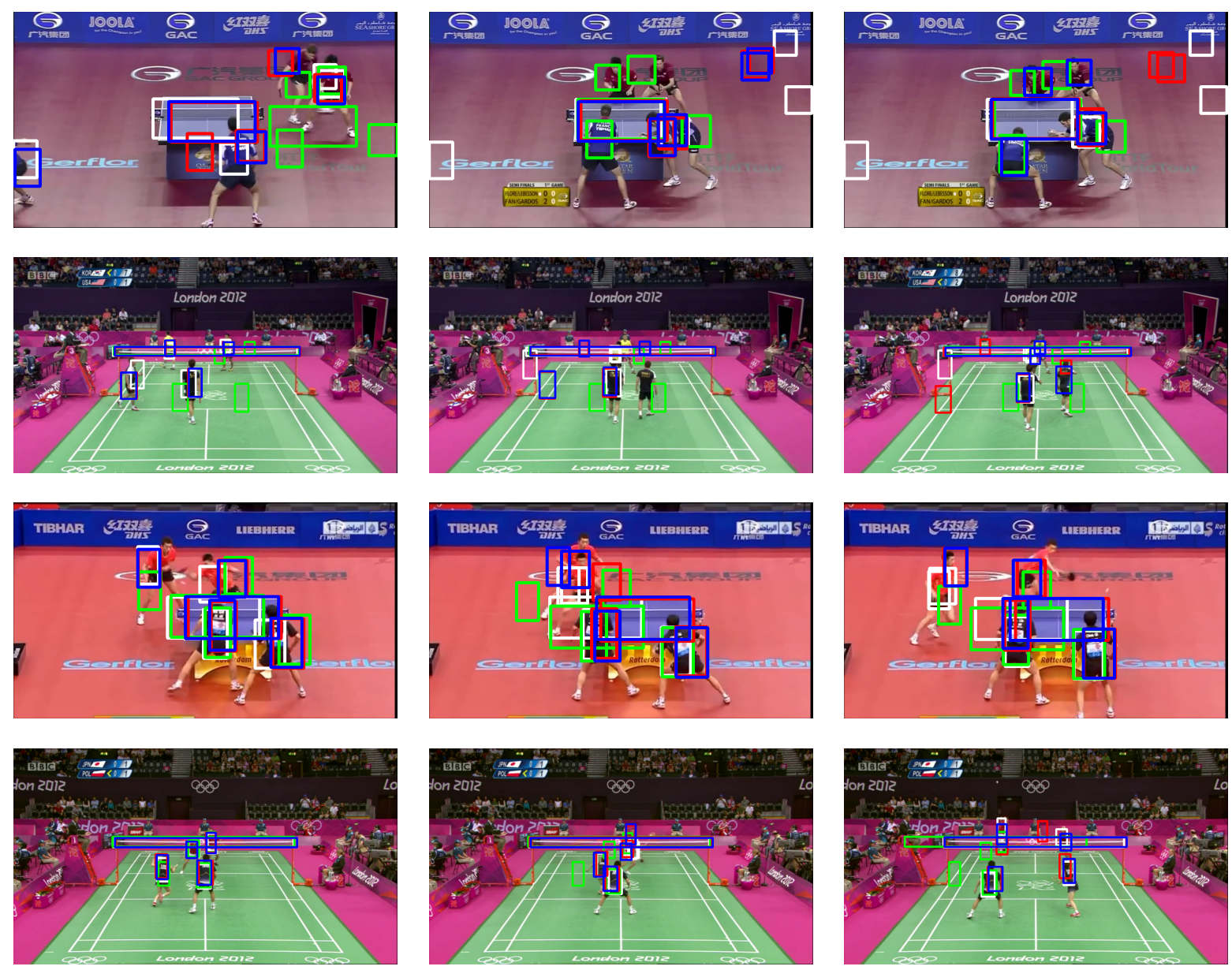

Figure 5.6: Tracking results for both datasets. Each method is represented by a different color. Blue: ours, red: particle filter, green: SPOT, white: STRUCK. The first two rows show results after a camera cut, while the last ones show situations of momentary occlusion. The first column represents the initial situation, in the second column, camera cut or occlusion happens and then, the third one shows the results after a few frames.

We also evaluated the behavior of each tracker during the video. Figure 5.7 shows how the CERR and HITT vary over time in a video. As it is more clear on the charts from the ACASVA dataset, both PF and STRUCK suffer from the drift problem over time. Although they are good at the beginning, once they lose the target, it is difficult to recover, thus causing larger errors as the videos move on. The analysis of the Youtube video also shows that the STRUCK tracker did not perform well on this video. This is explained because the chosen video contains many camera cut situations. As mentioned before, this is a particularly 
challenging situation for trackers based on local search only. In that sense, it can be seems that the use of structural information is successful in keeping the tracking more reliable even during longer videos.

\section{Youtube table tennis}
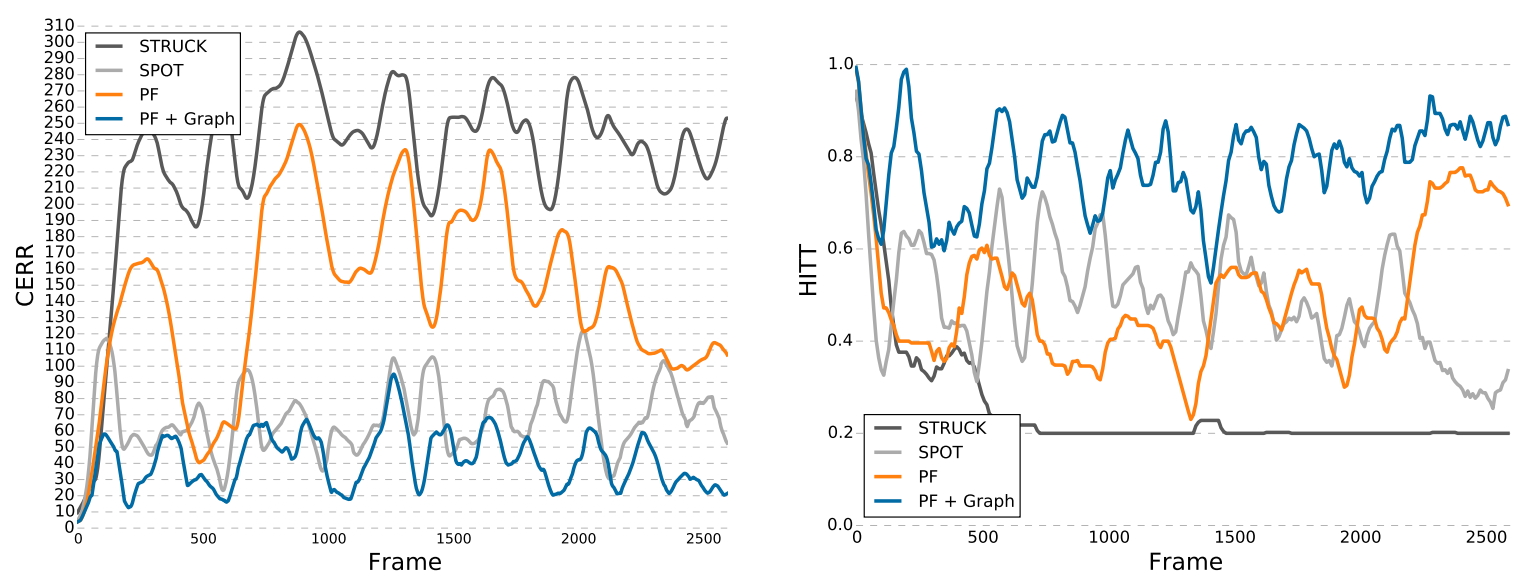

ACASVA badminton
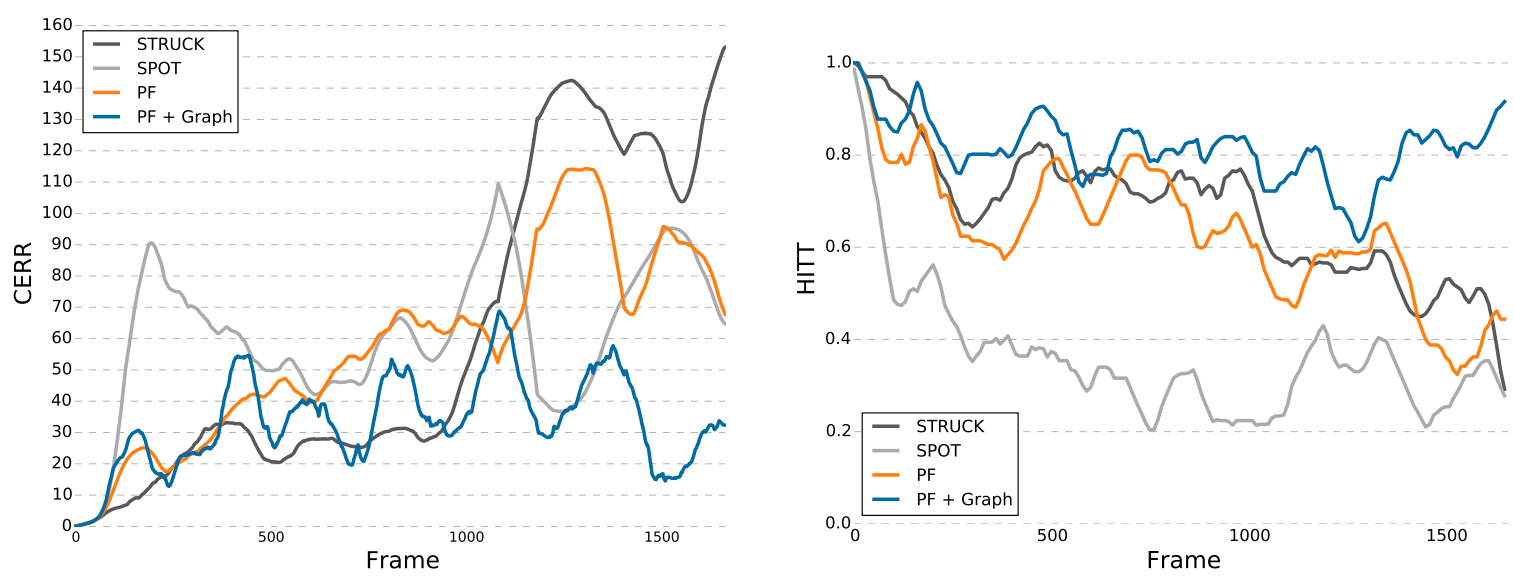

Figure 5.7: Center error and hit team ratio for one video from each dataset including object overlapping and camera cuts. The charts were smoothed using a moving average window of 100 frames.

\subsection{Action recognition}

The action recognition module was inspired by the work of $\mathrm{Lu}$ and Little (2006) and was implemented using PCA-HOG descriptors classified by HMMs. The HOG descriptors are extracted using OpenCV, while the PCA and HMM are implemented by the sklearn library $^{2}$.

The HOG descriptors are extracted from the bounding boxes of the objects obtained from the tracking module. However, different from Lu and Little (2006), HOGs are extracted as

\footnotetext{
${ }^{2}$ http://scikit-learn.org/stable/
} 
proposed by the original authors (Dalal and Triggs (2005)). As the observed results using this approach proved to be reasonable, we decided to keep the current implementation. In fact, this should not have a strong impact on the results, as the descriptor proposed by $\mathrm{Lu}$ and Little (2006) is just a simpler version of the original.

\subsubsection{Dataset}

The action recognition module was tested using videos of table tennis matches obtained from Youtube. As opposed to the ones using for testing the tracking module, the videos in this dataset were obtained from singles matches (one player at each side of the table). The main reason for this choice was to obtain a clearer view of each player as they performed their actions, decreasing the possibility of occlusion. The dataset was composed of 15 videos containing 9427 frames. All the frames were manually annotated with bounding boxes for each player as well as action labels. Figure 5.8 shows a few example images collected from the dataset used in this test.
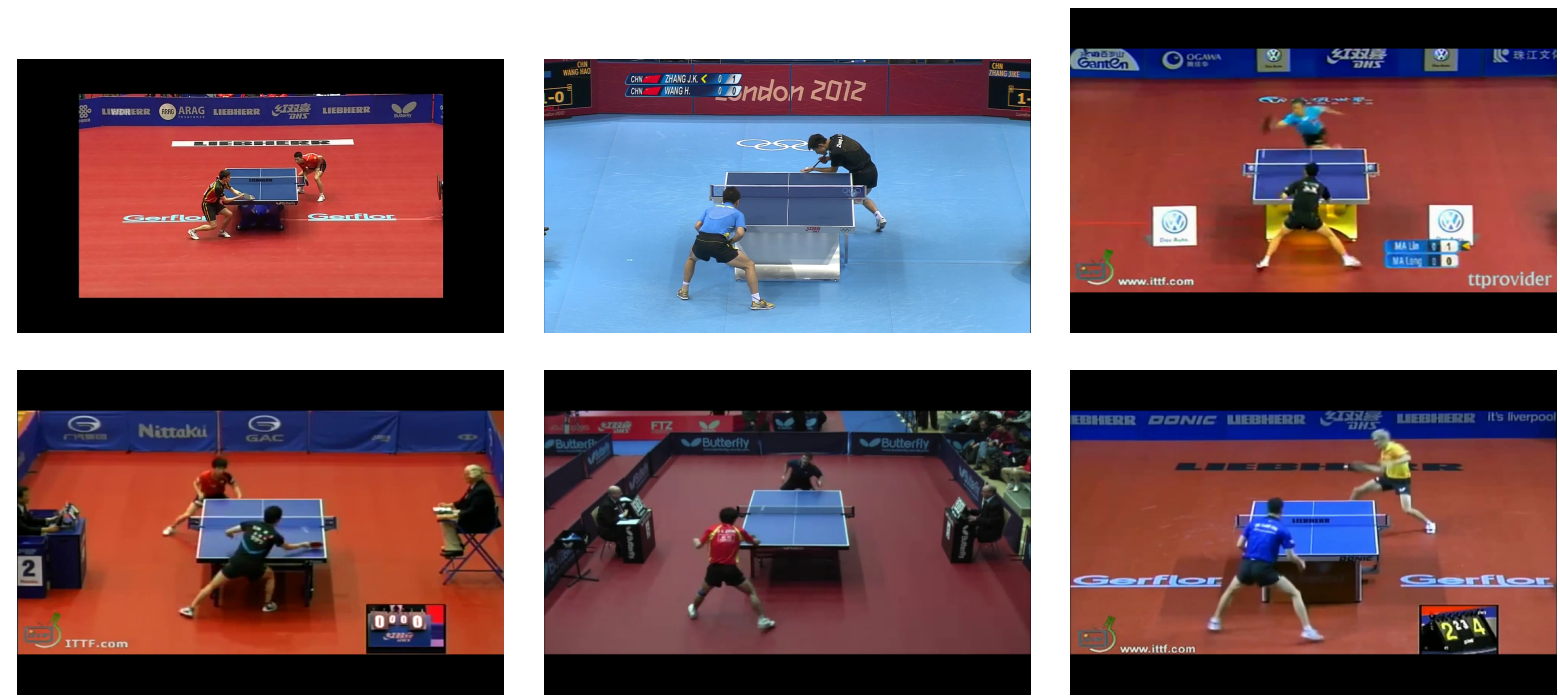

Figure 5.8: Sample frames from the dataset used for action recognition.

Based on the work of Wang et al. (2013), we decided to recognize four actions performed by each player: serve, backhand, forehand and others, where the last one is merely a wildcard that represents anything else. As the background is very different for each player (e.g. the player in the back is partially occluded by the table), a different set of HMMs was trained for each one, yielding a total of 8 HMMs.

The videos were separated into two sets, one for estimating the parameters of the descriptors and classifiers and another for evaluation. The estimation set was composed of 11 videos with 5672 frames, while the remaining 4 videos with 3755 frames were used for testing. The sets were divided in this way because the videos in the first set were shorter or 
with missing annotations. Therefore, we preferred to conduct the evaluation tests using the more complete videos.

\subsubsection{Estimation of parameters}

The parameters were estimated by following a 5-fold cross-validation approach on the estimation set and computing the average hit ratio for each configuration. The experiments involved tests for finding the best number of components from PCA, the length of one action sequence and the number of states of the HMMs.

Figure 5.9 shows the performance results for different number of principal components (PCs). The values correspond to the average between the two players. As it can be seen, the hit ratio reaches the top between 25 and 150 PCs. This result shows that, although a higher number of PCs encode more of the original information, it also hinders classification, as the search space increases exponentially. Due to the higher accuracy and considering the computer complexity, we chose to work with 25 PCs.

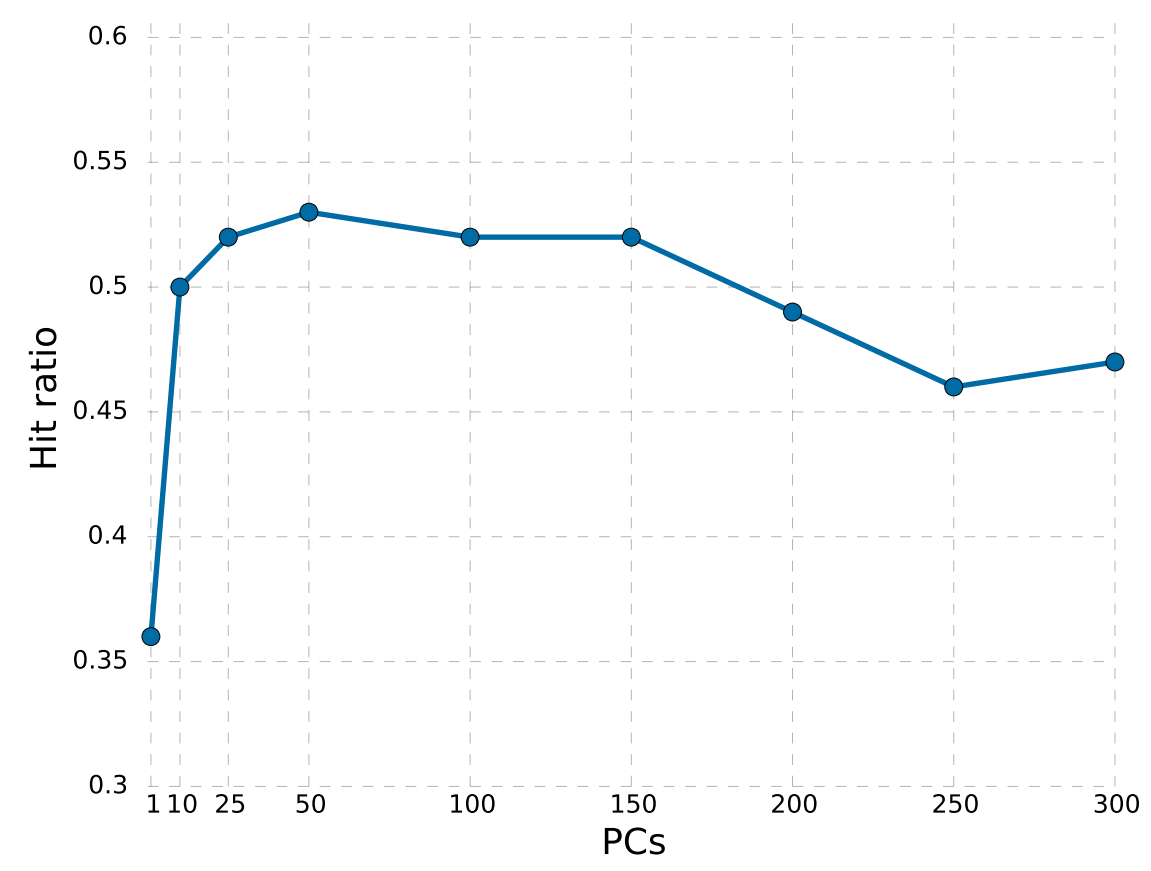

Figure 5.9: Variation of classification performance according to the numbers of PCs.

The sequence length and number of HMM states were tested simultaneously in order to find the best combination. The results are shown in Table 5.4. Results in the upper right part of the matrix are not shown due to a limitation in sklearn library that does not allow to train models with more states than the number of available observations. The table shows that changing these parameters does not affect significantly the performance. We have also tried to use different values for each object, but the observed results did not show any expressive changes. Since the results are very similar, we chose to work with the lowest parameters 
for both variables. The final parameters chosen for the evaluation task are summarized in Table 5.5.

Table 5.4: Classification hit ratio according to the sequence length and the number of hidden states.

\begin{tabular}{l|ccccc} 
seq.length / hmm states & 5 & 10 & 15 & 20 & 25 \\
\hline 5 & 0.54 & - & - & - & - \\
10 & 0.52 & 0.51 & - & - & - \\
15 & 0.55 & 0.56 & 0.43 & - & - \\
20 & 0.56 & 0.48 & 0.49 & 0.50 & - \\
25 & 0.54 & 0.53 & 0.54 & 0.49 & 0.42
\end{tabular}

Table 5.5: Final parameters for the action recognition.

\begin{tabular}{ll}
\hline number of principal components & 25 \\
sequence length & 5 \\
number of HMM states & 5 \\
\hline
\end{tabular}

\subsubsection{Results on dataset}

For this task, the bounding boxes obtained from tracking were collected beforehand and stored. Afterwards, they were segmented into individual action sequences according to the groundtruth. Therefore, the evaluation set was represented by several sequences, where each one should correspond to a complete action. The tests were then conducted in the same fashion as in the estimation phase. They were evaluated in a 5 -fold cross-validation approach where $80 \%$ of the sequences were used for training using the groundtruth data, while the remaining $20 \%$ sequences were provided by the tracking results.

We shall refer to as player 1, the player who is closer to the camera and thus, not occluded by the table, while player 2 is the other one. The results obtained for both players are shown in Table 5.6. As it is apparent, the results for player 2 are slightly worse than for player 1. This can be explained mainly due to the fact that this player may or may not be partially occluded by the table. Therefore, the shape descriptor may be different depending on the position of this player, which causes some classification issues. More detailed results can be seen in the confusion matrix presented in Table 5.7. Figure 5.10 shows some examples of action sequences that were classified.

Table 5.6: Observed hit ratio results for the action recognition task.

\begin{tabular}{ccc} 
& Mean & St. deviation \\
\hline Player 1 & 0.55 & 0.05 \\
Player 2 & 0.47 & 0.05 \\
\hline
\end{tabular}

The results show that the classification is reasonable for player 1 , while the player 2 presents more errors. The backhand action for player 1 presents the worse results, but this is 
Table 5.7: Confusion matrix of the action classification.

\begin{tabular}{ll|cccc} 
& & Others & Forehand & Backhand & Serve \\
\cline { 2 - 6 } Player 1 1 & Others & $\mathbf{0 . 7 2}$ & 0.12 & 0.16 & 0 \\
& Forehand & 0.28 & $\mathbf{0 . 5 2}$ & 0.20 & 0 \\
& Backhand & 0.44 & 0.16 & $\mathbf{0 . 4 0}$ & 0 \\
& Serve & 0.10 & 0.20 & 0.10 & $\mathbf{0 . 6 0}$ \\
& & Others & Forehand & Backhand & Serve \\
\cline { 2 - 6 } Player 2 2 Others & $\mathbf{0 . 6 8}$ & 0.12 & 0.20 & 0 \\
& Forehand & 0.48 & $\mathbf{0 . 2 0}$ & 0.32 & 0 \\
& Backhand & 0.40 & 0.08 & $\mathbf{0 . 5 2}$ & 0 \\
& Serve & 0 & 0.20 & 0.40 & $\mathbf{0 . 4 0}$
\end{tabular}
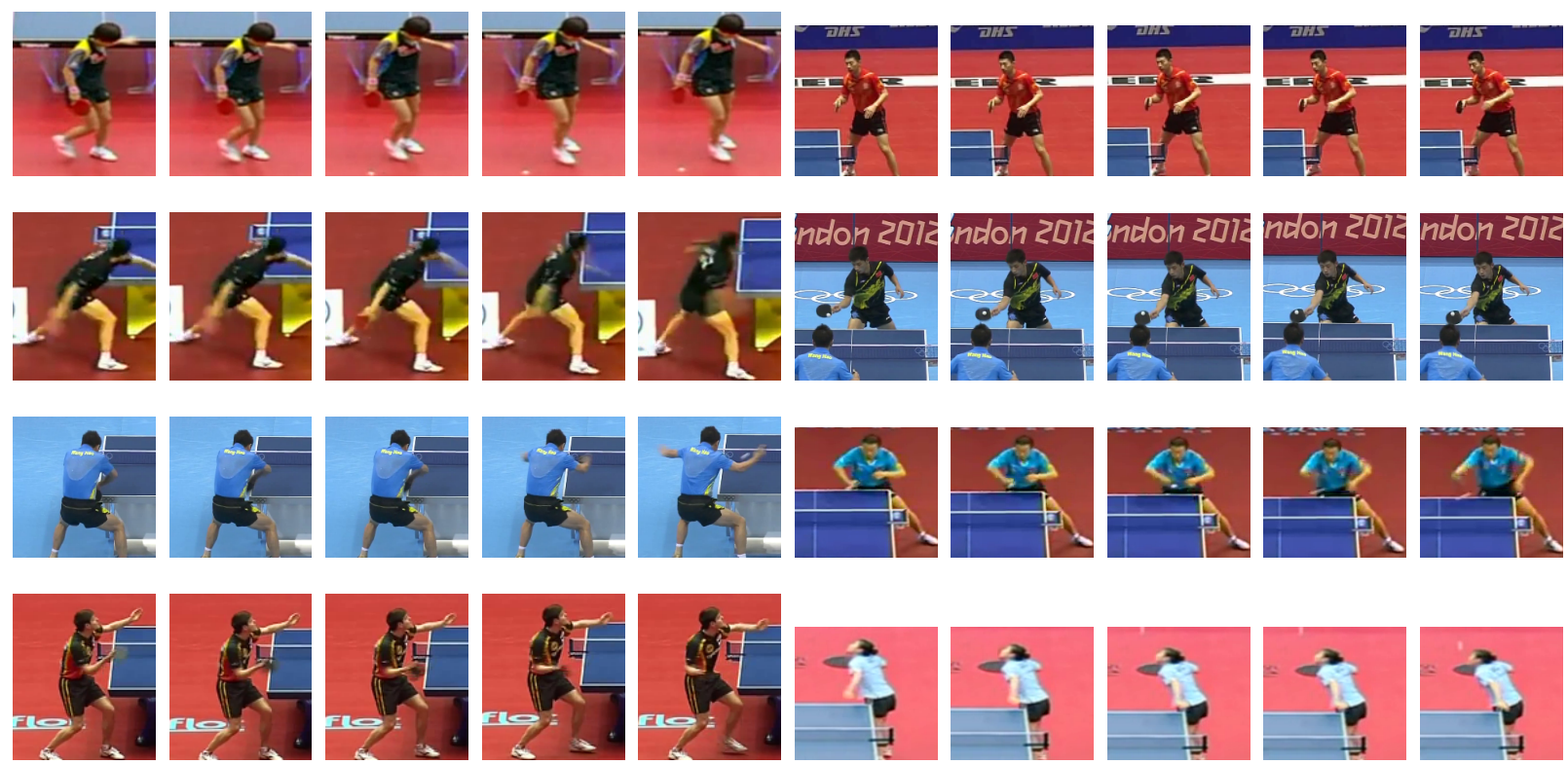

Figure 5.10: Examples of action sequences, the first five columns represent the action for player 1, while the others for player 2. Each row correspond to one action: (1) others, (2) forehand, (3) backhand and (4) serve.

not surprising. The reason is that, since the player has his back to the camera, this action is difficult to be perceived. The same can be said for player 2, on the opposite sense, where this action performs better, since his arms and hands are visible during this motion. However, it is clear that the classification for this player suffers for the reason explained before. 


\section{Chapter 6}

\section{Conclusions}

\subsection{Contributions and discussion}

We proposed a graph based approach to explore the structural information of a filmed scene and use it to improve tracking of multiple objects in structured videos. Each object in the scene represents one vertex of the graph, and edges are included to consider their spatial relations. Before the actual tracking, a probabilistic graph model is trained to learn the structure of the scene. The graph is then used for two purposes: (1) evaluate the current tracking state to check if the multiple objects are being correctly tracked and (2) generate new likely target locations to try to recover tracking in case it is lost. During the tracking, each object is individually tracked using particle filters. By merging the current tracking with the candidates generated by the model, multiple graphs are built. They are then evaluated according to the model, and the best one is chosen as the new global tracking state.

One of the advantages of the proposed framework is that, although during this thesis it was built over particle filters, it does not really rely on any information specific from that model. Therefore, the single object tracker could be potentially replaced by any other more suitable choice for other types of objects. This makes the method very flexible and able to deal with a wider range of applications.

We also used the tracking results to recognize the actions of the players in table tennis matches. This step was done by extracting PCA-HOG features from the image and then classifying them using HMMs. One different HMM was trained for each of the chosen actions (serve, backhand, forehand, others) using annotated data.

The results show that the proposed method successfully increases the tracking precision over other baseline methods on the sports datasets used in this thesis. As shown by the results this is mainly due to the fact that the use of the graphs allows us to recover tracking after challenging conditions such as temporary occlusion and camera cuts. The action recognition also showed convincing results, indicating it may be a viable option for further studying.

Therefore, based on the all the evidence collected during this thesis, we draw the following 
conclusions:

- When structural patterns can be found in the scene, the structural information plays an important role in improving multi-object tracking. As shown by the results, the combination of graphs with particle filter significantly improved the tracking results on the structured sports dataset over other approaches.

- A structural model complements the appearance descriptors, as the tracking precision is affected positively as structure information is combined with the visual features. However, appearance cannot be completely replaced by structure alone, as ignoring the former completely ends up decreasing the tracking performance.

- The drift problem can be greatly amended by relying on structural cues. As mentioned before, the drift is a serious challenge when tracking in longer videos. However, the collected results show that the proposed structural model successfully redetects lost targets after a short period of time, thus decreasing this problem.

\subsection{Limitations and Future work}

One limitation of the proposed framework is that the color-based tracker used for each object is not very robust against appearance or illumination changes. It is also sensible to initialization parameters, i.e. tracking may present poor results if the provided bounding box does not cover the object properly. As the graphs also use the tracker score for evaluating, if the color model is not representative enough, the whole tracking may be affected. This effect can be seen in the resulting videos, where sometimes objects with similar appearance change trackers, which might not happen if the proposed approach of generating new candidates is not used. However, as evidenced by the results, the overall result for all videos is better than when using other methods.

There are two main paths that we are interested in pursuing in the future to improve this method. The first one consists in making the method more self adaptive. One way to do so is to automatically adjust the number of candidates generated from each reference object, or to use the global structure as a whole to choose the best locations. This could be done by computing a reliability score for each object, combining the hypothesis of all of references into a single set and choosing only the best options.

Another option is, when working with particle filters, to automatically adjust the amount of particles generated for each candidate. This could improve the performance by generating fewer particles in locations that are not very likely to be correct. One direct approach for this is to use the graph score as a base for computing the number of particles. However, this method puts a lot of importance on the structure, and thus it may provide bad results if the 
trained model is not rich enough. One alternative would be to generate just small clouds of particles at each location and increase them in case the initial evaluation seems good.

Finally, the tracking can be extended by removing the need of training a model beforehand. An interesting research direction would be to adapt the method to train an adaptive model that could be improved as the tracking is happening.

The other path for improving this work concerns the action recognition module. In fact, we originally intended to use the action recognition results to feed the tracking module as well, creating a joint framework where both modules would contribute to the other. But, due to time constraints, this could not be implemented within this thesis. Nonetheless, this remains as a very interesting path to investigate in the future.

The main motivation for this approach is that one of the challenges of tracking is to correctly estimate the size of the bounding box surrounding the object. It is usually assumed that the proportions of the object do not change, but as evidenced by the players in the table tennis matches, it is easy to see that this assumption is not always reasonable. By knowing the action of a player, it would be possible to know whether the width of the bounding box needs to be increased (e.g. if the player spreads his arm) or reduced, for example.

\subsection{Scientific production}

The work developed during this thesis led the Ph.D. candidate to publish three papers in international conferences:

- Henrique Morimitsu, Roberto M Cesar Jr and Isabelle Bloch. Attributed graphs for tracking multiple objects in structured sports videos. In CVsports workshop of the International Conference on Computer Vision (ICCV). 2015.

- Henrique Morimitsu, Roberto M Cesar Jr and Isabelle Bloch. A spatio-temporal approach for multiple object detection in videos using graphs and probability maps. In Image Analysis and Recognition, pages 421-428. Springer. 2014.

- Henrique Morimitsu, Roberto M Cesar Jr and Isabelle Bloch. A graph-based approach for object detection and action recognition in videos. In FEAST Workshop of the International Conference on Pattern Recognition (ICPR). IAPR. 2014. 
64 CONCLUSIONS 


\section{Bibliography}

Adam et al. (2006) Amit Adam, Ehud Rivlin and Ilan Shimshoni. Robust fragmentsbased tracking using the integral histogram. In IEEE Conference on Computer Vision and Pattern Recognition, volume 1, pages 798-805. IEEE. Cited on page 49

Aggarwal and Ryoo (2011) Jake K Aggarwal and Michael S Ryoo. Human activity analysis: A review. ACM Computing Surveys (CSUR), 43(3):16. Cited on page 11

Blank et al. (2005) Moshe Blank, Lena Gorelick, Eli Shechtman, Michal Irani and Ronen Basri. Actions as space-time shapes. In IEEE International Conference on Computer Vision, volume 2, pages 1395-1402. Cited on page 11, 12

Blei et al. (2006) David M Blei, Michael I Jordan et al. Variational inference for dirichlet process mixtures. Bayesian analysis, 1(1):121-143. Cited on page 22

Bussab and Morettin (2010) Wilton de O Bussab and Pedro A Morettin. Estatistica básica. Saraiva. Cited on page 15

Callioli et al. (2007) Carlos Alberto Callioli, Hygino Hugueros Domingues and Roberto Celso Fabrício Costa. Álgebra linear e aplicações. Atual. Cited on page 34

Cho et al. (2013) Minsu Cho, Karteek Alahari and Jean Ponce. Learning graphs to match. In IEEE International Conference on Computer Vision, pages 25-32. Cited on page 21

Dalal and Triggs (2005) Navneet Dalal and Bill Triggs. Histograms of oriented gradients for human detection. In IEEE Conference on Computer Vision and Pattern Recognition, volume 1, pages 886-893. Cited on page xiii, 10, 22, 32, 56

De Campos et al. (2011) Teofilo De Campos, Mark Barnard, Krystian Mikolajczyk, Josef Kittler, Fei Yan, William Christmas and David Windridge. An evaluation of bags-ofwords and spatio-temporal shapes for action recognition. In Workshop on Applications of Computer Vision, pages 344-351. Cited on page 4, 47

Duda et al. (2001) Richard O Duda, Peter E Hart and David G Stork. Pattern classification. John Wiley \& Sons. Cited on page 34

Elgammal et al. (2000) Ahmed Elgammal, David Harwood and Larry Davis. Nonparametric model for background subtraction. In European Conference on Computer Vision, pages 751-767. Springer. Cited on page 21

Erdem et al. (2012) Erkut Erdem, Séverine Dubuisson and Isabelle Bloch. Fragments based tracking with adaptive cue integration. Computer Vision and Image Understanding, 116 (7):827-841. Cited on page 25 
Farnebäck (2003) Gunnar Farnebäck. Two-frame motion estimation based on polynomial expansion. In Image Analysis, pages 363-370. Springer. Cited on page 12

Felzenszwalb et al. (2010) Pedro F Felzenszwalb, Ross B Girshick, David McAllester and Deva Ramanan. Object detection with discriminatively trained part-based models. IEEE Transactions on Pattern Analysis and Machine Intelligence, 32(9):1627-1645. Cited on page 8,10

Figueroa et al. (2006) Pascual J Figueroa, Neucimar J Leite and Ricardo ML Barros. Background recovering in outdoor image sequences: An example of soccer players segmentation. Image and Vision Computing, 24(4):363-374. Cited on page 10

Fink (2008) Gernot A. Fink. Markov models for patter recognition: from theory to applications. Springer. Cited on page 34,40

Grabner et al. (2010) Helmut Grabner, Jiri Matas, Luc Van Gool and Philippe Cattin. Tracking the invisible: Learning where the object might be. In IEEE Conference on Computer Vision and Pattern Recognition, pages 1285-1292. Cited on page 8

Hare et al. (2011) Sam Hare, Amir Saffari and Philip HS Torr. Struck: Structured output tracking with kernels. In IEEE International Conference on Computer Vision, pages 263-270. Cited on page 53

Harris and Stephens (1988) Chris Harris and Mike Stephens. A combined corner and edge detector. In Alvey vision conference, volume 15, pages 50. Cited on page 8, 11

Hu et al. (2015) Weiming $\mathrm{Hu}$, Wei Li, Xiaoqin Zhang and Stephen Maybank. Single and multiple object tracking using a multi-feature joint sparse representation. IEEE Transactions on Pattern Analysis and Machine Intelligence, 37(4):816-833. Cited on page 8

Isard and Blake (1998) Michael Isard and Andrew Blake. CONDENSATION - conditional density propagation for visual tracking. International Journal of Computer Vision, 29(1): 5-28. Cited on page 18

Karavasilis et al. (2015) Vasileios Karavasilis, Christophoros Nikou and Aristidis Likas. Visual tracking using spatially weighted likelihood of gaussian mixtures. Computer Vision and Image Understanding, 140:43-57. Cited on page 21

Kristan et al. (2009) Matej Kristan, Janez Perš, Matej Perše and Stanislav Kovačič. Closed-world tracking of multiple interacting targets for indoor-sports applications. Computer Vision and Image Understanding, 113(5):598-611. Cited on page 10

Kwon and Lee (2008) Junseok Kwon and Kyoung Mu Lee. Tracking of abrupt motion using Wang-Landau Monte Carlo estimation. In European Conference on Computer Vision, pages 387-400. Springer. Cited on page 11

Laptev and Pérez (2007) Ivan Laptev and Patrick Pérez. Retrieving actions in movies. In IEEE International Conference on Computer Vision, pages 1-8. Cited on page 12

Liu et al. (2013) Jingchen Liu, Peter Carr, Robert T Collins and Yanxi Liu. Tracking sports players with context-conditioned motion models. In IEEE Conference on Computer Vision and Pattern Recognition, pages 1830-1837. Cited on page 11 
Lowe (2004) David G Lowe. Distinctive image features from scale-invariant keypoints. International Journal of Computer Vision, 60(2):91-110. Cited on page 22

Lu and Little (2006) Wei-Lwun Lu and James J Little. Simultaneous tracking and action recognition using the PCA-HOG descriptor. In Canadian Conference on Computer and Robot Vision, pages 6-6. Cited on page 12, 55, 56

Mei and Ling (2011) Xue Mei and Haibin Ling. Robust visual tracking and vehicle classification via sparse representation. IEEE Transactions on Pattern Analysis and Machine Intelligence, 33(11):2259-2272. Cited on page 8

Milan et al. (2014) Anton Milan, Stefan Roth and Kaspar Schindler. Continuous energy minimization for multitarget tracking. IEEE Transactions on Pattern Analysis and Machine Intelligence, 36(1):58-72. Cited on page 9

Morais et al. (2014) Erikson Morais, Anselmo Ferreira, Sergio Augusto Cunha, Ricardo ML Barros, Anderson Rocha and Siome Goldenstein. A multiple camera methodology for automatic localization and tracking of futsal players. Pattern Recognition Letters, 39: 21-30. Cited on page 10

Okuma et al. (2004) Kenji Okuma, Ali Taleghani, Nando De Freitas, James J Little and David G Lowe. A boosted particle filter: Multitarget detection and tracking. In European Conference on Computer Vision, pages 28-39. Springer. Cited on page 7, 10

Pérez et al. (2002) Patrick Pérez, Carine Hue, Jaco Vermaak and Michel Gangnet. Colorbased probabilistic tracking. In European Conference on Computer Vision, pages 661-675. Springer. Cited on page 19, 47

Reid (1979) Donald B Reid. An algorithm for tracking multiple targets. IEEE Transactions on Automatic Control, 24(6):843-854. Cited on page 7

Schüldt et al. (2004) Christian Schüldt, Ivan Laptev and Barbara Caputo. Recognizing human actions: a local SVM approach. In International Conference on Pattern Recognition, volume 3 , pages $32-36$. Cited on page 11

Shlens (2014) Jonathon Shlens. A tutorial on Principal Component Analysis. arXiv preprint arXiv:1404.1100. Cited on page 34

Su et al. (2014) Yingya Su, Qingjie Zhao, Liujun Zhao and Dongbing Gu. Abrupt motion tracking using a visual saliency embedded particle filter. Pattern Recognition, 47(5):18261834. Cited on page 11, 49

Tang et al. (2014) Siyu Tang, Mykhaylo Andriluka and Bernt Schiele. Detection and tracking of occluded people. International Journal of Computer Vision, 110(1):58-69. Cited on page 8

Viola and Jones (2004) Paul Viola and Michael J Jones. Robust real-time face detection. International Journal of Computer Vision, 57(2):137-154. Cited on page 10, 12

Wang and Schmid (2013) Heng Wang and Cordelia Schmid. Action recognition with improved trajectories. In IEEE International Conference on Computer Vision, pages 3551-3558. Cited on page 11, 12 
Wang et al. (2013) Zhenhua Wang, Qinfeng Shi, Chunhua Shen and Anton van den Hengel. Bilinear programming for human activity recognition with unknown MRF graphs. In IEEE Conference on Computer Vision and Pattern Recognition, pages 1690-1697. Cited on page 56

Wen et al. (2014) Longyin Wen, Wenbo Li, Junjie Yan, Zhen Lei, Dong Yi and Stan Z Li. Multiple target tracking based on undirected hierarchical relation hypergraph. In IEEE Conference on Computer Vision and Pattern Recognition, pages 1282-1289. Cited on page 9

Widynski et al. (2012) Nicolas Widynski, Séverine Dubuisson and Isabelle Bloch. Fuzzy spatial constraints and ranked partitioned sampling approach for multiple object tracking. Computer Vision and Image Understanding, 116(10):1076-1094. Cited on page 7

Wu et al. (2015) Yaowu Wu, Jungyoul Lim and Ming-Hsuan Yang. Object tracking benchmark. IEEE Transactions on Pattern Analysis and Machine Intelligence, 37(9):1834-1848. Cited on page 49,53

Xing et al. (2011) Junliang Xing, Haizhou Ai, Liwei Liu and Shihong Lao. Multiple player tracking in sports video: A dual-mode two-way bayesian inference approach with progressive observation modeling. IEEE Transactions on Image Processing, 20(6):16521667. Cited on page 10

Yan et al. (2014) Xu Yan, Ioannis A Kakadiaris and Shishir K Shah. Modeling local behavior for predicting social interactions towards human tracking. Pattern Recognition, 47(4):1626-1641. Cited on page 9

Yang et al. (2007) Jun Yang, Yu-Gang Jiang, Alexander G Hauptmann and Chong-Wah Ngo. Evaluating bag-of-visual-words representations in scene classification. In International Workshop on Multimedia Information Retrieval, pages 197-206. Cited on page 12

Zhang et al. (2012) Kaihua Zhang, Lei Zhang and Ming-Hsuan Yang. Real-time compressive tracking. In European Conference on Computer Vision, pages 864-877. Springer. Cited on page 11

Zhang and van der Maaten (2014) Lu Zhang and Laurens JP van der Maaten. Preserving structure in model-free tracking. IEEE Transactions on Pattern Analysis and Machine Intelligence, 36(4):756-769. Cited on page 10, 53

Zhang et al. (2015) Shun Zhang, Jinjun Wang, Zelun Wang, Yihong Gong and Yuehu Liu. Multi-target tracking by learning local-to-global trajectory models. Pattern Recognition, 48(2):580-590. Cited on page 9

Zhang et al. (2013) Tianzhu Zhang, Bernard Ghanem, Changsheng Xu and Narendra Ahuja. Object tracking by occlusion detection via structured sparse learning. In IEEE Conference on Computer Vision and Pattern Recognition Workshops, pages 1033-1040. Cited on page 11

Zhou and Lu (2010) Xiuzhuang Zhou and Yao Lu. Abrupt motion tracking via adaptive stochastic approximation Monte Carlo sampling. In IEEE Conference on Computer Vision and Pattern Recognition, pages 1847-1854. Cited on page 11 